NOVEL PATHOPHYSIOLOGICAL FACTORS AND DIAGNOSTIC MARKER IN ORGANIC AND FUNCTIONAL BOWEL DISORDERS

\author{
Ph.D. thesis
}

Orsolya Inczefi M.D. 


\section{NOVEL PATHOPHYSIOLOGICAL FACTORS AND DIAGNOSTIC MARKER IN ORGANIC AND FUNCTIONAL BOWEL DISORDERS}

Ph. D. Thesis

Orsolya Inczefi MD

Theoretical Medicine Doctoral School

Supervisor: Richárd Róka M.D, Ph. D.

First Department of Medicine, University of Szeged

Szeged, 2020. 


\section{LIST OF FULL PAPERS THE THESIS BASED UPON}

I. Inczefi O, Bacquié V, Olier-Pierre M, Rincel M, Ringot-Destrez B, Ellero-Simatos S, Eutamène H, Bétoulières C, Thomas J, Laine J, Gros L, Lévêque M, Leonard R, Harkat C, Robbe-Masselot C, Roka R, Mercier-Bonin M, Theodorou V, Darnaudéry M, Turner JR, Ferrier L.: Targeted intestinal tight junction hyperpermeability alters the microbiome, behavior, and visceromotor responses. Cell Mol Gastroenterol Hepatol. 2020 Mar 5 [Epub ahead of print]

II. Annaházi A, Molnár T, Farkas K, Rosztóczy A, Izbéki F, Gecse K, Inczefi O, Nagy F, Földesi I, Szücs M, Dabek M, Ferrier L, Theodorou V, Bueno L, Wittmann T, Róka R.: Fecal MMP-9: a new noninvasive differential diagnostic and activity marker in ulcerative colitis Inflamm Bowel Dis. 2013 Feb;19(2):316-20.

IF: $5.475, \mathrm{D} 1$

III. Annaházi A, Ábrahám S, Farkas K, Rosztóczy A,Inczefi O, Földesi I, Szücs M, Rutka M, Theodorou V, Eutamene H, Bueno L, Lázár G, Wittmann T, Molnár T, Róka R.: A pilot study on faecal MMP-9: a new noninvasive diagnostic marker of colorectal cancer Br J Cancer. 2016 Mar 29;114(7):787-92.

IF: 6.176, D1

Sum of impact factors (IF) for base references implicated in the thesis: 11,651

\section{LIST OF PUBLICATIONS NOT RELATED TO THE THESIS}

I. Bálint L, Tiszai A, Kozák G, Dóczi I, Szekeres V, Inczefi O, Ollé G, Helle K, Róka R, Rosztóczy A. Epidemiologic characteristics of Helicobacter pylori infection in southeast Hungary World J Gastroenterol. 2019 Nov 14;25(42):6365-6372.

$$
\mathrm{IF}^{2018}: \text { 3,411, Q1 }
$$

II. Annaházi A, Ferrier L, Bézirard V, Lévêque M, Eutamène H, Ait-Belgnaoui A, Coëffier M, Ducrotté P, Róka R, Inczefi O, Gecse K, Rosztóczy A, Molnár T, Ringel-Kulka T, Ringel 
Y, Piche T, Theodorou V, Wittmann T, Bueno L. Luminal cysteine-proteases degrade colonic tight junction structure and are responsible for abdominal pain in constipationpredominant IBS. Am J Gastroenterol. 2013 Aug;108(8):1322-31.

IF: 9.213, D1

Sum of IF for base references: $\mathbf{2 4 , 2 7 5}$ 


\section{TABLE OF CONTENTS}

1. INTRODUCTION 1

1.1. Animal studies 1

1.2. Human studies 2

2. AIMS 4

3. MATERIALS AND METHODS 5

3.1. Ethical Considerations 5

3.2 Animal studies 6

3.2.1. Animals 6

3.2.2 In vivo permeability analysis 6

$\begin{array}{ll}\text { 3.2.3 Ussing chamber analysis } & 6\end{array}$

3.2.4 Follow-up of animal growth $\quad 7$

$\begin{array}{lll}\text { 3.2.5 Gastrointestinal transit } & 7\end{array}$

3.2.6 Gut microbiota composition analysis $\quad 7$

3.2.7 Open field test $\quad 8$

3.2.8 Colorectal distension (CRD) 8

3.2.9 C-Fos analysis 9

3.2.10 Statistical analysis of animal data 9

3.3 Human studies 9

3.3.1. Patient selection for the observations of faecal MMP-9 levels in ulcerative colitis/IBS-D patients/healthy controls comparison (UC- study) 9

3.3.2 Patient selection for the faecal MMP-9 measurements in colon cancer $\begin{array}{ll}\text { (CRC study) } & 10\end{array}$

$\begin{array}{lll}\text { 3.3.3 Sample storage } & 10\end{array}$

3.3.4 Validation assay of faecal MMP-9 measurement 11

3.3.5 Measurement of faecal MMP-9 11

3.3.6 Measurement of faecal calprotectin 12

3.3.7 Statistical analysis in the human studies 12

4. RESULTS 13

4.1. Animal studies 13

4.1.1. In vivo and in vitro permeability measurements 13

4.1.2. Body weight gain and intestinal transit 13 
4.1.3. Microbiota analysis 13

4.1.4. Open field test 15

4.1.5. Colorectal distension 15

4.1.6. C-Fos analysis $\quad 15$

4.2. Human studies 18

4.2.1. Validation assay of faecal MMP-9 measurement 18

4.2.2. Faecal MMP-9 levels in control, IBS-D and UC patients’ samples 19

4.2.3. Correlation between faecal MMP-9 levels with disease activity in UC 20

4.2.4. Correlation of faecal MMP-9 concentration with faecal calprotectin levels in UC 21

4.2.5. Faecal MMP-9 levels in groups of non-adenoma/adenoma/CRC patients 22

4.2.6. Faecal MMP-9 levels according to CRC localisation and stage 25

$\begin{array}{lll}5 . & \text { DISCUSSION }\end{array}$

5.1. Animal studies $\quad 27$

5.2. Human studies 29

6. NEW RESULTS ESTABLISHED IN THE THESIS 34

6.1. Animal studies 34

6.2. Human studies 34

$\begin{array}{ll}\text { 7. SUMMARY } & 35\end{array}$

8. ABBREVIATIONS 38

9. ACKNOWLEDGEMENTS 40

10. REFERENCES 41

$\begin{array}{ll}\text { ANNEXES } & 46\end{array}$ 


\section{INTRODUCTION}

\subsection{Animal studies}

Irritable bowel syndrome (IBS) is a functional gastrointestinal disorder characterized by continuous or remittent abdominal pain, bloating and altered bowel habits without recognized underlying organic aetiology [1]. Nowadays, 100 years after the first description of functional gastrointestinal diseases, 5-20\% of patients seeking a gastroenterologist are diagnosed with IBS, causing considerably high medical and indirect costs [2]. Despite of its epidemiological significance, the pathophysiology of IBS is still not completely understood. The current classification of IBS is based on the Rome criteria [1] that only stratify patients according to their bowel habits as diarrhoea (IBS-D), or constipation (IBS-C) predominant, with mixed symptoms (IBS-M), and patients, who meet diagnostic criteria for IBS but their bowel habits cannot be accurately categorized in any of the above subtypes are labelled as unclassified IBS (IBS-U) group. The known elements of the pathomechanism are perturbation in the brain-gut axis causing altered pain sensation and modulation, misbalance in intestinal microbiota, moderate gut immune activation referred to as "low-grade inflammation", intestinal motor dysfunctions and elevated gut permeability [3]. In addition, a higher level of anxiety is often observed in these patients [4]. According to the high prevalence of the disease, a better understanding of the pathophysiology would help to focus on appropriate therapies.

In the present thesis, we focus on the role of the elevated gut permeability in the genesis of IBS symptoms. Intestinal barrier impairment has been associated with a variety of human diseases, e.g. inflammatory bowel disease (IBD), celiac disease, and IBS [5, 6]. Stressful life events, infection and dysbiosis can induce elevated gut permeability, which is associated with visceral hypersensitivity [7, 8], and pain severity in patients [9]. Animal models have shown that stress-induced elevation of paracellular permeability is linked to the decrease of the tight junction proteins expression (e.g. occludin, claudins). This is as a key factor in actinomyosin-driven epithelial cytoskeleton contraction, in which the phosphorylation of the myosin light chain by myosin light chain kinase (MLCK) plays the major role $[10,11]$. 
Every subtype of IBS is represented by visceral hypersensitivity. Several factors were found playing a role in this phenomenon: modulation of the central nervous response to the intestinal derived stimuli, hyperexcitability of the afferent innervation, or sensitization of the sensory endings in the gut wall can contribute to the pain response [12]. End-organ hypersensitivity may be a result of the low-grade immune activation in the intestine. Moreover, mast cells and their mediators, such as histamine, trypsin, and tryptase have been documented in higher levels in colonic and jejunal biopsy supernatants from IBS patients, and increased mast cell infiltration has also been found in IBS gut mucosa. Similar results were found using animal models, and mast cell stabilizers have proven efficacy in preventing visceral hyperalgesia in animals $[13,14]$. Transgenic mice with specific intestinal expression of a constitutively active form of MLCK (CAMLCKTg) have congenitally elevated gut permeability. Besides this increased gut permeability, these animals present a moderate intestinal immune activation in basal state without any sign of disease [15], which suggests that it could represent an ideal model to study IBS.

Nowadays it is well known, that alteration of the intestinal microbiota is present in IBS patients [16, 17], however the role of dysbiosis in the pathophysiology is unclear. Infective diarrhoea can provoke long-term IBS symptoms, and intestinal microbiota is permanently perturbed after the elimination of the pathogen (postinfectious IBS) [18]. On the other hand, modification of the intestinal microbiota (e.g. rifaximin or probiotic treatment) in IBS patients has only short term effect on patient's symptoms. [19]. The temporary efficacy of these treatments could suggest an endogenous trigger maintaining the "perturbed" bacterial composition in the intestine. CAMLCKTg animal model could help to clarify the potential relationship between the intestinal permeability changes and the composition of the intestinal microbiota.

\subsection{Human studies}

Ulcerative colitis (UC) is a chronic, relapsing inflammatory bowel disease characterized by continuous colonic mucosal ulceration and diarrhoea during relapses. The differentiation of UC from functional disorders such as IBS-D is clinically challenging, as 
symptoms may include altered bowel habits and abdominal pain in both diseases, morover activity signs can mix with anxiety-driven symptoms as well in UC. Several attempts have been made to establish non-invasive markers that can clearly distinguish IBD from functional disorders and reveal strong correlation not only with clinical activity, but also with the endoscopic picture of the mucosa in UC. Among biochemical laboratory markers serum Creactive protein (CRP) is the most studied [20]; however, while the activity of Crohn's disease (CD) is accompanied by a strong CRP response in most patients, relapses in UC are characterized by only a modest or absent CRP increase [21] depending mostly on the extension of the involved area. The sensitivity of CRP in the detection of UC is only $50 \%-$ 70\% [22-24]. The pathogenesis of UC includes the complex dysregulation of mucosal immune cells [25] accompanied by the invasion of neutrophils, which leads to the formation of crypt abscesses and to the dysfunction of the colonic epithelial barrier [26]. During transmigration, neutrophils reach the colonic lumen and can be detected in the stool [27], similarly to their secreted mediators, such as polymorphonuclear elastase, calprotectin, or cathepsin G [28, 29]. Therefore, faecal biomarkers that reflect neutrophil activation seem promising in UC, and faecal calprotectin has been identified as a marker to discriminate IBD patients from IBS patients or controls [29, 30], and its faecal level correlates with disease activity [31, 32]. Matrix metalloproteases (MMPs) 1, 2, 3, and 9 are also released from neutrophils in IBD, and they are significantly elevated in colonic biopsies from UC patients compared to controls, with a higher increase in the ulcerated than in nonulcerated regions [33]. Among these MMPs, MMP-9 was the most abundant in UC biopsies [33]. Additionally, elevated MMP-2 and MMP-9 levels were detected in the urine of paediatric patients suffering from either UC or CD, and these MMPs were suggested as non-invasive biomarkers in the diagnosis of IBD [34]. Nevertheless, no study has so far focused on faecal MMP levels in UC compared with IBS-D. Furthermore, the correlation of MMPs with disease severity, clinical, and endoscopic activity has never been evaluated.

Colorectal cancer (CRC) represents a major cause of morbidity and mortality in Western societies, and the incidence in developing countries is also rapidly growing [35-37]. The high frequency of the disease and the fact that the prognosis well correlates with the stage at diagnosis makes CRC screening extremely important. Colonoscopy is the gold standard for CRC screening by its high sensitivity and specificity but has also disadvantages, such as risk of complications, high costs and lower acceptance by patients as a screening method. 
Therefore, non-invasive methods are required to identify patients at high risk of CRC where colonoscopy needs to be performed. CRC screening recommendations vary between countries but mostly consist of an annual or biannual faecal occult blood test (FOBT) combined with colonoscopy in case of positivity or a colonoscopy every 10 years, in the population aged $>50$ years [38]. Two basic types of FOBT exist, based on the imperceptible intermittent leakage of blood from CRC and high-risk adenomas in stool [38]. The guaiac test (gFOBT) detects the peroxidase activity in the haemoglobin heme subunit, therefore it is nonspecific for human haemoglobin and theoretically requires dietary restrictions a few days before performing the test, but large studies have found no significant advantage of the dietary modifications, therefore lately they are not recommended by many authors [39]. A minimum of two faecal samples are necessary from different stools to obtain an appropriate sensitivity. Sensitivity for CRC ranges from $6.2 \%$ to $83.3 \%$, depending on the test used, while specificity is $>80 \%$ [38]. The immunochemical faecal occult blood test (FIT) is based on specific monoclonal or polyclonal antibodies against human haemoglobin, thus it does not require dietary alterations. Contrary to gFOTB, the analysis by FIT can be automated and its threshold value is adjustable by the user. According to the literature, the sensitivity of FIT is extremely variable, from $5.4 \%$ to nearly $98 \%$, depending on the test used [38]. Specificity can range from $77 \%$ to 99\%. The European Guidelines for quality assurance of CRC recommends the use of FIT rather than gFOBT [40]. However, reliable tests with a less variable sensitivity and specificity are still lacking. MMPs are a family of $\mathrm{Zn}^{2+}$ containing endopeptidases, secreted by various cell types, such as tumour cells, mesenchymal cells, fibroblasts and inflammatory cells (monocytes, lymphocytes and neutrophils). Increased expression of MMP-9 has been detected in tissue samples from rectal carcinoma and colon cancer, where it correlates with poor prognosis [41-43]. However, faecal MMP-9 levels in CRC patients have not been investigated.

\section{AIMS}

In animal experiments in CAMLCKTg mice our aims were:

1) to verify if the transgene has an effect on the gut permeability, animals' growth and intestinal transit; 
2) to explore the effect of elevated intestinal permeability on the gut microbiota composition;

3) to study the moderately elevated gut permeability on behaviour and visceral sensitivity;

4) to investigate if the intestinal permeability elevation has an effect in the brain activity.

In human studies our aims were:

1) to validate if the faecal matrix has an influence on MMP-9 determination and whether the kit is able to determine MMP-9 from human faecal extracts;

2) to compare faecal MMP-9 levels in UC patients to control subjects and patients with a functional gastrointestinal disorder characterized by diarrhoea (IBS-D);

3) to test the correlation between UC disease activity and faecal levels of MMP-9;

4) to correlate faecal MMP-9 levels with a known faecal marker of UC activity, calprotectin;

5) To compare faecal MMP-9 levels in patients with normal colonoscopic results, colonic polyps and colon cancer.

\section{MATERIALS AND METHODS}

\subsection{Ethical Considerations}

Experimental procedures on animals were reviewed and authorized by the Ethical Committee CEEA-86, under the number APAFiS\#4145. (INRA TOXALIM, Toulouse, France)

The human study protocols were approved by the Ethical Committee of the University of Szeged under the number 170/2011. All subjects provided written and informed consent to participate. 


\subsection{Animal studies}

\subsubsection{Animals}

CAMLCKTg mice (Tg(Vil-FLAG-CAMLCK)\#Jrt) were maintained as male heterozygotes on C57BL/6J background. These were mated with wild type (WT) C57BL/6J females to produce WT and CAMLCKTg littermates. At weaning, female mice were separated and housed at constant temperature $\left(22 \pm 1^{\circ} \mathrm{C}\right)$ with a 12 hour light/dark cycle. Food (Teklad 2018, Envigo) and water were available ad libitum. All experiments were performed at 8 weeks of age.

\subsubsection{In vivo permeability analysis}

Mice were fasted for 4 hours before gavage with $150 \mu \mathrm{L}$ of $100 \mathrm{mg} / \mathrm{mL}$ FITC-4kDa dextran in tap water. Blood $(200 \mu \mathrm{L})$ was collected after $4 \mathrm{~h}$ and plasma fluorescence determined using Tecan Infinite 200 microplate reader (Tecan Group Ltd., Switzerland).

\subsubsection{Ussing chamber analysis}

Animals were sacrified and jejunal sections were collected. Jejunal sections were mounted in Ussing chambers (Physiologic Instruments, USA) filled with Krebs buffer and continuously oxygenated (95\% O2, 5\% CO2). After 1 hour of equilibration, Fluorescein $(1 \mathrm{mg} / \mathrm{mL})$ was added in the apical chamber and fluorescence intensity of the basolateral chamber was measured after 1 hour using Tecan Infinite 200 microplate reader (Tecan Group Ltd., Switzerland). 


\subsubsection{Follow-up of animal growth}

CAMLCKTg and WT mice were weighed weekly for 12 weeks. (Cubis, Sartorius, Germany)

\subsubsection{Gastrointestinal transit}

Animals received $70 \mu \mathrm{L}$ of $100 \mathrm{mg} / \mathrm{ml}$ TRITC-70kDa dextran in tap water by gavage and were sacrificed $1 \mathrm{~h}$ later. Stomach, small and large intestine were cut in 11 equal parts. Luminal contents of each segment were centrifuged and fluorescence determined using Tecan Infinite 200 microplate reader (Tecan Group Ltd., Switzerland). Transit was calculated as the geometric center of the values for each mouse.

\subsubsection{Gut microbiota composition analysis}

Gut microbiota were analysed in two cohorts (15 WT and 16 CAMLCKTg) from 8 different WT dams. At sacrifice, colonic contents were stored at $-80^{\circ} \mathrm{C}$. DNA was extracted using the ZR faecal DNA MiniPrep kit (Zymo Research, USA) and adjusted to $1 \mathrm{ng} / \mu \mathrm{L}$. Changes in relative abundance of 24 microbial 16S rRNA gene targets were obtained by qRTPCR using an adapted Gut Low- Density Array platform [44-46]. A universal bacterial primer set was included as the reference gene. The qRTPCR was performed in duplicate on a ViiA7 (Applied Biosystems, USA). Fluorescence data was imported into LinRegPCR to perform baseline corrections, calculate mean PCR efficiency per amplicon group and calculate initial quantities. Among the 24 targeted amplicon groups, 6 were not detected in any faecal samples and were removed from the analysis (B. vulgatus, Alistipes spp., Parabacteroidetes distasonis, Roseburia spp., E. coli and A. muciniphila). Normalized N0values were log10-transformed and processed by MixOmics (v6.1.1) with RStudio (v1.0.44) to build a partial least-squares discriminant analysis (PLS-DA). This multivariate supervised 
approach projects samples $(\mathrm{X})$ onto a low-dimensional space of latent variables to maximize separation between groups according ( $\mathrm{Y}=$ =genotype). Leave-one-out cross-validation was used to select the optimal number of latent variables for PLS-DA models.

\subsubsection{Open field test}

Mice explored a 50x50 cm arena (illumination 300lux) for 10 min. Exploration was automatically assessed using a video tracking system (Bioseb, France). The percentage of distance travelled and time spent and in the center area $(20 \times 20 \mathrm{~cm})$ and total distance travelled in the entire arena were assessed.

\subsubsection{Colorectal distension (CRD)}

Two 0,08 $\mathrm{mm}$ diameter electrodes were implanted in the abdominal external oblique muscle and a third in the abdominal skin. On postoperative days 3-6, CRD was performed using a balloon catheter (Fogarty 4F catheter, 1,1 cm length, and tip 3,5 cm from the anus) in $10 \mathrm{sec}$ periods with increasing volumes from $0,02 \mathrm{~mL}$ to $0,10 \mathrm{~mL}$, with 5 min rest between distensions. Abdominal electromyography activity was registered after the amplification (10000x) and analysed (Powerlab Chart 5, AD Instruments, Australia). Basal EMG activity was subtracted from EMG activity registered during distension. Some mice were treated with ML-7 (2 mg/kg i.p.) or naloxone sulfate (2 mg/kg i.p.) $1 \mathrm{~h}$ before CRD. For others, water avoidance stress was induced on a floating platform $(3 \times 3 \mathrm{~cm})$ in the middle of a water-filled tank $(40 x 40 \mathrm{~cm})$ for $1 \mathrm{~h}$ daily over four days. Recovery (30 min) preceded CRD. 


\subsubsection{C-Fos analysis}

Vibratome sections $(40 \mu \mathrm{m})$ were stained using polyclonal rabbit anti-C-Fos (Santa Cruz Biotechnology, USA) and secondary HRP-conjugated goat anti-rabbit antisera (Jackson Immuno Research, UK). NDPI images (x20) were obtained (Nanozoomer, Hamamatsu Photonics, Japan) and converted into TIFF format using ImageJ (NDPI tools plugin, USA). Regions of interest (ROI) were manually circumscribed using ROI tools and C-Fosimmunoreactive cells quantified automatically using the particle analysis function (size: 5-20 $\mu \mathrm{m}^{2}$; circularity: 0,5-1). For each animal, 3-6 sections of each brain area were assessed by a blinded observer.

\subsubsection{Statistical analysis of animal data}

Statistical significance was determined by two-tailed t-test, two-tailed Mann-Whitney $\mathrm{U}$ test, or 2-way ANOVA and set at $p<0.05$. For microbial analyses, univariate analysis was realized in parallel to compare each amplicon separately using unpaired t-test followed by the Benjamini-Hochberg adjustment of $p$-values for multiple comparisons. For statistical analysis Graph Pad Prism 4.0 (GraphPad, San Diego, CA) was used.

\subsection{Human studies}

\subsubsection{Patient selection for the observations of faecal MMP-9 levels in ulcerative colitis/IBS-D patients/healthy controls comparison (UC- study)}

Overall, 94 patients were enrolled in the study. Healthy controls ( $n=24$; male/female: 9/15; mean age: 49.3 years), UC ( $n=47$; male/female: 19/28; mean age: 48.0 years), and IBS$\mathrm{D}$ ( $\mathrm{n}=23$; male/female: 4/19; mean age: 49.4 years) patients were recruited to provide faecal 
samples at the First Department of Medicine, Szeged, Hungary. IBS-D patients fulfilled the Rome III criteria. Organic gastrointestinal disorders were excluded by detailed blood analyses and stool cultures, serologic assays for celiac disease, lactose-hydrogen breath test, and colonoscopy. UC patients were evaluated clinically and endoscopically. The activity of the disease was determined by clinical (partial and total Mayo score) and endoscopic (Mayo endoscopic subscore) scores. Based on the total Mayo score, 12 patients were determined as inactive, 17 as mildly active, 9 as moderately active, and 9 as severely active. At the time of the endoscopy, blood samples were taken from all patients for the determination of platelet and leukocyte count, CRP, erythrocyte sedimentation rate, serum iron level, and haemoglobin level.

\subsubsection{Patient selection for the faecal MMP-9 measurements in colon cancer (CRC study)}

Overall, 109 patients (male/female: 58/51, age: 29-91 years, mean: 66.0 years) referred to the First Department of Medicine, Szeged, Hungary were enrolled in the study, who all underwent colonoscopy. No patients had a history of UC or a history of other cancers. One patient, who was later identified as a rectal adenocarcinoma patient, had liver metastases. All colonoscopies were completed until reaching the terminal ileum. During colonoscopy, biopsies were taken from all suspect lesions, and histology was performed. Based on the colonoscopic and histological results, patients were allocated to five groups: negative, diverticulosis, hyperplastic polyp, adenoma, and CRC. Adenomas were considered at increased risk of malignisation when one of the followings was fulfilled: polyp size $410 \mathrm{~mm}$; villous adenoma or tubulovillous adenoma with at least $20 \%$ villous component; high grade dysplasia; or multiple adenomas.

\subsubsection{Sample storage}

Faecal samples were collected from all patients 2-4 days before the colonoscopy and were frozen to $-20^{\circ} \mathrm{C}$ within 1 hour after defecation. 


\subsubsection{Validation assay of faecal MMP-9 measurement}

Our group was the first to use the commercial Human Quantitative Enzyme-Linked Immunosorbent Assay Kit from R\&D Systems (Abingdon, UK, Cat. No. DMP900) for the measurement of MMP-9 levels in faeces. Therefore, we have performed a validation assay to test whether the faecal matrix itself has an influence on MMP-9 determination and whether the kit from R\&D Systems is able to determine MMP-9 from human faecal extracts. Active human MMP-9 full-length protein (Abcam LTD, Cambridge, UK; Cat. No.: ab157344; LOT No: GR218764-1) was added to the extraction medium. Control faecal samples were weighed and $1 \mathrm{ml}$ extraction medium with or without added MMP-9 was given to $0.25 \mathrm{mg}$ sample (the sample to extraction medium ratio was 1:4). The samples were immediately homogenised for $2 \times 30 \mathrm{~s}$ and incubated for $10 \mathrm{~min}$ at room temperature. Subsequently, all homogenates were centrifuged at $1500 \mathrm{~g}$ for $10 \mathrm{~min}$ followed by a second centrifugation of the supernatants from the first centrifugation step at $10000 \mathrm{~g}$ for $10 \mathrm{~min}$. The final supernatants were processed for linearity (parallelism) test. The extract containing added MMP-9 was serially diluted $1+1$ to get 50, 25, 12,5 and 6,25\% of the original MMP-9 content with the extract of the same faeces containing no added MMP-9. Then all samples, including 100\% of added MMP-9 extract and the native control extract without added MMP-9, were subjected to MMP-9 measurement by the above-mentioned ELISA kit for human MMP-9. The kit is able to detect the human 92 kDa Pro-MMP-9 and 82 kDa active form of MMP-9 as stated by the manufacturer. The added MMP-9 protein obtained for this evaluation was the 92 kDa Pro-MMP-9.

\subsubsection{Measurement of faecal MMP-9}

Samples were stored on $-20{ }^{\circ} \mathrm{C}$ for a maximum of 3 months and then thawed at $4{ }^{\circ} \mathrm{C}$ for 1 day. In all, 1,0 g of each faecal sample was diluted, mixed and homogenised in $4 \mathrm{ml}$ of icecold Tris-buffer $(0,15 \mathrm{M} \mathrm{NaCl}+20 \mathrm{mM}$ Tris-HCl, $\mathrm{pH}: 8,3)$. After centrifugation (10 min, 4500 r.p.m., $4{ }^{\circ} \mathrm{C}$ ), pellets were discarded and supernatants were recentrifuged (10 min, 10000 g; 4 ${ }^{\circ} \mathrm{O}$. The final supernatants were filtered by $0,8-\mathrm{mm}$ pore-sized syringe filters, and the aliquots 
were stored at -20 ${ }^{\circ} \mathrm{C}$ until analysed. MMP-9 was measured by the quantitative enzyme linked immunosorbent assay described above.

\subsubsection{Measurement of faecal calprotectin}

In the second part of this study, faecal calprotectin was measured in a subset of UC patients ( $n=24$, male/ female: 10/14, mean age: 45,7 years). For calprotectin measurements, faecal specimens were thawed at $48{ }^{\circ} \mathrm{C}$ and calprotectin level was determined by a quantitative lateral flow assay (Quantum Blue, Bühlmann Laboratories, Schönenbuch, Switzerland) according to the manufacturer's instructions.

\subsubsection{Statistical analysis in the human studies}

The results are presented as mean \pm standard deviation. Due to the non-normal distribution of the data, a Kruskal-Wallis test was used to compare faecal MMP-9 levels of the three groups (control, IBS-D, and UC) four different groups of UC related to disease activity, and control, hyperplastic polyp, adenoma and CRC. Correlation between MMP-9 levels and disease activity scores or blood test results were determined by Spearman correlation. Correlation between MMP-9 and calprotectin were also tested by Spearman correlation. The cut-off levels, specificity, and sensitivity were calculated using receiver operating characteristic (ROC) analysis in both data groups to determine the predictive power of the MMP-9 level. In UC patients, active disease has been determined as total Mayo score $>3$ and Mayo endoscopic sub-score $>0$. The cut-off levels were calculated in case the area under the ROC curve (AUC) value was above 0,9 in UC study and 40,8 in the cancer study. The cut-off level was determined by the maximum value of Youden's index (sensitivity+ specificity-1). MMP-9 levels of high- and low-risk adenoma subgroups were analysed by the Mann-Whitney test. The different subgroups according to tumour localisation or stage were compared with the Kruskal-Wallis test. Differences were considered statistically significant 
when $\mathrm{P}<0.05$. Statistical analyses were performed by the $\mathrm{R}$ (version 3.1.2) statistical software (https://www.r-project.org/).

\section{RESULTS}

\subsection{Animal studies}

\subsubsection{In vivo and in vitro permeability measurements}

To define the impact of modestly-increased intestinal permeability, we studied transgenic mice with intestinal epithelial-specific constitutively-active myosin light chain kinase expression. This MLCK-dependent tight junction regulation increased intestinal permeability (Figure $1 \mathrm{~A}$ and $\mathrm{B}$ ). (In vitro permeability measurement: Mean \pm SD: WT jejunum: 0,8165 $\pm 0,4476 \mathrm{nmol} / \mathrm{h} / \mathrm{cm} 2 ; C A M L C K T g$ jejunum: 1,528 $\pm 0,5516 \mathrm{nmol} / \mathrm{h} / \mathrm{cm} 2$; $\mathrm{p}=0,0411$, In vivo measurement: Mean \pm SD: WT:11,83 $\pm 4,343, C A M L C K T g: 15,59 \pm 5,622$, $\mathrm{p}=0,0245)$.

\subsubsection{Body weight gain and intestinal transit}

Postnatal growth (Figure $1 \mathrm{C}$ ), reproduction, intestinal transit (Figure $1 \mathrm{D}$ ) are unaffected in CAMLCK transgenic (CAMLCKTg) mice. (Transit results, geometric centers Mean \pm SD: WT: 8,248 $\pm 0,6189 \mu \mathrm{g} / \mathrm{ml}$, CAMLCKTg: 8,595 $\pm 0,5105 \mu \mathrm{g} / \mathrm{ml}, \mathrm{p}=0,1988)$.

\subsubsection{Microbiota analysis}

We initially analysed the gut microbiome of 31 WT and CAMLCKTg pups born to 8 WT dams. The microbiomes segregated by pup genotype but not dam (Figure $1 \mathrm{E}$ ) and included increased Clostridium and decreased Bacteroidetes, Enterococcus spp, and 
Prevotella in CAMLCKTg mice (Figure $1 \mathrm{~F}$ ). Increased intestinal permeability can therefore cause dysbiosis-like microbiome shifts.

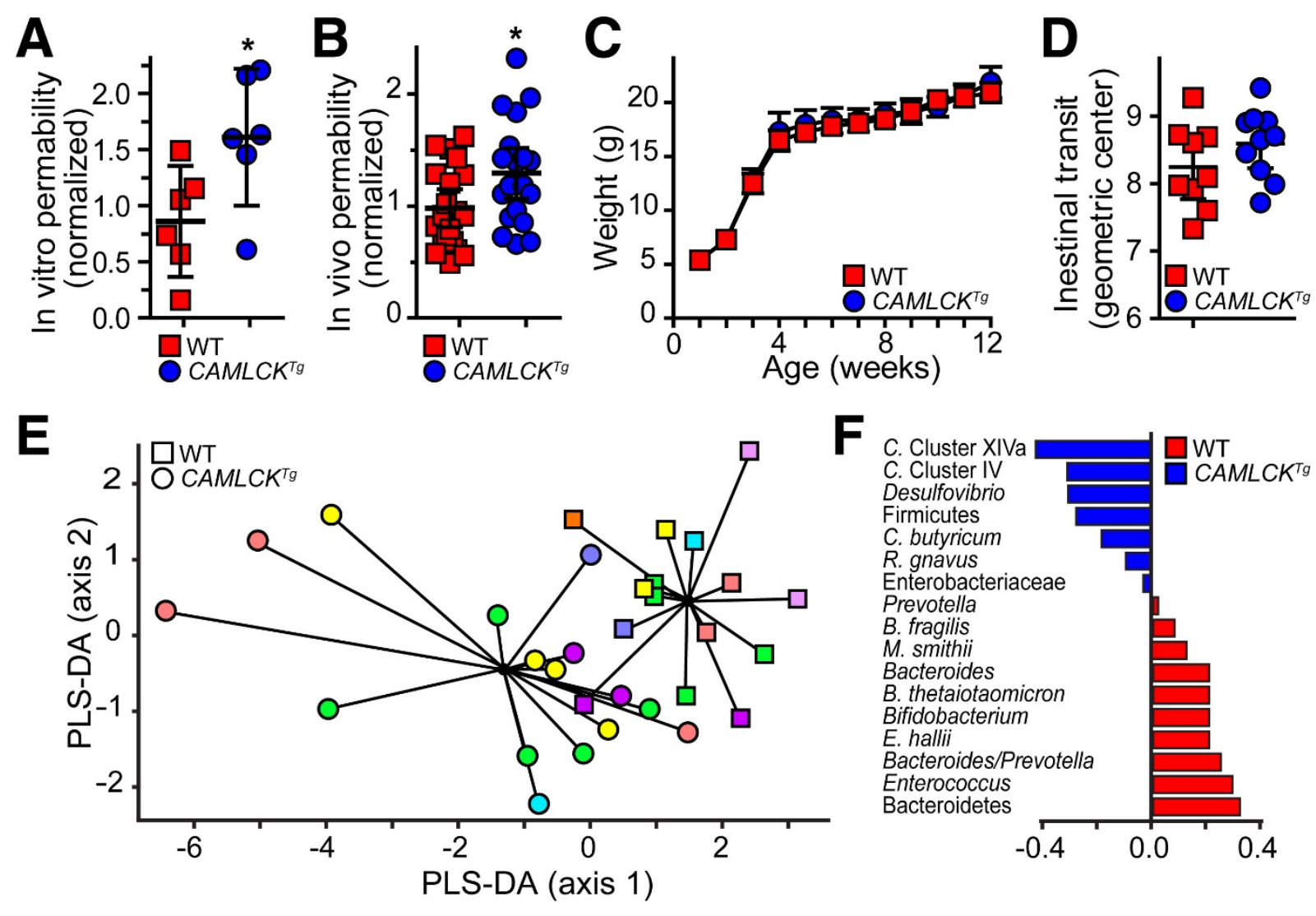

Figure 1: Trans-jejunal fluorescein flux was increased in CAMLCKTg (blue circles) relative to WT (red squares) littermates. mean $\pm \mathrm{SD}$; *, $p<0.05$, Mann-Whitney U test.

B. In vivo analysis using FITC-4kDa dextran demonstrated increased permeability of CAMLCKTg (blue circles, $\mathrm{n}=19$ ) relative to $\mathrm{WT}$ (red squares, $\mathrm{n}=20$ ) littermates. mean $\pm \mathrm{SD}$; *, $p<0.05$, t-test.

C. Weight gain was similar in WT (red squares, $\mathrm{n}=6$ ) and CAMLCKTg (blue circles, $\mathrm{n}=6$ ) littermates. mean $\pm \mathrm{SD}$.

D. Intestinal transit was similar in WT (red squares, $\mathrm{n}=10$ ) and CAMLCKTg (blue circles, $\mathrm{n}=9$ ) littermates. mean \pm SD.

E. Partial least squares discriminant analysis (PLS-DA) score plot based on the relative abundances of 18 microbial taxa in gut contents of CAMLCKTg (circles, $\mathrm{n}=16$ ) and WT (squares, $\mathrm{n}=15$ ) born to 8 different dams (each colour represents one dam).

F. Relative abundances of microbial communities in CAMLCKTg (blue) and WT (red) mice. Diagrams indicate regions analysed. 


\subsubsection{Open field test}

Both the percentage of distance travelled in the center and the fraction of time spent in the center of the open field were reduced in CAMLCKTg mice (Figure 2C); this did not reflect reduced locomotor activity, as total distance travelled in the entire area was similar in CAMLCKTg and WT mice (Figure 2C). (Time in center: (mean \pm SEM) WT: 10,18 \pm 1,219\% , CAMLCKTg: 5,785 \pm 1,312\%, $\mathrm{p}=0,0278$, distance in center: WT: 12,22 \pm 0,7487\%, CAMLCKTg: 7,860 \pm 0,7869\%, p=0,0011, total distance travelled: WT: 3602 $\pm 302,8 \mathrm{~m}$, CAMLCKTg: 4260 $\pm 419,8 \mathrm{~m}, \mathrm{p}=0,2157)$

\subsubsection{Colorectal distension}

CAMLCKTg mice displayed striking visceral analgesia to colorectal distension relative to WT littermates (Figure 2D). Sensitivity was restored by enzymatic MLCK inhibition, water avoidance stress, or naloxone-mediated opioid receptor antagonism (Figure 2D).

\subsubsection{C-Fos analysis}

C-Fos immunolabeling, an indicator of neuronal activity, was significantly greater in the paraventricular nucleus of the thalamus, the paraventricular nucleus of the hypothalamus, and the hippocampus, but not the medial prefrontal cortex, nucleus accumbens, or amygdala, of CAMLCKTg, relative to WT, mice (Figures. 3, 4). (Mean \pm SEM, paraventricular nucleus of the thalamus:WT: $102,4 \pm 27,49$ cells/mm², CAMLCKTg: $204,1 \pm 29,23$ cells $/ \mathrm{mm}^{2} \mathrm{p}=0,0297$, paraventricular nucleus of the hypothalamus: WT: $41,27 \pm 11,57$ cells $/ \mathrm{mm}^{2}$, CAMLCKTg: 114,2 $\pm 28,41$ cells $/ \mathrm{mm}^{2}, \mathrm{p}=0,0387$, hippocampus: WT: $24,50 \pm 2,397$ cells/mm², CAMLCKTg:

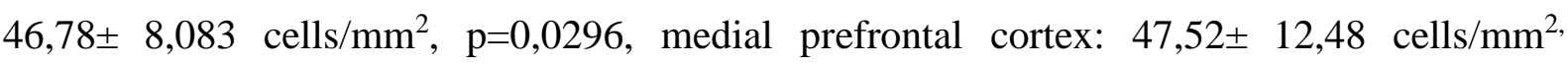
CAMLCKTg: 53,33 \pm 13,41 cells $/ \mathrm{mm}^{2}, \mathrm{p}=0,7577$, nucleus accumbens: WT: 55,36 $\pm 12,30$ 
cells/mm², CAMLCKTg: 70,82 \pm 12,93 cells $/ \mathrm{mm}^{2}, \mathrm{p}=0$ 0,4068, amygdala: WT: 65,98 \pm 24,16 cells/mm², CAMLCKTg: 91,98 \pm 23,87 cells $/ \mathrm{mm}^{2}, \mathrm{p}=0,4616$ )
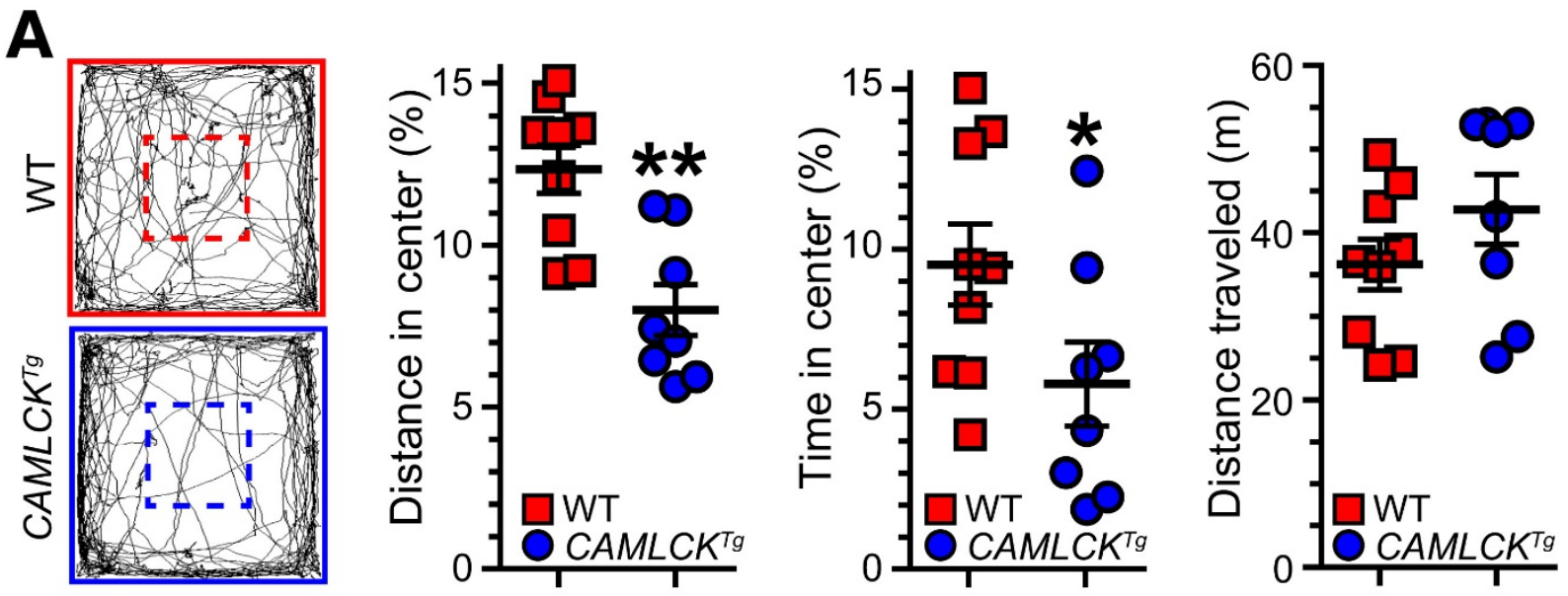

B

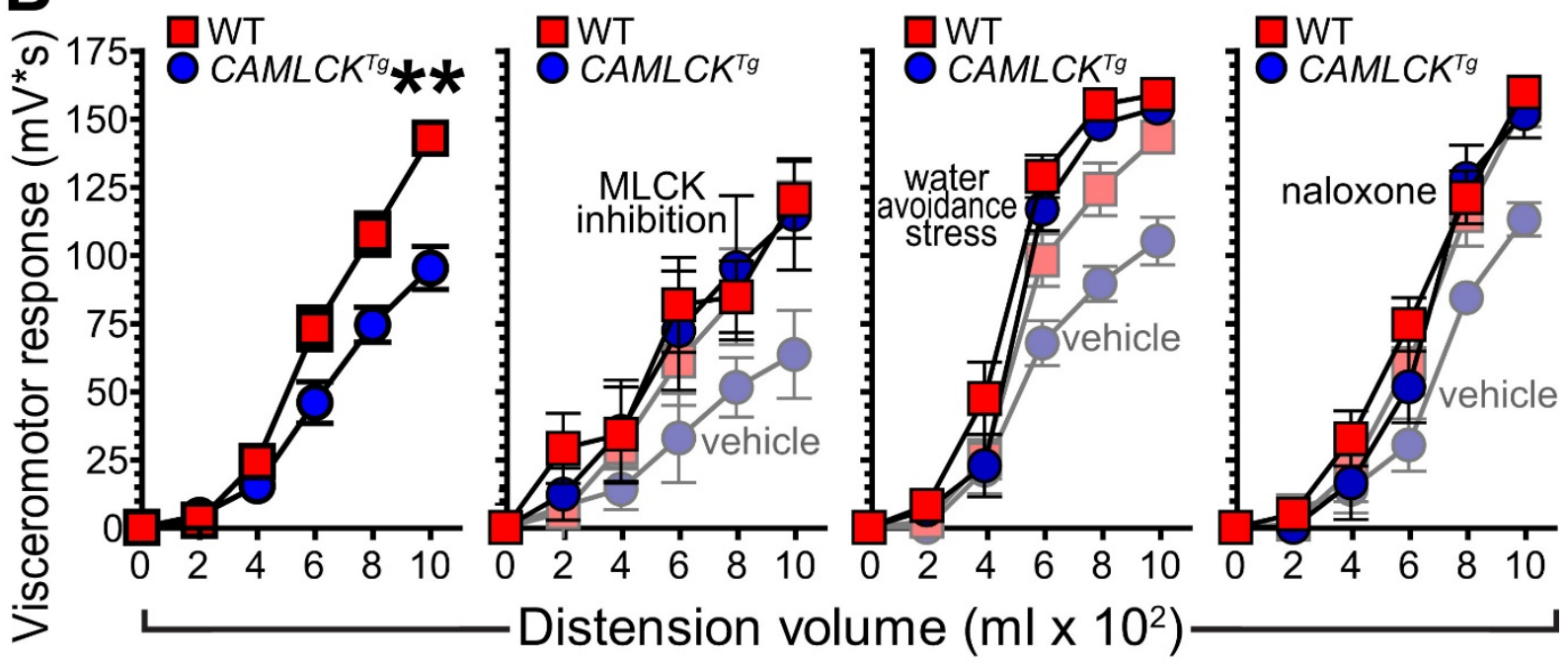

Figure 2: Increased intestinal permeability modifies behaviour and visceral sensitivity. A. Video tracking paths of representative WT and CAMLCKTg mice in the open field test. Percent distance travelled in the center (dashed lines), percent time in the center, and overall distance travelled in the entire field are shown. CAMLCKTg (blue circles, $\mathrm{n}=8$ ) and WT (red squares, $\mathrm{n}=9$ ) littermates were tested. mean $\pm \mathrm{SEM}$. *, $p<0.05$; **, $p<0.01$, Mann-Whitney U test. B. Stepwise colorectal distension-induced visceromotor responses in CAMLCKTg (blue circles, $n=7$ ) were reduced relative to WT (red squares, $n=7$ ) littermates. Genotype-specific differences were eliminated by MLCK inhibition, water avoidance stress, or naloxone treatment. $\mathrm{n}=5-9$ per condition; for each treatment (vehicle control CAMLCKTg and WT mice from the same experiment are shown with pale symbols in the last three graphs). mean \pm SEM; **, $p<0.01$, 2-way ANOVA. 


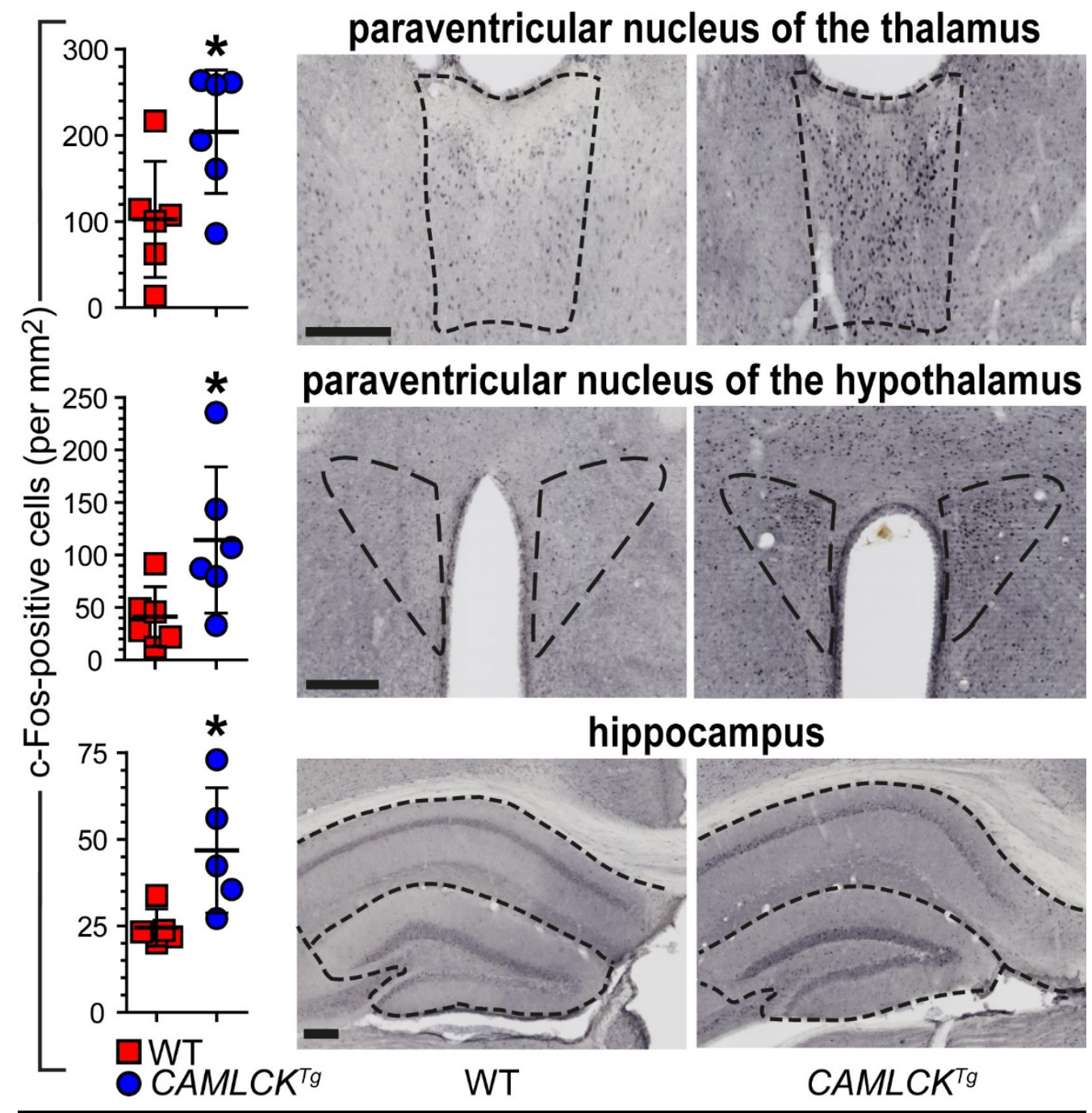

Figure 3: Increased intestinal permeability induces increased C-Fos immunolabelling in selected brain regions. CAMLCKTg (blue circles, $\mathrm{n}=5-6$ ) and WT (red squares, $\mathrm{n}=5-6$ ) littermates. Representative images of C-Fos immunolabeled brains from $C A M L C K T g$ and WT mice. Bars $=200 \mu \mathrm{m}$; mean $\pm \mathrm{SEM}$; * $p<0.05$, t-test. 


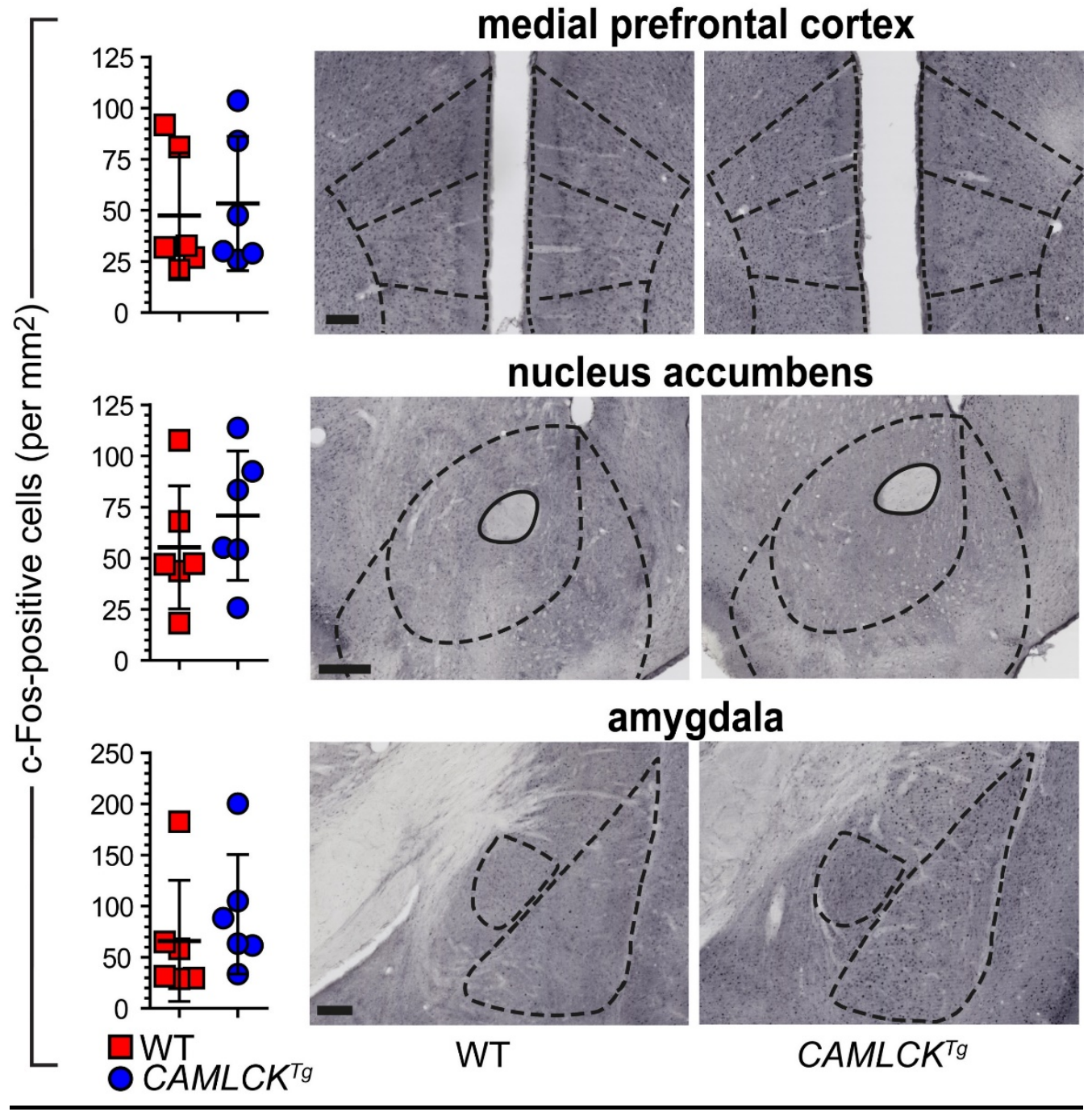

Figure 4: CAMLCKTg (blue circles, $n=5-6$ ) and WT (red squares, $n=5-6$ ) littermates. Representative images of C-Fos immunolabeled brains from CAMLCKTg and WT mice.

\subsection{Human studies}

\subsubsection{Validation assay of faecal MMP-9 measurement}

The concentrations measured in $\mathrm{ng} / \mathrm{mL}$ are shown in Figure 5. The figure clearly shows a linear concentration change corresponding to dilution. It indicates that (i) the faecal 
matrix itself has no influence on MMP-9 determination; and (ii) the kit from R\&D Systems is able to determine MMP-9 from human faecal extracts.

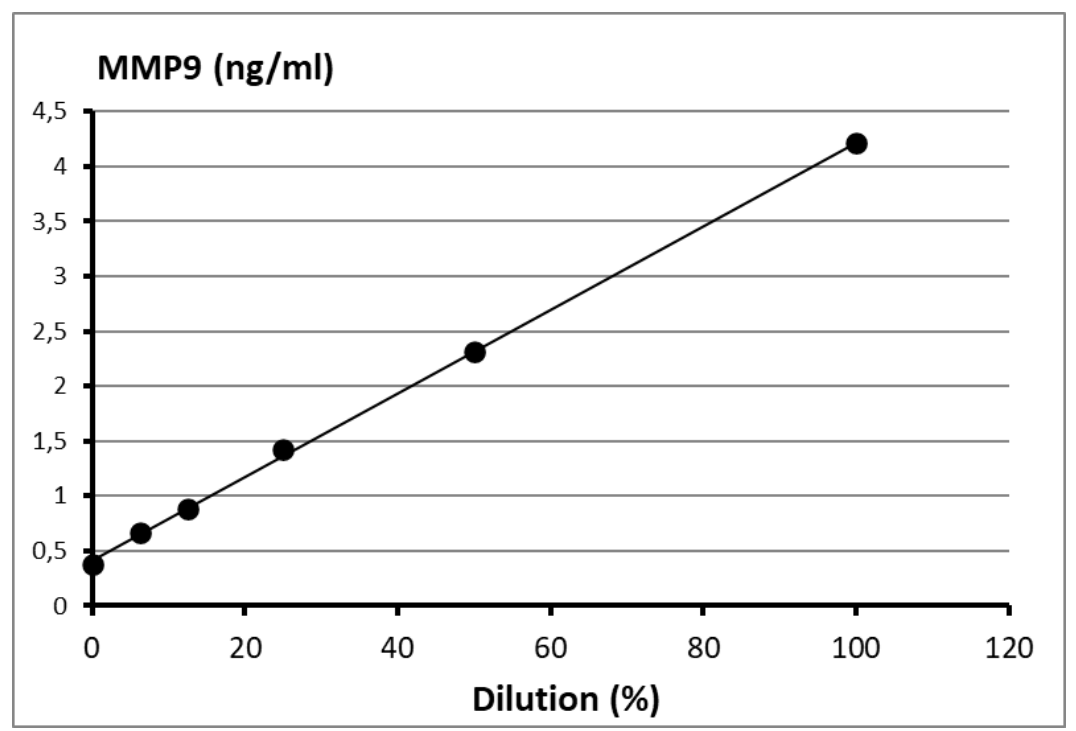

Figure 5: MMP-9 concentration levels at different dilutions in the validation assay.

\subsubsection{Faecal MMP-9 levels in control, IBS-D and UC patients' samples}

There were no significant differences between the three groups (control, IBS-D, and UC) regarding age $(\mathrm{P}=0,916)$. Faecal MMP-9 contents were significantly different between the three groups (Figure 6, $\mathrm{P}<0,001$ ). Indeed, specimens originating from healthy subjects had very low MMP-9 levels; nearly half of them were found to be below the detection limit of the assay (mean: 0,0208 $\pm 0,045 \mathrm{ng} / \mathrm{mL}$ ). Similarly, IBS-D patients had low faecal MMP-9 levels (IBS-D: 0,0017 $\pm 0,008 \mathrm{ng} / \mathrm{mL}$ ). However, in the UC group the average MMP-9 level was significantly higher (UC: 15,65 $\pm 20,14 \mathrm{ng} / \mathrm{mL}$ ). To distinguish UC patients from healthy controls or IBS-D patients, the cut-off level was determined at 0,245 ng/mL (AUC=0,939). By means of this cut-off, sensitivity was $85.1 \%$ and specificity 99,99\% (Figure 6) 


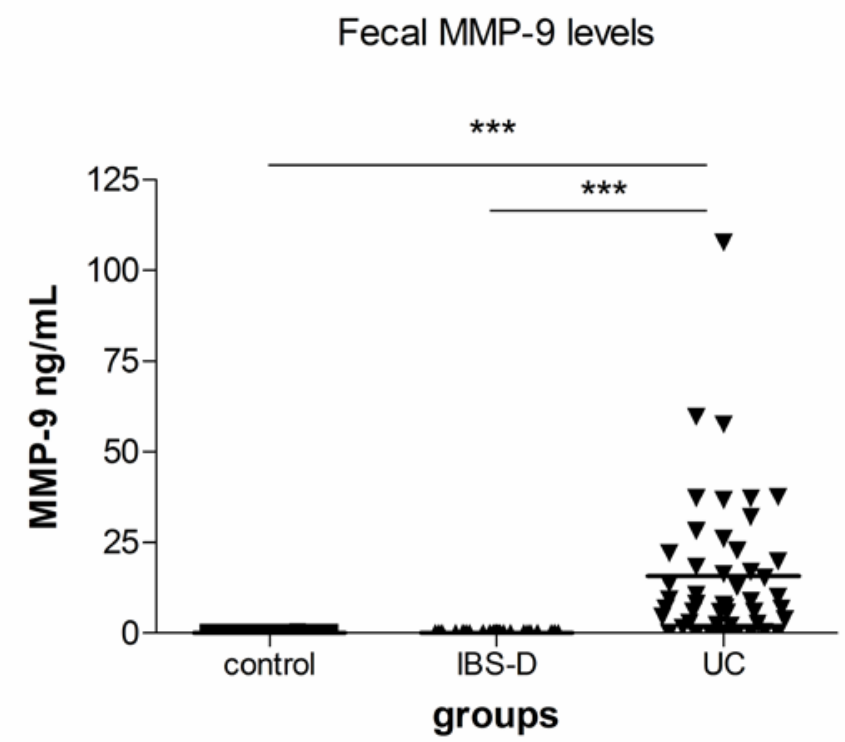

Figure 6: Faecal MMP-9 levels in the three different groups: control, IBS-D, UC. Faecal MMP-9 concentration is significantly elevated in UC patients compared to controls or IBS-D patients $\left({ }^{* * *} \mathrm{p}<0,001\right)$.

\subsubsection{Correlation between faecal MMP-9 levels with disease activity in UC}

MMP-9 activity significantly correlated with the overall Mayo score $(\mathrm{R}=0,616$; $\mathrm{p}<0,001$; AUC=0,905). Faecal MMP- 9 levels were significantly lower in case of inactive disease compared to moderately or highly active (Figure 7.). Further, within the components of the Mayo score a strong correlation was observed with the endoscopic evaluation of the colorectal mucosa $(\mathrm{R}=0,653 ; \mathrm{P}<0,001$, AUC=0,900). To separate active UC patients from those in remission, the cut-off level of MMP-9 was determined at $2.38 \mathrm{ng} / \mathrm{ml}$. This cut-off resulted in a sensitivity of $97.1 \%$ and a specificity of $83.3 \%$. Correlation of faecal MMP-9 with laboratory findings is illustrated in Table 1. 


\section{Fecal MMP-9 in UC patients}

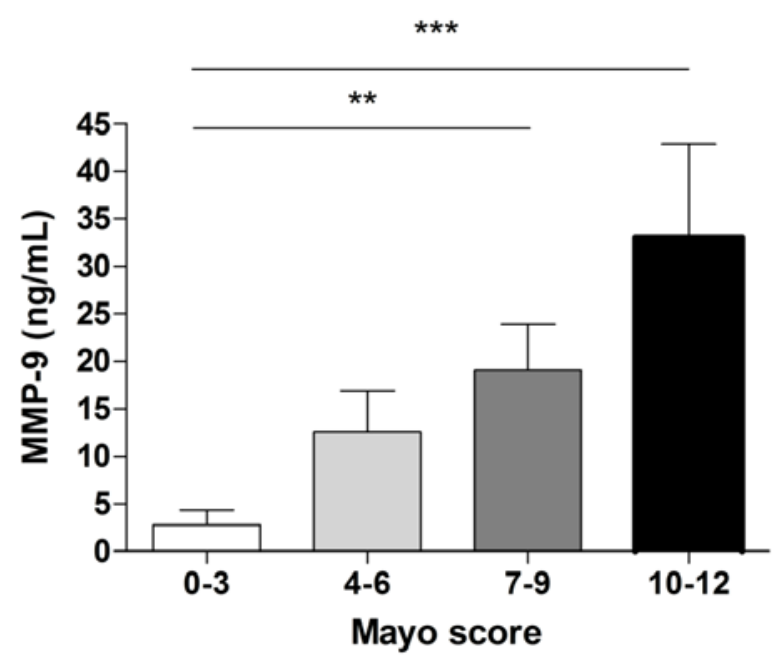

Figure 7: Faecal MMP-9 levels in UC patients depending on disease activity. Faecal MMP-9 concentration is significantly elevated in UC patents with moderate $\left({ }^{* *} \mathrm{p}<0,01\right)$ and severe $(* * * \mathrm{p}<0,001)$ activity compared to those in remission.

\begin{tabular}{|c|c|c|c|c|c|c|}
\hline $\begin{array}{l}\text { Blood } \\
\text { test } \\
\text { result }\end{array}$ & CRP & $\begin{array}{l}\text { Erythrocyte } \\
\text { sedimentation } \\
\text { rate }\end{array}$ & $\begin{array}{c}\text { Serum } \\
\text { ferrous } \\
\text { level }\end{array}$ & $\begin{array}{l}\text { White } \\
\text { blood cell } \\
\text { count }\end{array}$ & Haemoglobin & platelet \\
\hline MMP-9 & $\begin{array}{l}\mathrm{R}=0,456 \\
\mathrm{P}=0,002\end{array}$ & $\begin{array}{l}\mathrm{R}=0,228 \\
\mathrm{P}=0,137\end{array}$ & $\begin{array}{l}\mathrm{R}=0,454 \\
\mathrm{P}=0,003\end{array}$ & $\begin{array}{l}\mathrm{R}=0,404 \\
\mathrm{P}=0,005\end{array}$ & $\begin{array}{l}\mathrm{R}=0,151 \\
\mathrm{P}=0,317\end{array}$ & $\begin{array}{l}\mathrm{R}=0,296 \\
\mathrm{P}=0,046\end{array}$ \\
\hline
\end{tabular}

Table 1. Correlation of faecal MMP-9 levels with blood test results. Significance is accepted at $\mathrm{p}<0,05$.

\subsubsection{Correlation of faecal MMP-9 concentration with faecal calprotectin levels in UC}

In a subset of 24 UC patients, faecal calprotectin levels were also determined. Significant correlation was found between the faecal MMP-9 and calprotectin levels $(\mathrm{R}=0,495 ; \mathrm{P}=0,014)$. In this subset of patients, the correlation of endoscopic and overall Mayo scores was calculated with both MMP-9 and calprotectin. In this smaller cohort of patients, faecal MMP-9 levels showed a slightly stronger correlation with the endoscopic component of 
the Mayo score than calprotectin $(\mathrm{R}=0,587 ; \mathrm{P}=0,003$, $\mathrm{AUC}=0,895$ for calprotectin and $\mathrm{R}=0,653 ; \mathrm{P}=0,002$, AUC=0,939 for MMP-9), and a higher correlation with the overall Mayo score than calprotectin $(\mathrm{R}=0,587 ; \mathrm{P}=0,016$, $\mathrm{AUC}=0,895$ for calprotectin and $\mathrm{R}=0,718$; $\mathrm{P}=0,002$, AUC $=0,905$ for MMP-9).

\subsubsection{Faecal MMP-9 levels in groups of non-adenoma/adenoma/CRC patients}

Seventeen patients (male/female: 8/9; mean age: 59,7 years) were found negative on total colonoscopy and 17 patients had no other finding from non-inflamed diverticulosis (male/female: 3/14; mean age: 71,8 years). There was no significant difference between these two groups in terms of faecal MMP-9 levels $(\mathrm{P}=0,49)$, therefore these 34 patients were used as polyp-free/non-malignant controls (mean age: 65,7 years). Out of the other patients, 15 had hyperplastic polyps (male/female: 9/6; mean age: 62,3 years), 32 had adenomas (male/female: 21/11; mean age: 67,0) and 28 had CRC (all adenocarcinomas; male/female: 17/11; mean age: 67,2 years) according to the endoscopic and histological examinations. There were no statistically significant differences between the four groups (controls, hyperplastic polyps, adenomas, CRC) regarding the age. Faecal MMP-9 levels were significantly different between the four groups (Figure 8, $\mathrm{P}<0,0001$ ). Faecal samples from control patients had very low MMP-9 levels (mean: 0,118 $\pm 0,057 \mathrm{ng} / \mathrm{mL}$ ). Patients with hyperplastic polyps also presented with low faecal MMP-9 levels (mean: 0,112 $\pm 0,073 \mathrm{ng} / \mathrm{mL}$ ). Adenoma patients had slightly, but not significantly elevated faecal MMP-9 levels (mean: 0,656 $\pm 0,396 \mathrm{ng} / \mathrm{mL}$ ). Nevertheless, patients with CRC had a significantly increased mean of faecal MMP-9 levels (mean: 7,028 $\pm 3,722 \mathrm{ng} / \mathrm{mL}$ ). To distinguish CRC patients from controls, the cut-off level was determined at 0,23 ng/mL (AUC=0,913; 95\% CI: 0,833-0,994; $\mathrm{P}<0,001$ ). This cut-off level was associated with a sensitivity of $89,3 \%$ and a specificity of $91,2 \%$ (Figure 9). Patients with hyperplastic polyps (AUC=0,526; 95\% CI: 0,340-0,711; $\mathrm{P}=0,779$ ) were not distinguishable from control individuals by ROC analysis. Among adenomas, 22 out of 32 were at increased risk of malignancy, with a mean faecal MMP-9 level significantly elevated compared with low-risk adenomas (mean of high-risk adenomas: 0,936 $\pm 0,570$ vs mean of low-risk adenomas: 0,040 $\pm 0,017$; $\mathrm{P}<0,05$; Figure 10). Low-risk adenomas could not be distinguished 
from controls by faecal MMP-9 levels (AUC=0,456; 95\% CI: 0,236-0,676; $\mathrm{P}=0,706$ ) When ROC analysis was performed with an aim to select high-risk adenomas and CRC from controls, the cut-off level was determined at $0.085 \mathrm{ng} / \mathrm{ml}$. In this case, sensitivity was $76 \%$ and specificity was 85,3\% (AUC=0,806; 95\% CI: 0,710-0,902; $\mathrm{P}<0,001)$. With this lower cut-off level, 59\% of high-risk adenomas were identified. By ROC analysis of high-risk adenomas vs controls, the cut-off level would be similarly $0.085 \mathrm{ng} / \mathrm{ml}^{-1}$ (AUC=0,670; 95\% CI: 0,507-0.833; $\mathrm{P}=0,033)$.

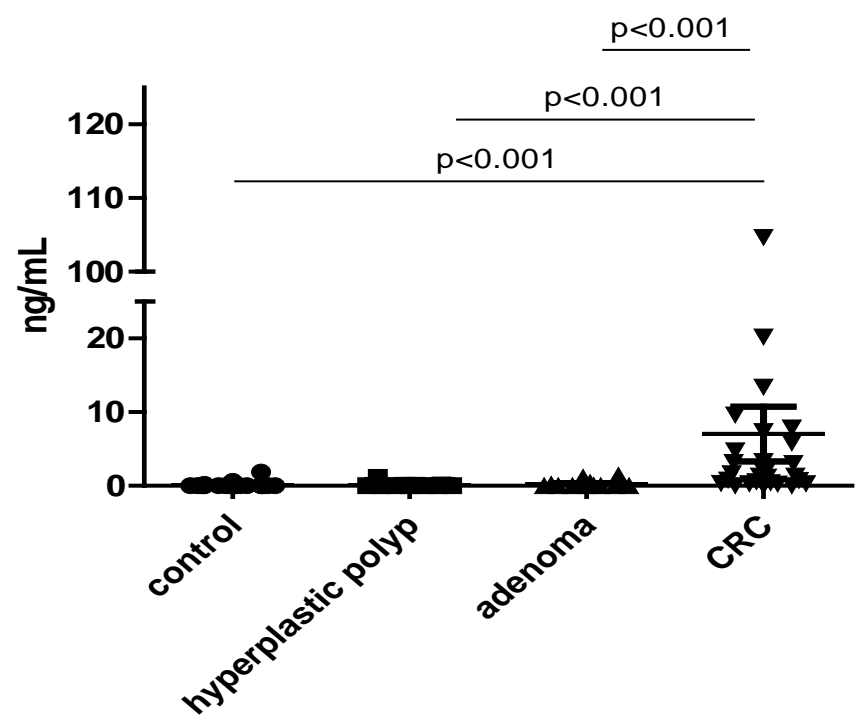

Figure 8: Faecal MMP-9 levels of the different groups (based on the colonoscopy and histology). Abbreviation: $\mathrm{CRC}=$ colorectal carcinoma 


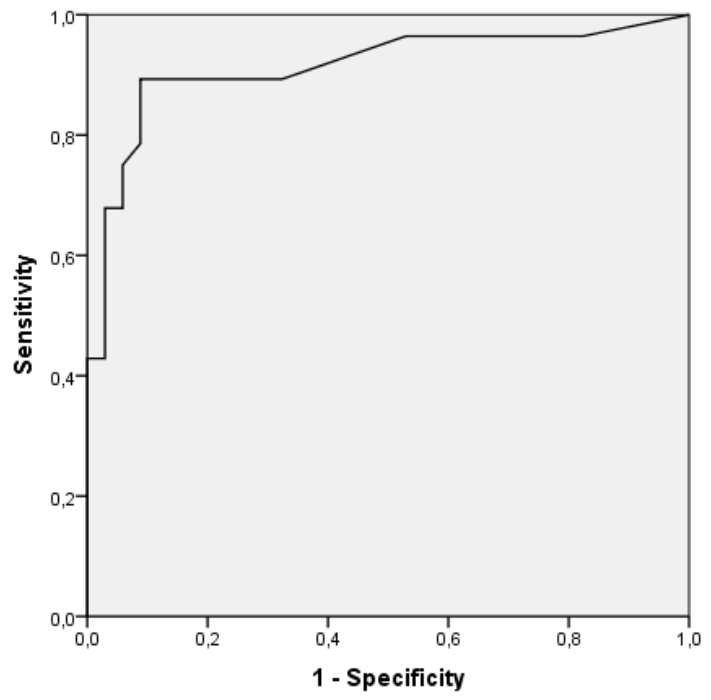

Figure 9: ROC (receiver operating characteristic) curve of CRC vs. controls

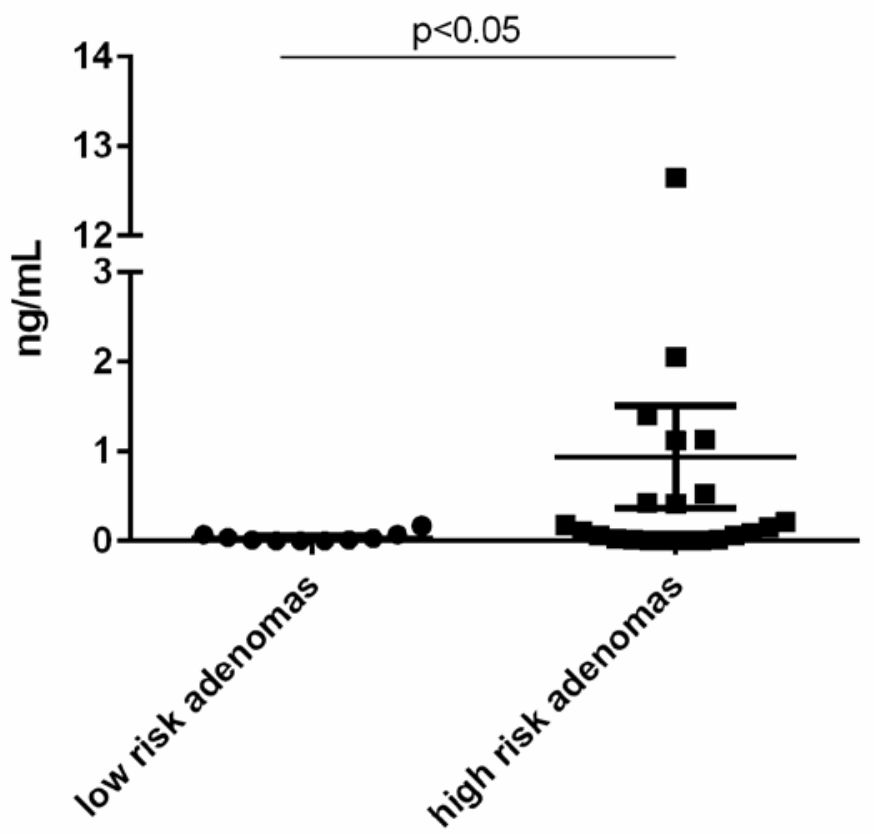

Figure 10. Faecal MMP-9 levels in low-risk and high-risk adenomas. 


\subsubsection{Faecal MMP-9 levels according to CRC localisation and stage}

The number of patients regarding the site of the CRC was as follows: rectum: 14, sigmoid colon: 6, lineal flexure: 1 , transverse colon: 2 , hepatic flexure: 2 , ascending colon: 1 , and caecum: 2 patients. If faecal MMP-9 levels are analysed according to the site of the CRC, no significant difference was observed $(\mathrm{p}=0,146)$, although a trend was seen towards higher levels in case of a rectal tumour (mean: 12,33 $\pm 7,274 \mathrm{ng} / \mathrm{ml}$ ) compared with left-sided colon (lineal flexure-sigmoid colon; mean: $1.619 \pm 0.978 \mathrm{ng} / \mathrm{ml}$ ) and transverse-right-sided colon (from caecum to transverse colon; mean: 1.830 $\pm 0.656 \mathrm{ng} / \mathrm{mL}$; Figure 11A). According to Dukes' classification, no significant difference could be seen between the groups (Figure 11B; $\mathrm{p}=0,236)$. 


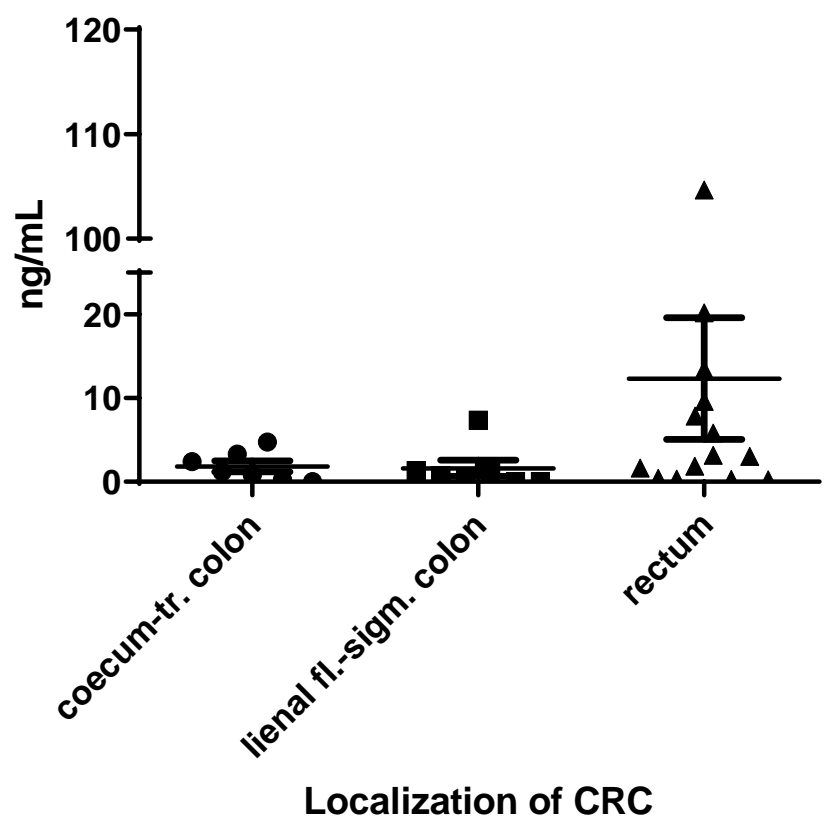

A

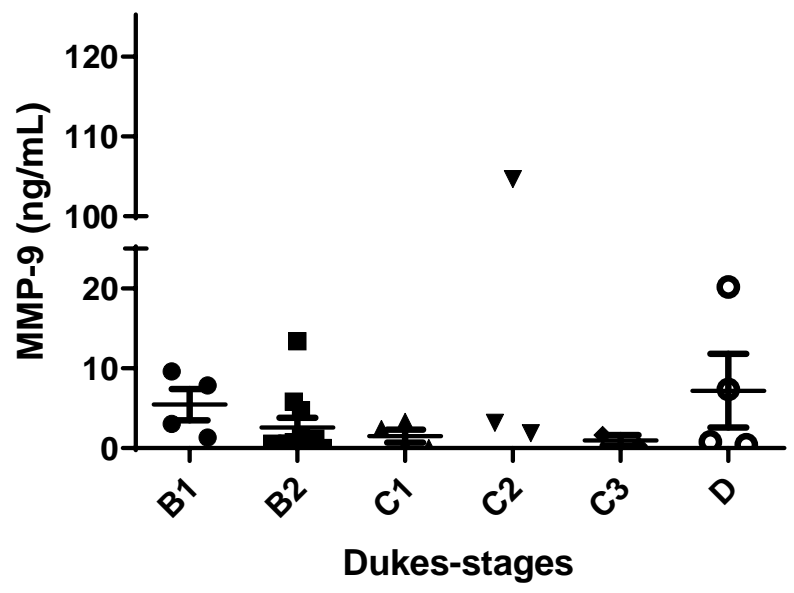

B

Figure 11: Faecal MMP-9 levels in CRC patients. Values according to (A) localisation and (B) Dukes' stages of CRC. 


\section{DISCUSSION}

\subsection{Animal studies}

Our results showed that mice exhibiting a permanent and discrete elevation of intestinal permeability are characterized by modifications of colonic microbiota composition. Moreover, CAMLCKTG animals exhibit a naloxone-sensitive visceral hyposensitivity to colorectal distension, associated with an anxiety-prone phenotype.

In the present work we have used a transgenic mouse that expresses a constitutively active myosin light chain kinase. MLCK is expressed throughout the gut epithelium and controls the cytoskeleton contraction through MLC phosphorylation upon TNF stimulation [47]. CAMLCKTg animals exhibit discrete increased permeability, without alterations of tight junction organization, associated with low-grade mucosal inflammation evoked by increased expression of TNF $\alpha$, IFN $\gamma$, IL-10 and TGF $\beta$ [15]. However, transgenic mice grew normally and did not present any modification of the gastrointestinal transit. We have confirmed that CAMLCKTg mice display an increased permeability in vivo, which seems limited to the small intestine as shown by Ussing chambers experiments. This observation is consistent with the higher expression of the transgene denoted in the jejunum compared with the colon [15].

Microbiota analysis has been performed in colonic samples from WT and CAMLCKTg animals using Gut low density Array (GuLDA) developed by Bergström [44], allowing the determination of abundancy of targeted species using 16S rRNA. It is important to note that we performed this analysis on littermates, i.e. on animals from the same dam and bred together until weaning. We have observed an increased abundancy of Firmicutes, especially Clostridium clusters XIVa and IV, which encompass bacterial species able to adhere and degrade intestinal mucus [48]. Among them, Ruminococcus gnavus has been shown to degrade mucins through a specific bacterial adhesion molecule that also present a sialydase activity [49]. In addition, higher relative levels of Desulfovibrio spp, a strain known to bind mucins, especially in a context of inflammation [50], have also been denoted in transgenic animals. Of interest, Akkermansia muciniphila was not detected in both genotypes, while we have found these bacteria in older mice (data not shown). On the other hand, transgenic mice 
microbiota is characterized by a lower abundancy of Bacteroidetes. This leads to an increase in the Firmicutes/Bacteroides ratio, which has also been observed in IBS patients [51], despite the clinical relevance of this parameter remains a matter of debate.

Based on our observations CAMLCKTg, animals display a phenotype close to what could be observed in IBS patients [52], in terms of epithelial barrier dysfunction, mucosal immune alterations and dysbiosis. The diagnosis of IBS relies on a symptom-based approach that identifies, according to Rome IV criteria, four subtypes: constipation, diarrhoea, mixed symptoms, and unsubtyped [1]. Altered intestinal barrier function is frequently observed whatever the IBS subgroup considered. Of interest, increased permeability has been characterized in biopsies harvested from patients suffering from IBS [53].Luminal contents of these patients are also likely involved in triggering epithelial barrier impairment since faecal supernatants promote increased permeability associated with occludin disruption [54]. In addition, alterations of the barrier function do not appear to be limited to the colon, but extend to the small bowel [55], suggesting the commitment of the whole gut in this phenomenon. Increased intestinal permeability has been suspected to be a prerequisite for the enhanced visceral pain experienced by patients. In humans, the severity of IBS symptoms in IBS-D patients is positively correlated to intestinal permeability measured by the lactulose/mannitol ratio [9]. In rats submitted to partial restraint stress, a valuable model to induce visceral hypersensitivity to colorectal distension associated with impaired intestinal barrier function, normalization of increased permeability by probiotics [56] or an MLCK inhibitor [7] resulted in visceral normosensitivity. Therefore, we hypothesized that CAMLCKTg mice, showing increased permeability, would also present visceral hypersensitivity to colorectal distension. Unexpectedly, we observed a marked, naloxone-sensitive hyposensitivity to colorectal distension for moderate to high volumes of distension applied. Visceral sensitivity can be negatively or positively modulated by descending pathways. Indeed, Wilder-Smith et al. have shown an abnormal activation of endogenous pain inhibitory pathways in IBS patients [57]. In rats, Larauche et al. have established that animals acutely exposed to water avoidance stress present analgesia to colorectal distension in females but not in males [58]. This analgesia was sensitive to naloxone. Conversely, one day after stress, female rats exhibited a visceral hyperalgesia. The authors hypothesize that the initial hypoalgesia could be linked with the engagement of antinociceptive pathways in response to anxiety of fearfulness associated with 
stress exposure. Therefore, we determined the levels of anxiety of CAMLCKTg and WT animals, and we observed that transgenic animals present an anxiety-prone phenotype evidenced by the open field. This suggests that the increased level of anxiety of transgenic animals compared with their WT littermates may determine a lower sensitivity to colorectal distension. Our works on neuronal activity in non-stressed animals show, that the permeability elevation due to the transgene induced a cerebral activity pattern, with altered activation in regions of the brain usually associated with visceral sensation and the affective response to pain. Literature data show, paraventricular thalamus as responsible for wakefulness [59], and in a latest post-traumatic stress response study similar regions alteration was observed (nucleus paraventricularis of the thalamus, medial prefrontal cortex, hypocampus) [60]. In CAMLCKTg transgenic mice, the modification of the intestinal permeability provoked an anxious behaviour through the central nervous system.

\subsection{Human studies}

To the best of our knowledge our study is the first to report elevated faecal MMP-9 levels in patients with active UC compared to functional (IBS-D) patients or controls. In UC patients faecal MMP-9 levels are excellent predictors of disease activity and show a significant correlation with the clinical and endoscopic scores. Faecal MMP-9 levels correlate significantly with faecal calprotectin, a well-known marker of disease activity in UC. MMPs are a family of Zn2+-containing endopeptidases capable of degradation of the extracellular matrix. They are secreted by various cell types, such as fibroblasts, mesenchymal cells, tumour cells, and several inflammatory cells like monocytes, lymphocytes, and neutrophils. MMP-9 is a gelatinase that represents $\approx 30 \%$ of protein content of neutrophil granules [61]. In a murine experimental model, it has been shown that epithelial cells and granulocytes are the main sources of MMP-9 in colitic tissue, and exogenous MMP-9 impairs epithelial wound healing and cell attachment in vitro [62]. Urinal MMP-2 and MMP-9 levels have been suggested to discriminate children with IBD from controls; nevertheless, urine samples were found positive for MMP-2 in 25\%, for MMP-9 in 20\% of control individuals, leading to false positivity in the diagnosis [34]. Further, as MMPs play important roles in cancer development 
and metastasis formation[63], urinary MMPs were found elevated in various cancer types including prostate, bladder, breast, brain, and hematologic malignancies [64-67]. These findings point out that urinary MMPs are not specific for IBD. We hypothesize that faecal MMP level may be more characteristic of gastrointestinal pathologies than of other organspecific or systemic disorders, although its specificity to UC compared to other colon diseases needs to be verified. In colonic biopsies, MMP-9 levels were significantly increased in UC patients, and less significantly increased in CD patients compared to controls [33]. Based on colonic biopsies, a recent study demonstrated that in contrast to UC, MMP-9 is not involved in the inflammatory process of lymphocytic colitis and collagenous colitis [68]. By means of a cut-off level of faecal MMP-9 at 0,245 ng/mL, none of our controls and IBS-D patients tested positive. Nevertheless, faecal MMP-9 negativity does not rule out IBD, as seven UC patients, all in remission, were also below the cut-off level. It is important to note that none of them had colonic ulcerations on endoscopy (Mayo endoscopic subscore $\leq 1$ ). Therefore, our data suggest that low faecal MMP-9 level is a negative predictor of mucosal lesions. Faecal MMP-9 positivity suggests the need of further evaluation of inflammatory diseases or other gastrointestinal pathologies. Interestingly, in an earlier study on CD patients, MMP-9 plasma levels were found elevated compared to controls, but no correlation could be observed with the Crohn's Disease Activity Index (CDAI) [69]. Similarly, it has been demonstrated that other disease activity markers, such as calprotectin [70], lactoferrin, or CRP, and even endoscopic appearance correlate poorly with CDAI [71]. In our study, however, faecal MMP9 concentration showed a significant correlation with the clinical and endoscopic scores in UC patients. Additionally, faecal MMP-9 level significantly correlated with CRP, a laboratory value referring to inflammation, widely used as a disease activity marker in the blood. In the last part of our study we compared faecal MMP-9 to an established faecal marker in UC, calprotectin, and a significant correlation was found between them. In some centres, faecal calprotectin is already used as a routine test to discriminate IBS from IBD[72]. These data suggest that faecal MMP-9 can be a novel marker to help in the differential diagnosis of patients with diarrhoea and abdominal pain. Further, it could be a new complementary noninvasive method to assess mucosal inflammation and lesions in UC patients.

To the best of our knowledge, we report for the first time that MMP-9 levels are elevated in faecal specimens from CRC patients compared with patients without polypoid 
lesions. Furthermore, faecal MMP-9 can be used to distinguish CRC patients from patients with no polypoid lesions, with a high sensitivity and specificity. Additionally, with a lower cut-off level, faecal MMP-9 is able to identify nearly $60 \%$ of patients with a high-risk adenoma. MMP-9 is a gelatinase capable of the degradation of the extracellular matrix, which is believed to have a role in the progression and metastasis formation of many tumours, including colon cancer [43]. Apart from colon cancer cells, tumour-infiltrating neutrophils are also an important source of MMP-9, as a major angiogenesis-inducing factor [73, 74]. Serum MMP-9 was suggested as an early non-invasive biomarker for breast cancer [75] and a prognostic marker for lung cancer [76]. Urinary MMPs were found elevated not only in bladder, renal and prostate cancers but also in anatomically distant tumours, including breast, brain, and haematological malignancies [64-67]. Recently, significant efforts have been made to explore the potential role of MMP-9 as a biomarker in CRC. In the serum, it has been suggested as a biomarker of CRC in symptomatic patients [77], and its levels correlated with the Dukes' stage [78]. Furthermore, it has been recently shown that serum levels of neutrophil gelatinase-associated lipocalin in complex with MMP-9 are elevated in CRC patients, but it was found unsuitable as a diagnostic marker, as its discriminative power was very poor [79]. We have found the discriminative power of faecal MMP-9 levels in CRC patients sufficient, which can be explained by the fact that faecal MMP-9 could be more informative for gastrointestinal malignancies than urine or serum. Our method does not require a preceding diet, and one faecal sample gave a sufficient sensitivity for discrimination. Its analysis is automated, and the cut of concentration is adjustable. In terms of these characteristics, faecal MMP-9 measurement, similarly to FIT, is more convenient for clinical use than gFOBT. Furthermore, faecal MMP-9 measurement provides a higher sensitivity and specificity for CRC than gFOBT and many types of FIT. In the past decade, numerous faecal molecular markers have been described, which are based on the detection of genetic mutations and epigenetic alterations, linked to the carcinogenetic process [80]. Genetic markers are claimed to be more sensitive than FOBTs [80]. However, one disadvantage of these markers is that they are not present in every colorectal carcinoma. To overcome this problem, genetic markers are often combined in a panel, but this may lead to a decrease in specificity [80]. Furthermore, an important limitation of these methods is their high costs. Current evidence is not sufficient to accept the routine use of faecal genetic markers, and the European Guidelines for quality assurance have stated that they are currently unsuitable for CRC screening [40]. 
Similarly to molecular tests, our method also has the advantage over FOBTs in that it detects a marker originating directly from cancer, which, contrary to bleeding, is supposed to be permanent. However, the costs of faecal MMP-9 measurement are far beyond those of molecular tests, as they are $<10$ USD per sample, which is comparable to the costs of FOBT. This method is markedly cheaper than faecal DNA tests, which cost around 350 USD per sample [81]. The process of transformation from normal epithelium to cancer takes 10-15 years in most cases, leaving a possibility to remove precancerous lesions before they turn to definitive cancer [38]. Therefore, a reliable marker detecting high-risk adenomas is of high importance. The sensitivity of FIT for advanced adenomas is only around 20-33\% [82, 83]. DNA tests are characterised by a much higher sensitivity, for example, K-ras mutations are detected in the faeces of around 50\% of individuals with adenomas $>1 \mathrm{~cm}$ [84], p53 mutations in up to $64 \%$ of severely dysplastic polyps [85] and 4-26\% of adenomas [84]. Methods based on the detection of epigenetic changes appear to have a wide range of sensitivity, for example, the methylation of SFRP2 can be detected in the faeces of 52,4\% of individuals with adenomas [86], while the methylation of methylguanine DNA methyltransferase can be found in $36 \%$ of the faeces of adenoma patients but that of the human mut I homolog-1 in only $11 \%$ [87]. Faecal tumour M2 pyruvate kinase, a promising marker in CRC screening, showed a sensitivity of 22\% for advanced adenomas [88]. Serum MMP-9 levels were elevated not only in cancer but also in colorectal adenoma patients compared with healthy subjects [78]. Similarly, MMP-9 expression was found increased not only in tissue samples from CRC but also in those from highly dysplastic adenomatous polyps compared with hyperplastic polyps or adenomas with low dysplasia [89]. Furthermore, MMP-9 protein expression has been elevated in adenomas with high grade dysplasia compared with other adenomas or normal colonic tissue [90]. Our results have shown that faecal MMP-9 levels from high-risk adenoma patients are significantly elevated compared with patients with low-risk adenomas. However, with a lower cut-off level, our marker identified 59\% of high-risk adenoma patients as positive. This result is definitely higher than the usual sensitivity of FOBTs and comparable to that of molecular tests but without the high costs of the latter. We did not find any significant difference between the faecal MMP-9 levels of the groups with different tumour location. Nevertheless, a tendency could be seen towards the highest faecal MMP-9 levels in rectal adenocarcinoma patients. This is probably not related to the nature of rectal tumours but could be explained with a less time for degradation of the marker in the intestinal lumen, 
similarly to that seen by FIT [82]. Our study has some limitations. As our department is a specific colorectal centre, our patients were a selected population not representing the general population. Patients were on a low-fibre diet at the time of faecal sample collection, as a preparation for the upcoming colonoscopy. Data on the time of the day at faecal sample production was not collected in our study. Although the percentage of cancer and adenoma found on colonoscopy was relatively high, the number of patients in the different subgroups (i.e., tumour localisation, Dukes' stage) may be insufficient for the detection of minor differences. Moreover, MMP-9 is also elevated in gut inflammation, which may limit its use in case of, for example, IBD. We hypothesise that, in a more general population, elevated faecal MMP-9 levels in some cases could be related to inflammation or other cancers in the gastrointestinal tract. However, even in this case, the method would be a good predictor of the presence of a non-functional gastrointestinal disorder, based on our previous results showing that faecal MMP-9 measurement can well discriminate IBS-D patients and healthy subjects from UC patients. A further limitation is that, owing to the different cut off levels calculated on the same sample groups, the performance of the method may be overestimated. A further study with a larger and more general patient population could help us to clarify whether diet, time of the day at sampling, tumours of the upper GI tract and so on can affect faecal MMP-9 levels and could also serve to verify the performance of our method. A comparison with FIT on the same samples could be useful. Moreover, a larger study on a general cohort could also provide more information if this method is valuable for CRC screening. Nonetheless, our study was suitable to identify a new marker, faecal MMP-9, capable of discrimination of CRC from polyp-free controls. Faecal MMP-9 is characterised by many advantages of FIT and also of the molecular markers, without high costs. Further studies on a larger and more general population are needed to confirm whether this promising method is suitable for CRC screening as a complementary assay. 


\section{NEW RESULTS ESTABLISHED IN THE THESIS}

\subsection{Animal studies}

1. CAMLCKTg results discrete permeability elevation in the small intestine in mice. This modification does not change the body weight and intestinal transit, but causes dysbiosis-like microbiota composition, increased Clostridium and decreased Bacteroidetes, Enterococcus spp, and Prevotella compared to WT littermates.

2. Visceral sensitivity responses in CAMLCKTg mice are reduced relative to WT littermates. Genotype-specific differences are eliminated by MLCK inhibition, water avoidance stress, or naloxone treatment.

3. CAMLCKTg mice show behaviour modifications compared to WT littermates, intestinal permeability elevation causes anxiety-like phenotype.

4. Neuronal activity is significantly greater in the paraventricular nucleus of the thalamus, the paraventricular nucleus of the hypothalamus, and the hippocampus, but not the medial prefrontal cortex, nucleus accumbens, or amygdala in CAMLCKTg, relative to WT mice.

\subsection{Human studies}

1. Standard ELISA kit is able to detect MMP-9 from faecal samples.

2. Elevated faecal MMP-9 levels are present in patients with active UC compared to functional (IBS-D) patients or controls.

3. In UC patients faecal MMP-9 levels are excellent predictors of disease activity and show a significant correlation with the clinical and endoscopic scores.

4. Faecal MMP-9 levels correlate significantly with faecal calprotectin in UC.

5. Faecal MMP-9 can be used to distinguish CRC patients from patients with no polypoid lesions, with a high sensitivity and specificity.

6. With a lower cut-off level, faecal MMP-9 is able to identify nearly $60 \%$ of patients with a high-risk adenoma. 


\section{SUMMARY}

\section{Background}

Irritable bowel syndrome (IBS) is a functional gastrointestinal disorder characterized by continuous or remittent abdominal pain, bloating and altered bowel habits without recognized underlying organic aetiology. 5-20\% of patients seeking a gastroenterologist are diagnosed with IBS, causing considerably high medical and indirect costs. Despite its epidemiological significance, the pathophysiology of IBS is still not completely understood. Animal models, which reveal the pathophysiological background, could provide better treatments options for patients suffering from this disease. Impaired intestinal barrier function is one of the potential key steps of the development of IBS symptoms, therefore transgenic mice with constantly elevated intestinal permeability could be ideal animal model of the disease.

Clinically it is a great challenge to distinguish between functional and organic gastrointestinal diseases. Symptoms of ulcerative colitis (UC) in remission are often similar to those of IBS. Furthermore, colorectal cancer (CRC) is one of the leading malignancies worldwide, therefore cheap non-invasive screening methods are of great importance. Matrix metalloprotease 9 (MMP-9) is elevated in colonic biopsies, urine, and blood plasma of UC patients and its level is elevated in colonic tumour biopsies. So far, faecal MMP-9 levels have never been measured.

\section{$\underline{\text { Aims }}$}

The aims of the animal experiments in CAMLCKTg transgenic mice were: 1) to verify if the transgene has an effect on the gut permeability, animals' growth and intestinal transit, 2) to explore the effect of elevated intestinal permeability on the gut microbiota composition, 3) to study the moderately elevated gut permeability on behaviour and visceral sensitivity, 4) to investigate if the intestinal permeability elevation has an effect in the brain activity. In human studies our aims were: 1) to verify if the Standard ELISA Kit is able to detect MMP-9 from faecal samples 2) to compare faecal MMP-9 levels in UC patients to control subjects and a functional gastrointestinal disorder characterized by diarrhoea (IBS-D); 3) to test the 
correlation between UC disease activity and faecal levels of MMP-9; 4) to correlate faecal MMP-9 levels with a known faecal marker of UC activity, calprotectin. 5) to assess the faecal MMP-9 levels in patients undergoing total colonoscopy according to endoscopic and histological diagnosis.

\section{Methods}

Animal studies: To examine the permeability elevation on IBS-like symptoms, we used transgenic mice that specifically express Constitutively Active Myosin Light Chain Kinase (CAMLCKTg) in gut epithelium. Faecal microbiota composition, relative bacterial abundances were analysed using qPCR technique. Visceral sensitivity to colorectal distension (CRD) was determined in groups of wild type (WT) and CAMLCKTg female mice. Some groups were previously submitted to water avoidance stress, others treated with naloxone. Specificity of MLCK-driven modifications of visceral sensitivity was addressed using ML-7 as a MLCK inhibitor. Behavioural modifications were evaluated using the open-field test. Neuronal activity was examined using c-Fos immunostaining. Human investigations: Faecal MMP-9 and calprotectin levels were measured by enzyme-linked immunosorbent assay and lateral flow assay, respectively. To measure the efficacy of MMP-9 to detect UC or CRC, first we verified if the standard ELISA Kit is able to measure MMP9 from faecal samples. For the UC-study UC, IBS-D patients, and control subjects provided faecal samples for MMP-9 analysis. In UC patients, disease severity was evaluated by the Mayo score. In the CRC study 104 patients provided faecal samples for MMP-9 analysis. A total colonoscopy was performed; suspicious lesions were evaluated by histology. Based on the colonoscopic and histological results, patients were allocated to five groups: negative, diverticulosis, hyperplastic polyp, adenoma, and CRC.

\section{Key results}

Animal studies: CAMLCKTg animals have an altered faecal microbiota composition, similarly as observed in IBS. At the basal state, CAMLCKTg mice showed a visceral hyposensitivity compared with WT, which was abolished with ML7 as well as naloxone pretreatment. Upon stress stimulation, transgenic mice displayed a strong hypersensitivity to 
CRD. Submitted to the open-field, CAMLCKTg mice showed an anxiety-prone phenotype. CAMLCKTg mice have altered activity in certain brain regions compared to the wild type mice, what can be responsible to the behaviour modification. Human investigations: Level of MMP-9 was undetectable or very low in the faeces of all healthy controls and IBS-D patients. In UC patients, faecal MMP-9 levels significantly correlated with the overall Mayo score, endoscopic score, and the serum C-reactive protein level. Additionally, in UC patients faecal MMP-9 levels showed a significant correlation with a known disease activity marker, faecal calprotectin. In the CRC study faecal MMP-9 was significantly increased in CRC compared with all other groups. Faecal MMP-9 was suitable to distinguish CRC patients from controls.

\section{Conclusions \& Inferences}

Expression of CAMLCKTg within the mouse gut epithelium triggers increased permeability, which is associated with visceral hyposensitivity to CRD and anxiety. ML7 pretreatment abolished visceral hyposensitivity showing that visceral hyposensitivity is linked to MLCK. Moreover, inhibition induced by naloxone suggests the involvement of endogenous opioid pathways. Transgenic mice were also hyperresponsive to water avoidance stress. Dysbiosis is observed in the group of animals having CAMLCK transgene induced intestinal permeability elevation with an increased activity pattern in certain brain regions.

Faecal MMP-9 is a useful tool in the differential diagnosis of diarrheic disorders and in the non-invasive evaluation of disease activity and mucosal healing in UC. Faecal MMP-9 may be a promising new non-invasive marker in CRC. 


\section{ABBREVIATIONS}

AUC: area under the curve

CAMLCKTg: transgenic with constitutively active myosin light chain kinase

CD: Crohn’s disease

CDAI: Crohn’s Disease Activity Index

CRC: colorectal cancer

CRD: colorectal distension

CRP: C-reactive protein

ELISA: enzyme-linked immunosorbent assay

FIT: immunochemical faecal occult blood test

FITC: fluorescein isothiocyanate

FOBT: faecal occult blood test

gFOBT: guaiac faecal occult blood test

IBD: inflammatory bowel disease

IBS: irritable bowel syndrome

IBS-C: constipation predominant irritable bowel syndrome

IBS-M :mixed type irritable bowel syndrome

IBS-U: unclassified irritable bowel syndrome

IL : interleukin

EMG : electromyograph

MLCK : myosin light chain kinase 
MMP: matrix metalloprotease

ROC: receiver operating characteristic

ROI: region of interest

SD: standard deviation

SFRP2: secreted frizzled-related protein 2

TGF $\beta$ : transforming growth factor beta

TNF: tumour necrosis factor

TRITC: tetramethyl-rhodamine

UC: ulcerative colitis

WT: wild type 


\section{ACKNOWLEDGEMENTS}

I express my gratitude to Dr. Richard Róka, my tutor, without his support this work could not be done. I would like to express my gratitude to Prof. Vassilia Theodorou and Dr. Laurent Ferrier my tutors at INRA, who guided and supported my experimental work in Toulouse.

. I would like to express my deepest appreciation to Prof. János Lonovics, Prof. Tibor Wittmann, Prof. György Ábrahám, Prof. Csaba Lengyel the former and present heads of the First Dept. of Medicine for providing the conditions of experimental and clinical research in the department.

I am grateful to my collegue, Dr. Anita Annaházi for her enthusiastic help in both clinical and experimental studies.

I would like to thank my colleagues and co-authors Valerie Bacquié, Dr. Maïwenn Olier-Pierre, Dr. Marion Rincel, Dr. Belinda Ringot-Destrez, Sandrine Ellero-Simatos, Prof. Hélène Eutamène, Colette Bétoulières, Julie Thomas, Justin Lainé, Louise Gros, Mathilde Lévêque, Dr. Renaud Leonard, Cherryl Harkat, Dr. Catherine RobbeMasselot, Dr. Muriel Mercier-Bonin, Prof. Muriel Darnaudéry, Prof. Tamás Molnár, Dr. Klaudia Farkas, Dr. András Rosztóczy, Prof. Ferenc Izbéki, Dr. Krisztina Gecse, Prof. Ferenc Nagy, Dr. Imre Földesi, Mónika Szücs, Dr. Marta Dabek, Prof. Lionel Bueno, Dr. Szabolcs Ábrahám, Dr. Mariann Rutka, Klára Vadászi and Prof. György Lázár for their help.

Last but not least I would like to express my deepest gratitude to my family members who are always with me and support my work. 


\section{REFERENCES}

1. Drossman, D.A., Functional Gastrointestinal Disorders: History, Pathophysiology, Clinical Features and Rome IV. Gastroenterology, 2016.

2. Canavan, C., J. West, and T. Card, Review article: the economic impact of the irritable bowel syndrome. Aliment Pharmacol Ther, 2014. 40(9): p. 1023-34.

3. Scalera, A. and C. Loguercio, Focus on irritable bowel syndrome. Eur Rev Med Pharmacol Sci, 2012. 16(9): p. 1155-71.

4. Nicholl, B.I., et al., Psychosocial risk markers for new onset irritable bowel syndrome--results of a large prospective population-based study. Pain, 2008. 137(1): p. 147-55.

5. Pastorelli, L., et al., Central role of the gut epithelial barrier in the pathogenesis of chronic intestinal inflammation: lessons learned from animal models and human genetics. Front Immunol, 2013. 4: p. 280.

6. Gecse, K., et al., Leaky gut in patients with diarrhea-predominant irritable bowel syndrome and inactive ulcerative colitis. Digestion, 2012. 85(1): p. 40-6.

7. Ait-Belgnaoui, A., et al., Acute stress-induced hypersensitivity to colonic distension depends upon increase in paracellular permeability: role of myosin light chain kinase. Pain, 2005. 113(1-2): p. 141-7.

8. Marshall, J.K., et al., Intestinal permeability in patients with irritable bowel syndrome after a waterborne outbreak of acute gastroenteritis in Walkerton, Ontario. Aliment Pharmacol Ther, 2004. 20(11-12): p. 1317-22.

9. Zhou, Q., B. Zhang, and G.N. Verne, Intestinal membrane permeability and hypersensitivity in the irritable bowel syndrome. Pain, 2009. 146(1-2): p. 41-6.

10. Ferrier, L., et al., Stress-induced disruption of colonic epithelial barrier: role of interferongamma and myosin light chain kinase in mice. Gastroenterology, 2003. 125(3): p. 795-804.

11. Lauffer, A., et al., Subacute stress and chronic stress interact to decrease intestinal barrier function in rats. Stress, 2016. 19(2): p. 225-34.

12. Barbara, G., et al., New pathophysiological mechanisms in irritable bowel syndrome. Aliment Pharmacol Ther, 2004. 20 Suppl 2: p. 1-9.

13. Roka, R., et al., Dexamethasone prevents visceral hyperalgesia but not colonic permeability increase induced by luminal protease-activated receptor-2 agonist in rats. Gut, 2007. 56(8): p. 1072-8.

14. Vivinus-Nebot, M., et al., Combination of allergic factors can worsen diarrheic irritable bowel syndrome: role of barrier defects and mast cells. Am J Gastroenterol, 2012. 107(1): p. 75-81.

15. Su, L., et al., Targeted epithelial tight junction dysfunction causes immune activation and contributes to development of experimental colitis. Gastroenterology, 2009. 136(2): p. 55163.

16. Carroll, I.M., et al., Alterations in composition and diversity of the intestinal microbiota in patients with diarrhea-predominant irritable bowel syndrome. Neurogastroenterol Motil, 2012. 24(6): p. 521-30, e248.

17. Parkes, G.C., et al., Gastrointestinal microbiota in irritable bowel syndrome: their role in its pathogenesis and treatment. Am J Gastroenterol, 2008. 103(6): p. 1557-67.

18. Jalanka-Tuovinen, J., et al., Faecal microbiota composition and host-microbe cross-talk following gastroenteritis and in postinfectious irritable bowel syndrome. Gut, 2014. 63(11): p. 1737-45. 
19. Hellstrom, P.M., Pathophysiology of the irritable bowel syndrome - Reflections of today. Best Pract Res Clin Gastroenterol, 2019. 40-41: p. 101620.

20. Vermeire, S., G. Van Assche, and P. Rutgeerts, Laboratory markers in IBD: useful, magic, or unnecessary toys? Gut, 2006. 55(3): p. 426-31.

21. Saverymuttu, S.H., et al., Differing acute phase responses in Crohn's disease and ulcerative colitis. Gut, 1986. 27(7): p. 809-13.

22. Poullis, A.P., et al., A new, highly sensitive assay for C-reactive protein can aid the differentiation of inflammatory bowel disorders from constipation- and diarrhoeapredominant functional bowel disorders. Eur J Gastroenterol Hepatol, 2002. 14(4): p. 409-12.

23. Shine, B., et al., C-reactive protein as an aid in the differentiation of functional and inflammatory bowel disorders. Clin Chim Acta, 1985. 148(2): p. 105-9.

24. Schoepfer, A.M., et al., Discriminating IBD from IBS: comparison of the test performance of fecal markers, blood leukocytes, CRP, and IBD antibodies. Inflamm Bowel Dis, 2008. 14(1): p. 32-9.

25. Kucharzik, T., et al., Recent understanding of IBD pathogenesis: implications for future therapies. Inflamm Bowel Dis, 2006. 12(11): p. 1068-83.

26. Kucharzik, T., et al., Neutrophil transmigration in inflammatory bowel disease is associated with differential expression of epithelial intercellular junction proteins. Am J Pathol, 2001. 159(6): p. 2001-9.

27. Saverymuttu, S.H., V.S. Chadwick, and H.J. Hodgson, Granulocyte migration in ulcerative colitis. Eur J Clin Invest, 1985. 15(2): p. 60-3.

28. Dabek, M., et al., Luminal cathepsin $g$ and protease-activated receptor 4: a duet involved in alterations of the colonic epithelial barrier in ulcerative colitis. Am J Pathol, 2009. 175(1): p. 207-14.

29. Roseth, A.G., et al., Assessment of the neutrophil dominating protein calprotectin in feces. A methodologic study. Scand J Gastroenterol, 1992. 27(9): p. 793-8.

30. Roseth, A.G., et al., Assessment of disease activity in ulcerative colitis by faecal calprotectin, a novel granulocyte marker protein. Digestion, 1997. 58(2): p. 176-80.

31. Costa, F., et al., Role of faecal calprotectin as non-invasive marker of intestinal inflammation. Dig Liver Dis, 2003. 35(9): p. 642-7.

32. Tibble, J., et al., A simple method for assessing intestinal inflammation in Crohn's disease. Gut, 2000. 47(4): p. 506-13.

33. Baugh, M.D., et al., Matrix metalloproteinase levels are elevated in inflammatory bowel disease. Gastroenterology, 1999. 117(4): p. 814-22.

34. Manfredi, M.A., et al., Increased incidence of urinary matrix metalloproteinases as predictors of disease in pediatric patients with inflammatory bowel disease. Inflamm Bowel Dis, 2008. 14(8): p. 1091-6.

35. Sung, J.J., et al., Asia Pacific consensus recommendations for colorectal cancer screening. Gut, 2008. 57(8): p. 1166-76.

36. Siegel, R., D. Naishadham, and A. Jemal, Cancer statistics, 2012. CA Cancer J Clin, 2012. 62(1): p. 10-29.

37. Ferlay, J., et al., Cancer incidence and mortality patterns in Europe: estimates for 40 countries in 2012. Eur J Cancer, 2013. 49(6): p. 1374-403.

38. Binefa, G., et al., Colorectal cancer: from prevention to personalized medicine. World J Gastroenterol, 2014. 20(22): p. 6786-808.

39. Konrad, G., Dietary interventions for fecal occult blood test screening: systematic review of the literature. Can Fam Physician, 2010. 56(3): p. 229-38. 
40. European Colorectal Cancer Screening Guidelines Working, G., et al., European guidelines for quality assurance in colorectal cancer screening and diagnosis: overview and introduction to the full supplement publication. Endoscopy, 2013. 45(1): p. 51-59.

41. Unsal, D., et al., Gelatinase B expression as a prognostic factor in patients with stage II/III rectal carcinoma treated by postoperative adjuvant therapy. Am J Clin Oncol, 2008. 31(1): $p$. 55-63.

42. Araujo, R.F., Jr., et al., Prognostic and diagnostic implications of MMP-2, MMP-9, and VEGFalpha expressions in colorectal cancer. Pathol Res Pract, 2015. 211(1): p. 71-7.

43. Yang, B., et al., Matrix metalloproteinase-9 overexpression is closely related to poor prognosis in patients with colon cancer. World J Surg Oncol, 2014. 12: p. 24.

44. Bergstrom, A., et al., Introducing GUt low-density array (GULDA): a validated approach for qPCR-based intestinal microbial community analysis. FEMS Microbiol Lett, 2012. 337(1): p. 38-47.

45. Bergstrom, A., et al., Establishment of intestinal microbiota during early life: a longitudinal, explorative study of a large cohort of Danish infants. Appl Environ Microbiol, 2014. 80(9): p. 2889-900.

46. Riba, A., et al., Paneth Cell Defects Induce Microbiota Dysbiosis in Mice and Promote Visceral Hypersensitivity. Gastroenterology, 2017. 153(6): p. 1594-1606.e2.

47. Blair, S.A., et al., Epithelial myosin light chain kinase expression and activity are upregulated in inflammatory bowel disease. Lab Invest, 2006. 86(2): p. 191-201.

48. Van den Abbeele, P., et al., Arabinoxylo-Oligosaccharides and Inulin Impact Inter-Individual Variation on Microbial Metabolism and Composition, Which Immunomodulates Human Cells. J Agric Food Chem, 2018. 66(5): p. 1121-1130.

49. Owen, C.D., et al., Unravelling the specificity and mechanism of sialic acid recognition by the gut symbiont Ruminococcus gnavus. Nature Communications, 2017. 8(1): p. 2196.

50. Earley, H., et al., A Preliminary Study Examining the Binding Capacity of Akkermansia muciniphila and Desulfovibrio spp., to Colonic Mucin in Health and Ulcerative Colitis. PLOS ONE, 2015. 10(10): p. e0135280.

51. Jeffery, I.B., et al., An irritable bowel syndrome subtype defined by species-specific alterations in faecal microbiota. Gut, 2012. 61(7): p. 997.

52. Ford, A.C. and N.J. Talley, Mucosal inflammation as a potential etiological factor in irritable bowel syndrome: a systematic review. J Gastroenterol, 2011. 46(4): p. 421-31.

53. Piche, T., et al., Impaired intestinal barrier integrity in the colon of patients with irritable bowel syndrome: involvement of soluble mediators. Gut, 2009. 58(2): p. 196-201.

54. Annahazi, A., et al., Luminal cysteine-proteases degrade colonic tight junction structure and are responsible for abdominal pain in constipation-predominant IBS. Am J Gastroenterol, 2013. 108(8): p. 1322-31.

55. Martinez, C., et al., Diarrhoea-predominant irritable bowel syndrome: an organic disorder with structural abnormalities in the jejunal epithelial barrier. Gut, 2013. 62(8): p. 1160-8.

56. Ait-Belgnaoui, A., et al., Lactobacillus farciminis treatment suppresses stress induced visceral hypersensitivity: a possible action through interaction with epithelial cell cytoskeleton contraction. Gut, 2006. 55(8): p. 1090-4.

57. Wilder-Smith, C.H., et al., Brain functional magnetic resonance imaging of rectal pain and activation of endogenous inhibitory mechanisms in irritable bowel syndrome patient subgroups and healthy controls. Gut, 2004. 53(11): p. 1595-601.

58. Larauche, M., et al., Visceral analgesia induced by acute and repeated water avoidance stress in rats: sex difference in opioid involvement. Neurogastroenterol Motil, 2012. 24(11): p. 1031e547. 
59. Ren, S., et al., The paraventricular thalamus is a critical thalamic area for wakefulness. Science, 2018. 362(6413): p. 429-434.

60. Careaga, M.B.L., C.E.N. Girardi, and D. Suchecki, Variability in response to severe stress: highly reactive rats exhibit changes in fear and anxiety-like behavior related to distinct neuronal co-activation patterns. Behav Brain Res, 2019. 373: p. 112078.

61. Opdenakker, G., P.E. Van den Steen, and J. Van Damme, Gelatinase B: a tuner and amplifier of immune functions. Trends Immunol, 2001. 22(10): p. 571-9.

62. Castaneda, F.E., et al., Targeted deletion of metalloproteinase 9 attenuates experimental colitis in mice: central role of epithelial-derived MMP. Gastroenterology, 2005. 129(6): p. 1991-2008.

63. Nakajima, M., et al., Serum and plasma $M(r) 92,000$ progelatinase levels correlate with spontaneous metastasis of rat 13762NF mammary adenocarcinoma. Cancer Res, 1993. 53(23): p. 5802-7.

64. Chan, L.W., et al., Urinary VEGF and MMP levels as predictive markers of 1-year progressionfree survival in cancer patients treated with radiation therapy: a longitudinal study of protein kinetics throughout tumor progression and therapy. J Clin Oncol, 2004. 22(3): p. 499-506.

65. Fernandez, C.A., et al., The matrix metalloproteinase-9/neutrophil gelatinase-associated lipocalin complex plays a role in breast tumor growth and is present in the urine of breast cancer patients. Clin Cancer Res, 2005. 11(15): p. 5390-5.

66. Moses, M.A., et al., Increased incidence of matrix metalloproteinases in urine of cancer patients. Cancer Res, 1998. 58(7): p. 1395-9.

67. Smith, E.R., et al., Urinary biomarkers predict brain tumor presence and response to therapy. Clin Cancer Res, 2008. 14(8): p. 2378-86.

68. Lakatos, G., et al., The behavior of matrix metalloproteinase-9 in lymphocytic colitis, collagenous colitis and ulcerative colitis. Pathol Oncol Res, 2012. 18(1): p. 85-91.

69. Kossakowska, A.E., et al., Elevated plasma gelatinase A (MMP-2) activity is associated with quiescent Crohn's Disease. Ann N Y Acad Sci, 1999. 878: p. 578-80.

70. Gaya, D.R., et al., Faecal calprotectin in the assessment of Crohn's disease activity. Qjm, 2005. 98(6): p. 435-41.

71. Jones, J., et al., Relationships between disease activity and serum and fecal biomarkers in patients with Crohn's disease. Clin Gastroenterol Hepatol, 2008. 6(11): p. 1218-24.

72. Poullis, A., et al., Emerging role of calprotectin in gastroenterology. J Gastroenterol Hepatol, 2003. 18(7): p. 756-62.

73. Hyuga, S., et al., Autocrine factor enhancing the secretion of $M(r)$ 95,000 gelatinase (matrix metalloproteinase 9) in serum-free medium conditioned with murine metastatic colon carcinoma cells. Cancer Res, 1994. 54(13): p. 3611-6.

74. Deryugina, E.I., et al., Tissue-infiltrating neutrophils constitute the major in vivo source of angiogenesis-inducing MMP-9 in the tumor microenvironment. Neoplasia, 2014. 16(10): p. 771-88.

75. Provatopoulou, X., et al., Circulating levels of matrix metalloproteinase-9 (MMP-9), neutrophil gelatinase-associated lipocalin (NGAL) and their complex MMP-9/NGAL in breast cancer disease. BMC Cancer, 2009. 9: p. 390.

76. Ylisirnio, S., M. Hoyhtya, and T. Turpeenniemi-Hujanen, Serum matrix metalloproteinases -2, 9 and tissue inhibitors of metalloproteinases $-1,-2$ in lung cancer--TIMP-1 as a prognostic marker. Anticancer Res, 2000. 20(2b): p. 1311-6.

77. Hurst, N.G., et al., Elevated serum matrix metalloproteinase 9 (MMP-9) concentration predicts the presence of colorectal neoplasia in symptomatic patients. Br J Cancer, 2007. 97(7): p. 971-7. 
78. Mroczko, B., et al., The diagnostic value of matrix metalloproteinase 9 (MMP-9) and tissue inhibitor of matrix metalloproteinases 1 (TIMP-1) determination in the sera of colorectal adenoma and cancer patients. Int J Colorectal Dis, 2010. 25(10): p. 1177-84.

79. Duvillard, L., et al., A case-control study of pre-operative levels of serum neutrophil gelatinase-associated lipocalin and other potential inflammatory markers in colorectal cancer. BMC Cancer, 2014. 14: p. 912.

80. Kanthan, R., J.L. Senger, and S.C. Kanthan, Fecal molecular markers for colorectal cancer screening. Gastroenterol Res Pract, 2012. 2012: p. 184343.

81. Lansdorp-Vogelaar, I., et al., Stool DNA testing to screen for colorectal cancer in the Medicare population: a cost-effectiveness analysis. Ann Intern Med, 2010. 153(6): p. 368-77.

82. Morikawa, T., et al., A comparison of the immunochemical fecal occult blood test and total colonoscopy in the asymptomatic population. Gastroenterology, 2005. 129(2): p. 422-8.

83. Haug, U., S. Hundt, and H. Brenner, Quantitative immunochemical fecal occult blood testing for colorectal adenoma detection: evaluation in the target population of screening and comparison with qualitative tests. Am J Gastroenterol, 2010. 105(3): p. 682-90.

84. Mak, T., et al., Molecular stool screening for colorectal cancer. Br J Surg, 2004. 91(7): p. 790800.

85. Tagore, K.S., T.R. Levin, and M.J. Lawson, The evolution to stool DNA testing for colorectal cancer. Aliment Pharmacol Ther, 2004. 19(12): p. 1225-33.

86. Huang, Z., L. Li, and J. Wang, Hypermethylation of SFRP2 as a potential marker for stoolbased detection of colorectal cancer and precancerous lesions. Dig Dis Sci, 2007. 52(9): p. 2287-91.

87. Baek, Y.H., et al., Stool methylation-specific polymerase chain reaction assay for the detection of colorectal neoplasia in Korean patients. Dis Colon Rectum, 2009. 52(8): p. 1452-9; discussion 1459-63.

88. Haug, U., S. Hundt, and H. Brenner, Sensitivity and specificity of faecal tumour M2 pyruvate kinase for detection of colorectal adenomas in a large screening study. Br J Cancer, 2008. 99(1): p. 133-5.

89. Odabasi, M., et al., Role of human neutrophil gelatinase associated lipocalin (NGAL) and Matrix Metalloproteinase-9 (MMP-9) overexpression in neoplastic colon polyps. Int J Clin Exp Med, 2014. 7(9): p. 2804-11.

90. Herszenyi, L., et al., Matrix metalloproteinase-9 expression in the normal mucosa-adenomadysplasia-adenocarcinoma sequence of the colon. Pathol Oncol Res, 2008. 14(1): p. 31-7. 
ANNEXES 


\section{Journal Pre-proof}

Targeted intestinal tight junction hyperpermeability alters the microbiome, behavior, and visceromotor responses

Orsolya Inczefi, Valérie Bacquié, Maïwenn Olier-Pierre, Marion Rincel, Belinda Ringot-Destrez, Sandrine Ellero-Simatos, Hélène Eutamène, Colette Bétoulières, Julie Thomas, Justin Laine, Louise Gros, Mathilde Lévêque, Renaud Leonard, Cherryl Harkat, Catherine Robbe-Masselot, Richard Roka, Muriel Mercier-Bonin, Vassilia Theodorou, Muriel Darnaudéry, Jerrold R. Turner, Laurent Ferrier

PII: S2352-345X(20)30035-7

DOI: $\quad$ https://doi.org/10.1016/j.jcmgh.2020.02.008

Reference: JCMGH 588

To appear in: Cellular and Molecular Gastroenterology and Hepatology Accepted Date: 27 February 2020

Please cite this article as: Inczefi O, Bacquié V, Olier-Pierre M, Rincel M, Ringot-Destrez B, ElleroSimatos $S$, Eutamène $H$, Bétoulières $C$, Thomas J, Laine J, Gros L, Lévêque $M$, Leonard R, Harkat C, Robbe-Masselot C, Roka R, Mercier-Bonin M, Theodorou V, Darnaudéry M, Turner JR, Ferrier L, Targeted intestinal tight junction hyperpermeability alters the microbiome, behavior, and visceromotor responses, Cellular and Molecular Gastroenterology and Hepatology (2020), doi: https://doi.org/10.1016/ j.jcmgh.2020.02.008.

This is a PDF file of an article that has undergone enhancements after acceptance, such as the addition of a cover page and metadata, and formatting for readability, but it is not yet the definitive version of record. This version will undergo additional copyediting, typesetting and review before it is published in its final form, but we are providing this version to give early visibility of the article. Please note that, during the production process, errors may be discovered which could affect the content, and all legal disclaimers that apply to the journal pertain.

(C) 2020 The Authors. Published by Elsevier Inc. on behalf of the AGA Institute. 
Targeted intestinal tight junction hyperpermeability alters the microbiome, behavior, and visceromotor responses

Orsolya Inczefi ${ }^{1,2,}$, Valérie Bacquié ${ }^{1, *}$, Maïwenn Olier-Pierre ${ }^{1}$, Marion Rincel ${ }^{3}$, Belinda RingotDestrez $^{4}$, Sandrine Ellero-Simatos ${ }^{1}$, Hélène Eutamène ${ }^{1}$, Colette Bétoulières ${ }^{1}$, Julie Thomas ${ }^{3}$, Justin Laine ${ }^{3}$, Louise Gros $^{3}$, Mathilde Lévêque ${ }^{1}$, Renaud Leonard ${ }^{4}$, Cherryl Harkat ${ }^{1}$, Catherine Robbe-Masselot ${ }^{4}$, Richard Roka ${ }^{2}$, Muriel Mercier-Bonin ${ }^{1}$, Vassilia Theodorou', Muriel Darnaudéry ${ }^{3}$, Jerrold R Turner ${ }^{5 \S}$, and Laurent Ferrier ${ }^{1 \S}$

${ }^{1}$ INRAE, UMR 1331 ToxAlim, Toulouse, France

${ }^{2}$ First Department of Medicine, University of Szeged, Szeged, Hungary

${ }^{3}$ University of Bordeaux, INRAE, Nutrition and Integrative Neurobiology, UMR 1286, 33076

Bordeaux, France

${ }^{4}$ Université de Lille, Unité de Glycobiologie Structurale et Fonctionnelle, Villeneuve d'Ascq, France

${ }^{5}$ Laboratory of Mucosal Barrier Pathobiology, Department of Pathology, Brigham and Women's Hospital, Harvard Medical School, Boston, Massachusetts, USA

* These authors contributed equally

${ }^{\S}$ Corresponding authors:

Laurent Ferrier, PhD

INRA, UMR 1331 ToxAlim, Group of Neuro-Gastroenterology \& Nutrition, Toulouse, France Present address: Nestlé Research, Institute of Health Sciences, Department of Gastro-Intestinal Health

Route du Jorat 57 - Vers-chez-les-Blanc

1000 Lausanne 26

Switzerland

Phone: +4121785 8421

E-mail: laurent.ferrier@rd.nestle.com

Jerrold R. Turner, MD, PhD

77 Avenue Louis Pasteur

NRB 730

Boston, MA 02115

E-mail: jrturner@bwh.harvard.edu

LENGTH: 999 words

RUNNING HEADER: Diverse responses to intestinal hyperpermeability 
CONFLICTS OF INTEREST: JRT is a cofounder of Thelium Therapeutics. LF is currently an employee of Nestlé Research (Société des Produits Nestlé S.A.).

CONTRIBUTIONS: Conceptualization: MOP, MMB, MD, VT,JRT, LF; Experimentation: OI, VB, MOP, BRD, MR, SES, CB, ML, JT, JL, LG, RL, CH; Data analysis: OI, VB, MOP, HE, CRM, MR, RR, MMB, MD, JRT, LF; Manuscript preparation and revision: MD, JRT, LF.

KEYWORDS: myosin light chain kinase, MLCK, tight junction, microbiome, stress

ACKNOWLEDGEMENTS: This work was supported by an institutional grant from INRA and by NIH grants R01DK61931 and R01DK68271 to JRT. MD was supported by Bordeaux University, by the FFAS (Fond Français Alimentation Santé), and the ANR (Agence Nationale de la Recherche). MR was supported by the French ministry of research and education and Labex Brain. JT was a recipient of a fellowship from the French Society of Paediatric Research. OI was a recipient of a fellowship from the Nutrition, Chemical Food Safety and Consumer Behaviour Division of INRA. 
Markedly increased intestinal permeability occurs in inflammatory bowel disease (IBD), graftversus-host disease (GVHD), celiac disease, and multiple organ dysfunction. In these diseases, effectors of increased permeability include immune signaling, ${ }^{1}$ microbiome, ${ }^{2}$ and corticosteroids $^{3}$ that, in part, signal through epithelial myosin light chain kinase (MLCK). More modest permeability increases occur in other disorders, including irritable bowel syndrome (IBS), autism spectrum disorder (ASD), depression, and stress-related disorders. Data directly linking barrier loss to disease phenotypes, however, are lacking.

To define the impact of modestly-increased intestinal permeability, we studied transgenic mice with intestinal epithelial-specific constitutively-active myosin light chain kinase (CAMLCK) expression. This MLCK-dependent tight junction regulation increased intestinal permeability (Fig. S1A,B). ${ }^{1}$ Nevertheless, postnatal growth (Fig. S1C), reproduction, intestinal transit (Fig. S1D), and intestinal histology, epithelial proliferation (a sensitive indicator of epithelial damage), and epithelial turnover are unaffected in CAMLCK transgenic $\left(C A M L C K^{T g}\right)$ mice. ${ }^{1}$ In contrast, mucosal tumor necrosis factor- $\alpha$, interferon- $\gamma$, IL-10, and IL-13 transcripts as well as numbers of lamina propria neutrophils, CD4 ${ }^{+} \mathrm{T}$ cells, and $\lg \mathrm{A}^{+}$plasma cells are modestly increased by CAMLCK expression. ${ }^{1,2}$ Subclinical inflammation is, therefore, present and, by microbiomedependent, IL-17-mediated processes, affords partial protection from acute pathogen invasion. ${ }^{2}$ Immune activation is nevertheless unlikely to amplify CAMLCK-driven permeability increases, as barrier function and ZO-1 anchoring are both acutely normalized by enzymatic MLCK inhibition. $^{1,4}$

We initially analyzed the gut microbiome of $31 \mathrm{WT}$ and $C A M L C K^{T g}$ pups born to $8 \mathrm{WT}$ dams. The microbiomes segregated by pup genotype but not dam (Fig. S1E) and included increased Clostridium and decreased Bacteroidetes, Enterococcus spp, and Prevotella in CAMLCK ${ }^{T g}$ mice (Fig. S1F). Increased intestinal permeability can therefore cause dysbiosis-like microbiome shifts. Interestingly, maternal separation, which increases intestinal permeability, causes similar alterations and can be partially corrected by MLCK inhibitor-induced barrier restoration. ${ }^{5}$

Microbiome alterations overlapping with the above have been reported in IBS and ASD. We therefore asked if $C A M L C K^{T g}$ mice displayed anxiety-like behavior, as occurs in those disorders, using the open-field test (Fig. 1C). Both the percentage of distance traveled in the center and the fraction of time spent in the center of the open field were reduced in $C A M L C K^{T g}$ mice (Fig. 1C); this did not reflect reduced locomotor activity, as total distance traveled in the entire area was similar in CAMLCK ${ }^{T g}$ and WT mice (Fig. 1C). These data are consistent with increased anxiety-like behavior in $C A M L C K^{T g}$ mice. Although the results cannot differentiate between 
direct effects of increased permeability and those requiring intermediate mediators, these data demonstrate that intestinal permeability increases can influence behavior.

Stress and increased permeability have been associated with enhanced visceral sensitivity in humans and rodents. Surprisingly, $C A M L C K^{T g}$ mice displayed striking visceral analgesia to colorectal distension relative to WT littermates (Fig. 1D). Sensitivity was restored by enzymatic MLCK inhibition, water avoidance stress, or naloxone-mediated opioid receptor antagonism (Fig. 1D). Although this effect of increased permeability on visceral sensitivity was unexpected, it is remarkably similar to the naloxone-reversible visceral analgesia reported in chronicallystressed female rats ${ }^{6}$ and naloxone-sensitive inhibition of nociceptive neurons by supernatants of colitic human and murine tissues. ${ }^{7}$

Studies of female IBS patients have linked increased permeability to altered functional and structural brain connectivity. ${ }^{8}$ Thus, although responses to colorectal distension can be mediated by spinal reflexes and sensory, limbic, and paralimbic regions of the brain, ${ }^{9}$ we asked if neuronal activation was modified by CAMLCK-induced permeability increases. C-Fos immunolabeling, an indicator of neuronal activity, was significantly greater in the paraventricular nucleus of the thalamus, the paraventricular nucleus of the hypothalamus, and the hippocampus, but not the medial prefrontal cortex, nucleus accumbens, or amygdala, of $C A M L C K^{T g}$, relative to WT, mice (Figs. 2, S2). Increased intestinal permeability may therefore increase basal neuronal activity in areas of the brain that regulate responses to visceral pain or stress $^{9}$ but not those associated with conscious visceral sensation.

These results demonstrate that increased intestinal permeability can impact i) gut microbiome composition; ii) behavior; iii) visceral pain responses; and iv) neuronal activation within the brain. Critically, these changes are all results, rather than causes, of intestinal barrier loss, as the latter was induced by targeted CAMLCK expression.

The sites of neuronal activation in $C A M L C K^{T g}$ mice support the hypothesis that increased intestinal permeability can activate the hypothalamic-pituitary-adrenal axis. ${ }^{10}$ Conversely, hypothalamic-pituitary-adrenal axis activation by exogenous stress can induce intestinal permeability increases. ${ }^{3}$ Thus, as has been proposed in IBD and GVHD, a self-amplifying cycle may ultimately direct the diverse phenotypes induced by MLCK-dependent, intestinal permeability increases. Further study is needed to define the complex relationships between intestinal permeability, stress, behavioral alterations, visceromotor responses, microbiome composition, and other abnormalities. 
66 These data are the first to assess behavior in a model where a targeted increase in intestinal

67 tight junction permeability is the only direct perturbation. The results demonstrate,

68 unequivocally, that modest tight junction permeability increases induced via a physiologically-

69 and pathophysiologically-relevant mechanism are sufficient to trigger local and systemic

70 microbial, behavioral, and neurosensory changes. This provides new perspective with which to

71 understand previously hypothesized cause-effect relationships that have been proposed on the 72 basis of correlative data. 


\section{Figure legends:}

Figure 1: Increased intestinal permeability modifies behavior and visceral sensitivity. A. Videotracking paths of representative WT and $C A M L C K^{T g}$ mice in the open field test. Percent distance traveled in the center (dashed lines), percent time in the center, and overall distance traveled in the entire field are shown. $C A M L C K^{T g}$ (blue circles, $n=8$ ) and WT (red squares, $n=9$ ) littermates were tested. mean \pm SEM. ${ }^{*}, p<0.05 ;{ }^{* *}, p<0.01$, Mann-Whitney U test. B. Stepwise colorectal distension-induced visceromotor responses in $C A M L C K^{T g}$ (blue circles, $n=7$ ) were reduced relative to WT (red squares, $n=7$ ) littermates. Genotype-specific differences were eliminated by MLCK inhibition, water avoidance stress, or naloxone treatment. $n=5-9$ per condition; for each treatment (vehicle control CAMLCK ${ }^{T g}$ and WT mice from the same experiment are shown with pale symbols in the last three graphs). mean $\pm S E M ;{ }^{* *}, p<0.01,2-$ way ANOVA.

Figure 2: Increased intestinal permeability induces increased C-Fos immunolabelling in selected brain regions. CAMLCK ${ }^{T g}$ (blue circles, $n=5-6$ ) and WT (red squares, $n=5-6$ ) littermates. Representative images of C-Fos immunolabeled brains from $C A M L C K^{T g}$ and WT mice. Bars $=200 \mu \mathrm{m}$; mean $\pm S E M ;{ }^{*}, p<0.05$, t-test. 


\section{References}

91 1. Su L, et al. Gastroenterology 2009;136:551-63.

92 2. Edelblum KL, et al. Cell Mol Gastroenterol Hepatol 2017;4:285-297.

93 3. Meddings JB, et al. Gastroenterology 2000;119:1019-28.

94 4. Yu D, et al. Proc Natl Acad Sci U S A 2010;107:8237-41.

95 5. Rincel M, et al. Psychopharmacology (Berl) 2019;236:1583-1596.

96 6. Larauche M, et al. Neurogastroenterol Motil 2012;24:1031-e547.

97 7. Guerrero-Alba R, et al. Gut 2017;66:2121-2131.

98 8. Witt ST, et al. Neuroimage Clin 2019;21:101602.

99 9. Larauche M, et al. Neurogastroenterol Motil 2019;31:e13489.

100 10. Ait-Belgnaoui A, et al. Psychoneuroendocrinology 2012;37:1885-95. 


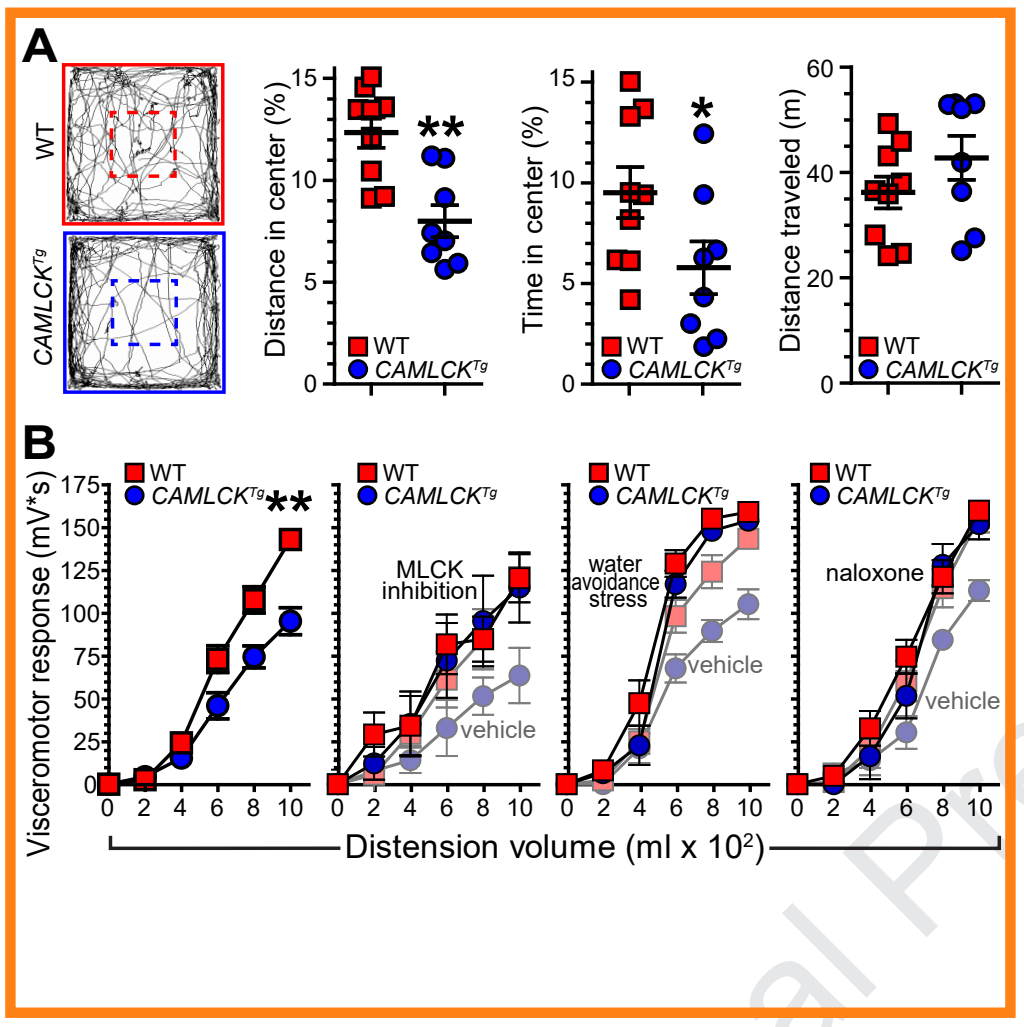




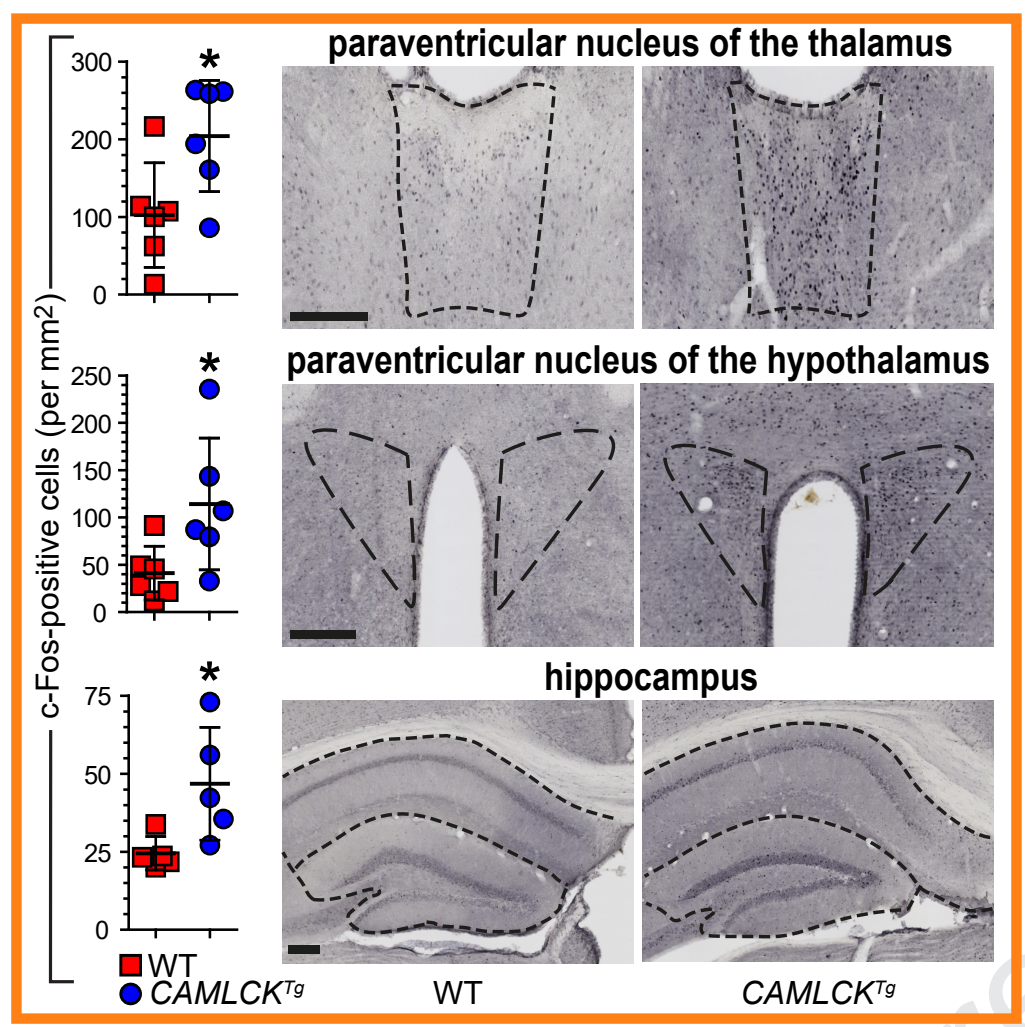




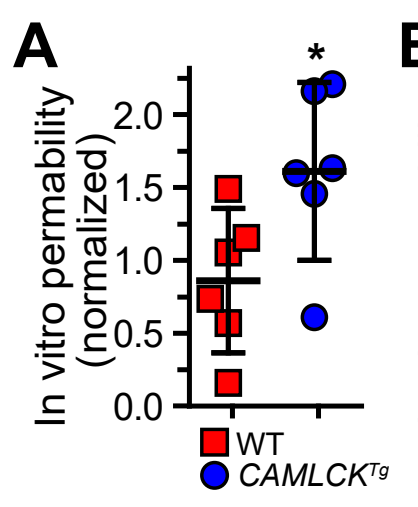

E

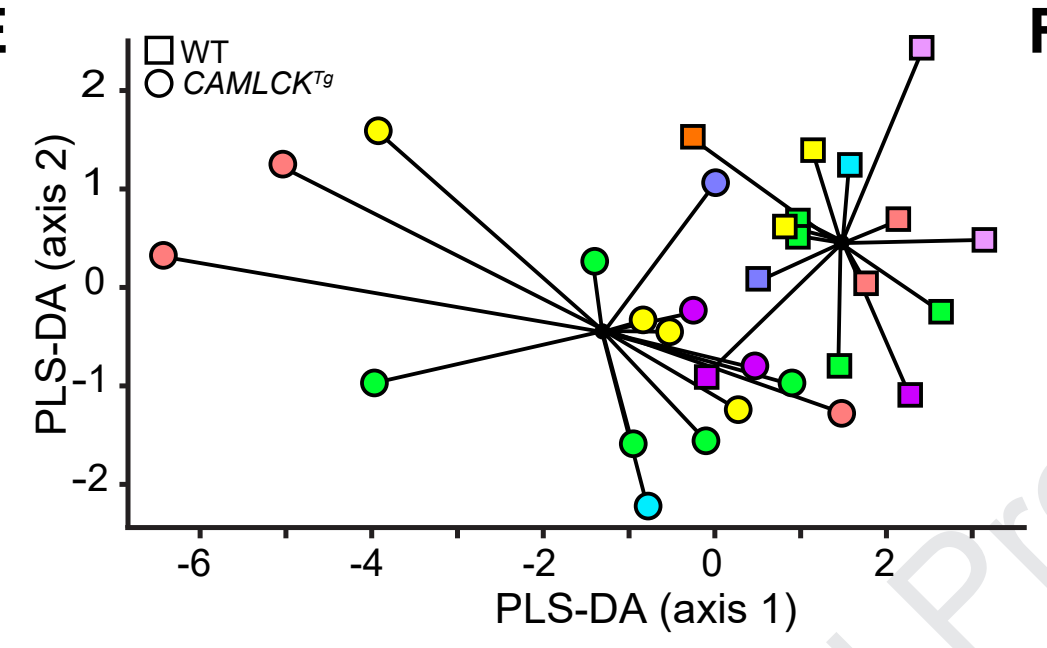

C

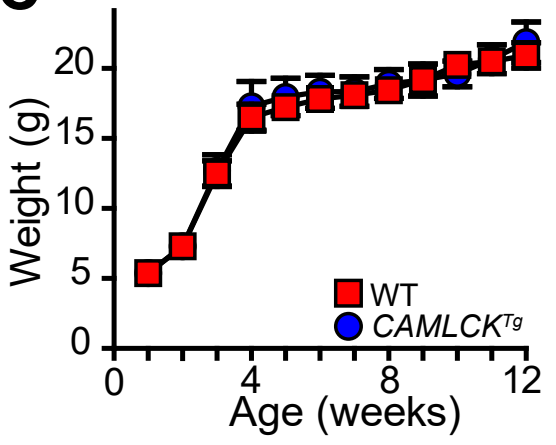

D

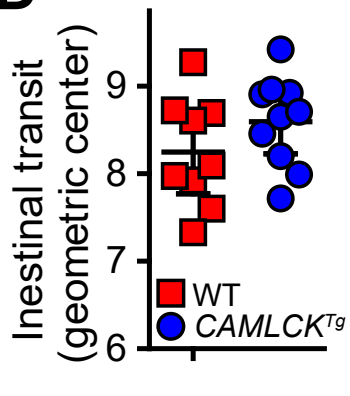

$\mathbf{F}$

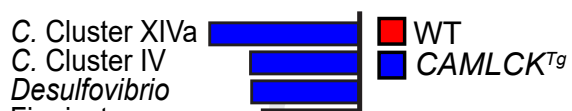

Desulfovibrio

Firmicutes

C. butyricum

$R$. gnavus

Enterobacteriaceae

Prevotella

B. fragilis

M. smithii

Bacteroides

B. thetaiotaomicron

Bifidobacterium

E. hallii

Bacteroides/Prevotella

Enterococcus

Bacteroidetes

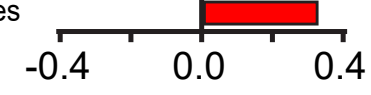




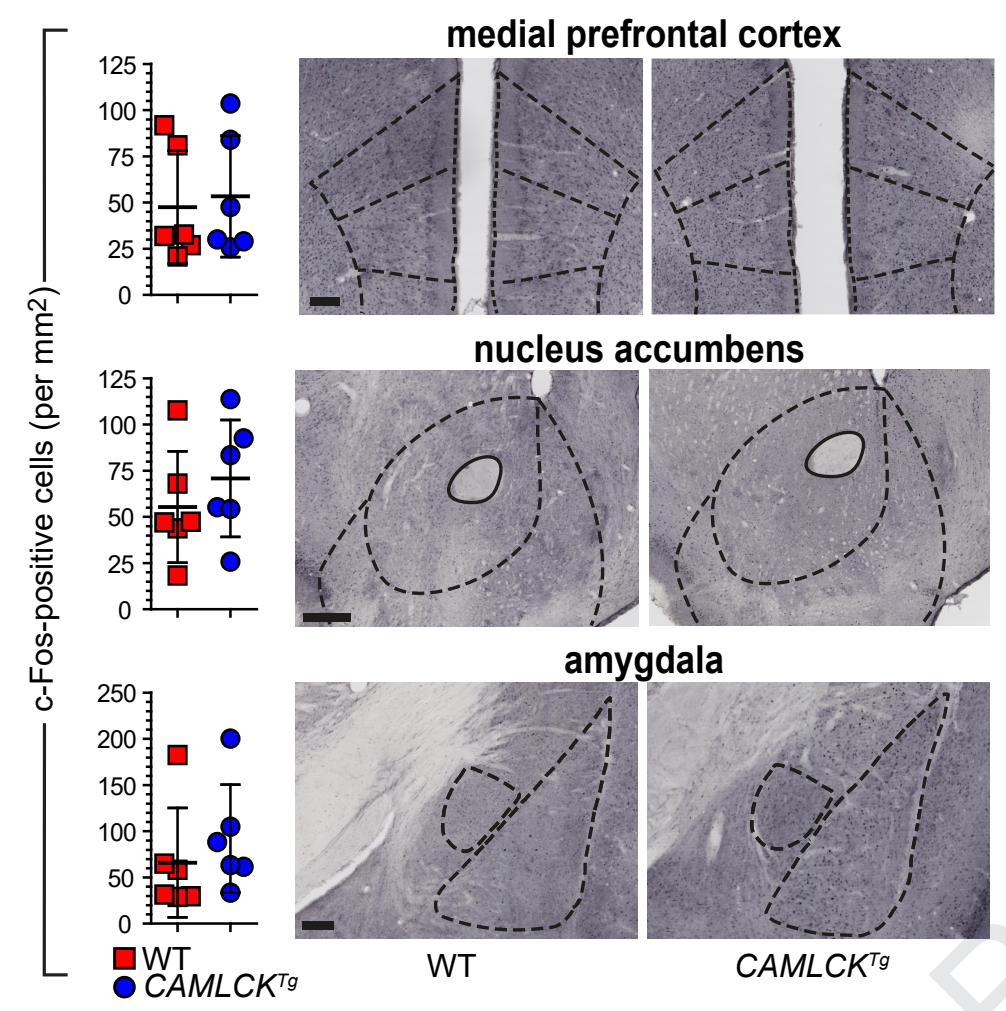




\section{Supplemental Figure 1:}

A. Trans-jejunal fluorescein flux was increased in $C A M L C K^{T g}$ (blue circles) relative to WT (red squares) littermates. mean $\pm S D ;{ }^{*}, p<0.05$, Mann-Whitney $U$ test.

B. In vivo analysis using FITC-4kDa dextran demonstrated increased permeability of CAMLCK ${ }^{T g}$ (blue circles, $n=19$ ) relative to WT (red squares, $n=20$ ) littermates. mean $\pm S D ;{ }^{*}, p<0.05$, t-test.

C. Weight gain was similar in WT (red squares, $\mathrm{n}=6$ ) and $C A M L C K^{T g}$ (blue circles, $\mathrm{n}=6$ ) littermates. mean $\pm S D$.

D. Intestinal transit was similar in WT (red squares, $\mathrm{n}=10$ ) and $C A M L C K^{T g}$ (blue circles, $\mathrm{n}=9$ ) littermates. mean $\pm S D$.

E. Partial least squares discriminant analysis (PLS-DA) score plot based on the relative abundances of 18 microbial taxa in gut contents of $C A M L C K^{T g}$ (circles, $\mathrm{n}=16$ ) and WT (squares, $\mathrm{n}=15$ ) born to 8 different dams (each color represents one dam).

F. Relative abundances of microbial communities in $C A M L C K^{T g}$ (blue) and WT (red) mice. Diagrams indicate regions analyzed. 


\section{Supplemental Figure 2:}

CAMLCK ${ }^{T g}$ (blue circles, $\mathrm{n}=5-6$ ) and WT (red squares, $\mathrm{n}=5-6$ ) littermates. Representative images of C-Fos immunolabeled brains from CAMLCK ${ }^{T g}$ and WT mice. Bars $=200 \mu \mathrm{m}$; mean \pm SEM; *,$p<0.05$, t-test. 


\section{Supplemental Methods}

\section{Animals}

CAMLCK ${ }^{T g}$ mice $^{1-4}(\mathrm{Tg}(\mathrm{Vil}-\mathrm{FLAG}-\mathrm{CAMLCK}) \# \mathrm{Jrt})$ were maintained as male heterozygotes on C57BL/6J background. These were mated with WT C57BL/6J females to produce WT and $C A M L C K^{T g}$ littermates. At weaning, female mice were separated and housed at constant temperature $\left(22 \pm 1^{\circ} \mathrm{C}\right)$ with a 12 hour light/dark cycle. Food (Teklad 2018, Envigo) and water were available ad libitum. All experiments were performed at 8 weeks of age. Procedures were approved by the Ethical Committee CEEA-86, under the number APAFiS\#4145.

\section{Gut microbiota composition analysis}

Gut microbiota were analyzed in two cohorts (15 WT and 16 CAMLCK ${ }^{T g}$ ) from 8 different WT dams. At sacrifice, colonic contents were stored at $-80^{\circ} \mathrm{C}$. DNA was extracted using the ZR fecal DNA MiniPrep kit (Zymo Research) and adjusted to $1 \mathrm{ng} / \mu \mathrm{L}$. Changes in relative abundance of 24 microbial 16S rRNA gene targets were obtained by qRTPCR using an adapted Gut LowDensity Array platform. ${ }^{5-7}$ A universal bacterial primer set was included as the reference gene. qRTPCR was performed in duplicate on a ViiA7 (Applied Biosystems).

Fluorescence data was imported into LinRegPCR to perform baseline corrections, calculate mean PCR efficiency per amplicon group. and calculate initial quantities. Among the 24 targeted amplicon groups, 6 were not detected in any fecal samples and were removed from the analysis (B. vulgatus, Alistipes spp., Parabacteroidetes distasonis, Roseburia spp., E. coli and A. muciniphila). Normalized $\mathrm{N}_{0}$-values were $\log _{10}$-transformed and processed by MixOmics (v6.1.1) with RStudio (v1.0.44) to build a partial least-squares discriminant analysis (PLS-DA). This multivariate supervised approach projects samples $(X)$ onto a low-dimensional space of latent variables to maximize separation between groups according ( $\mathrm{Y}=$ genotype). Leave-one-out cross-validation was used to select the optimal number of latent variables for PLS-DA models.

\section{Open field test}

Mice explored a 50x50cm arena (illumination 300lux) for 10min. Exploration was automatically assessed using a video tracking system (Bioseb). The percentage of distance traveled and time spent and in the center area $(20 \times 20 \mathrm{~cm})$ and total distance traveled in the entire arena were assessed. 


\section{Colorectal distension (CRD)}

Two $0.08 \mathrm{~mm}$ diameter electrodes were implanted in the abdominal external oblique muscle and a third in the abdominal skin. On postoperative days 3-6, CRD was performed using a balloon catheter (Fogarty $4 \mathrm{~F}$ catheter, $1.1 \mathrm{~cm}$ length, tip $3.5 \mathrm{~cm}$ from the anus) ${ }^{8}$ in $10 \mathrm{sec}$ periods with increasing volumes from $0.02 \mathrm{~mL}$ to $0.10 \mathrm{~mL}$, with $5 \mathrm{~min}$ rest between distensions. Abdominal electromyography activity was registered after the amplification (10000x) and analyzed (Powerlab Chart 5). Basal EMG activity was subtracted from EMG activity registered during distension. Some mice were treated with ML-7 (2 mg/kg i.p.) or naloxone sulfate (2 mg/kg i.p.) $1 \mathrm{~h}$ before CRD. For others, water avoidance stress was induced on a floating platform $(3 \mathrm{~cm} \times 3 \mathrm{~cm})$ in the middle of a water-filled tank $(40 \mathrm{~cm} \times 40 \mathrm{~cm})$ for $1 \mathrm{~h}$ daily over four days. Recovery (30min) preceded CRD.

\section{Gastrointestinal transit}

Animals received $70 \mu \mathrm{L}$ of $100 \mathrm{mg} / \mathrm{ml}$ TRITC-70kDa dextran in tap water by gavage and were sacrificed $1 \mathrm{~h}$ later. ${ }^{9}$ Stomach, small and large intestine were cut in 11 equal parts. Luminal contents of each segment were centrifuged and fluorescence determined. Transit was calculated as the geometric center of the values for each mouse.

\section{Ussing chamber analysis}

Jejunal sections were mounted in Ussing chambers (Physiologic Instruments) filled with Krebs buffer and continuously oxygenated ( $95 \% \mathrm{O} 2,5 \% \mathrm{CO} 2)$. After 1 hour of equilibration, Fluorescein $(1 \mathrm{mg} / \mathrm{mL})$ was added in the apical chamber and fluorescence intensity of the basolateral chamber was measured after 1 hour.

\section{In vivo permeability analysis}

Mice were fasted for 4 hours before gavage with $150 \mu \mathrm{L}$ of $100 \mathrm{mg} / \mathrm{mL}$ FITC-4kDa dextran in tap water. Blood $(200 \mu \mathrm{L})$ was collected after $4 \mathrm{~h}$ and plasma fluorescence determined. 


\section{C-Fos analysis}

Vibratome sections $(40 \mu \mathrm{m})$ were stained using polyclonal rabbit anti-C-Fos (Santa Cruz) and secondary HRP-conjugated goat anti-rabbit antisera (Jackson ImmunoResearch). NDPI images (x20) were obtained (Nanozoomer, Hamamatsu Photonics) and converted into TIFF format using ImageJ (NDPI tools plugin). Regions of interest (ROI) were manually circumscribed using $\mathrm{ROI}$ tools and C-Fos-immunoreactive cells quantified automatically using the particle analysis function (size: 5-20 $\mu \mathrm{m}^{2}$; circularity: 0.5-1). For each animal, 3-6 sections of each brain area were assessed by a blinded observer.

\section{Statistical analysis}

Statistical significance was determined by two-tailed t-test, two-tailed Mann-Whitney U test, or 2-way ANOVA and set at $p<0.05$. For microbial analyses, univariate analysis was realized in parallel to compare each amplicon separately using unpaired t-test followed by the BenjaminiHochberg adjustment of $p$-values for multiple comparisons.

\section{Supplemental references}

1. Su L, et al. Gastroenterology 2009;136:551-63.

2. Weber CR, et al. J Biol Chem 2010;285:12037-46.

3. Edelblum KL, et al. Cell Mol Gastroenterol Hepatol 2017;4:285-297.

4. Yu D, et al. Proc Natl Acad Sci U S A 2010;107:8237-41.

5. Bergstrom A, et al. FEMS Microbiol Lett 2012;337:38-47.

6. Bergstrom A, et al. Appl Environ Microbiol 2014;80:2889-900.

7. Riba A, et al. Gastroenterology 2017;153:1594-1606 e2. 


\title{
Fecal MMP-9: A New Noninvasive Differential Diagnostic and Activity Marker in Ulcerative Colitis
}

\author{
Anita Annaházi, MD, ${ }^{*}+$ Tamás Molnár, MD, PhD, ${ }^{*}$ Klaudia Farkas, MD, PhD, ${ }^{*}$ András Rosztóczy, MD, PhD, * \\ Ferenc Izbéki, MD, PhD, ${ }^{*}$ Krisztina Gecse, MD, PhD, ${ }^{*}$ Orsolya Inczefi, MD, ${ }^{*}$ Ferenc Nagy, MD, PhD,* \\ Imre Földesi, PhD, ${ }^{*}$ Mónika Szücs, MSc, ${ }^{\neq}$Marta Dabek, PhD, ${ }^{+}$Laurent Ferrier, PhD, ${ }^{+}$ \\ Vassilia Theodorou, $\mathrm{PhD}^{+}{ }^{+}$Lionel Bueno, $\mathrm{PhD}{ }^{+}$Tibor Wittmann, MD, PhD, ${ }^{*}$ and Richárd Róka, MD, PhD*
}

Background: Ulcerative colitis (UC) is characterized by frequent relapses, with the presence of colorectal inflammation and mucosal lesions. Matrixmetalloprotease 9 (MMP-9) is elevated in colonic biopsies, urine, and blood plasma of UC patients. MMP-9 has been suggested as a predictor of UC in the urine of children; however, $20 \%$ of the controls tested positive. So far, fecal MMP-9 levels have never been measured. Our aims were: 1) to compare fecal MMP-9 levels in UC patients to control subjects and a functional gastrointestinal disorder characterized by diarrhea (IBS-D); 2) to test the correlation between UC disease activity and fecal levels of MMP-9; and 3) to correlate fecal MMP-9 levels with a known fecal marker of UC activity, calprotectin.

Methods: UC $(n=47)$, IBS-D $(n=23)$ patients, and control subjects $(n=24)$ provided fecal samples for MMP-9 analysis. In UC patients, disease severity was evaluated by the Mayo score. Fecal MMP-9 and calprotectin levels were measured by enzyme-linked immunosorbent assay and lateral flow assay, respectively.

Results: MMP-9 was undetectable or $\leq 0.22 \mathrm{ng} / \mathrm{mL}$ in the feces of all controls and IBS-D patients. In UC patients, fecal MMP-9 levels significantly correlated with the overall Mayo score $(P<0.001)$, the endoscopic score $(P<0.001)$, and the serum C-reactive protein levels $(P=0.002)$. Additionally, in UC patients fecal MMP-9 levels showed a significant correlation with a known disease activity marker, fecal calprotectin $(P=0.014)$.

Conclusions: These results highlight fecal MMP-9 as a useful tool in the differential diagnosis of diarrheic disorders and in the noninvasive evaluation of disease activity and mucosal healing in UC.

(Inflamm Bowel Dis 2013;19:316-320)

Key Words: ulcerative colitis, MMP-9, disease activity score, calprotectin

U lcerative colitis (UC) is a chronic, relapsing inflammatory bowel disease (IBD) characterized by continuous colonic mucosal ulceration and diarrhea during relapses. The differentiation of UC from functional disorders such as diarrhea predominant irritable bowel syndrome (IBS-D) is clinically challenging, as symptoms may include altered bowel habits and abdominal pain in both diseases and activity signs can mix with anxiety-driven symptoms as well in UC. Several

Received for publication March 20, 2012; Accepted March 29, 2012

From the *First Department of Medicine, University of Szeged, Szeged, Hungary; ${ }^{\dagger}$ Toxalim UMR 1331 INRA/INP/UPS, Neuro-Gastroenterology \& Nutrition Unit, Toulouse, France; and Department of Medical Physics and Informatics, University of Szeged, Szeged, Hungary.

The first two authors contributed equally to this work.

Anita Annaházi was a recipient of a postdoctoral fellowship from INRA. Supported by an institutional grant from INRA and TÁMOP (TÁMOP-4.2.1/B-09/1/ KONV-2010-0005)

Reprints: Anita Annaházi, MD, First Department of Medicine, University of Szeged, Szeged. Koranyi fasor 8-10., 6720, Hungary (e-mail: annanita3@ yahoocom).

Copyright (C) 2013 Crohn's \& Colitis Foundation of America, Inc.

DOI 10.1002/ibd.22996

Published online 1 May 2012. attempts have been made to establish noninvasive markers that can clearly distinguish IBD from functional disorders and reveal strong correlation not only with clinical activity, but also with the endoscopic picture of the mucosa in UC. Among biochemical laboratory markers serum C-reactive protein (CRP) is the most studied ${ }^{1}$; however, while the activity of Crohn's disease (CD) is accompanied by a strong CRP response in most patients, relapses in UC are characterized by only a modest or absent CRP increase, ${ }^{2}$ depending mostly on the extension of the involved area; the sensitivity of CRP in the detection of UC is only $50 \%-70 \% .^{3-5}$

The pathogenesis of UC includes the complex dysregulation of mucosal immune cells ${ }^{6}$ accompanied by the invasion of neutrophils, which leads to the formation of crypt abscesses and to the dysfunction of the colonic epithelial barrier. ${ }^{7}$ During transmigration, neutrophils reach the colonic lumen and can be detected in the stool, ${ }^{8}$ similar to their secreted mediators, such as polymorphonuclear elastase, calprotectin, or Cat-G. ${ }^{9,10}$ Therefore, fecal biomarkers that reflect neutrophil activation seem promising in UC, and fecal calprotectin has been identified as a marker to discriminate IBD patients from irritable bowel syndrome (IBS) 
patients or controls, ${ }^{10,11}$ and its fecal level correlates with disease activity. $^{12,13}$

Matrix metalloproteases (MMPs) 1, 2, 3, and 9 are also released from neutrophils in IBD, and they are significantly elevated in colonic biopsies from UC patients compared to controls, with a higher increase in the ulcerated than in nonulcerated regions. ${ }^{14}$ Among these MMPs, MMP-9 was the most abundant in UC biopsies. ${ }^{14}$ Additionally, elevated MMP-2 and MMP-9 levels were detected in the urine of pediatric patients suffering from either UC or CD, and these MMPs were suggested as noninvasive biomarkers in the diagnosis of IBD. ${ }^{15}$ Nevertheless, no study has so far focused on fecal MMP levels in UC compared with IBS-D. Furthermore, the correlation of MMPs with disease severity, clinical, and endoscopic activity has never been evaluated.

Therefore, in our study we aimed to compare fecal MMP-9 levels in UC patients with those of IBS-D patients and controls. As a second step, we correlated fecal MMP-9 levels of UC patients with disease severity based on clinical and colonoscopic scores. In the last part of our study, we compared the diagnostic value of MMP-9 and fecal calprotectin levels in a separate group of UC patients.

\section{MATERIAL AND METHODS}

\section{Patient Selection and Sample Collection}

Overall, 94 patients were enrolled in the study. Healthy controls ( $n=24$; male/female: $9 / 15$; mean age: 49.3 years), UC ( $n=47$; male/female: 19/28; mean age: 48.0 years), and IBS-D ( $n=23$; male/female: $4 / 19$; mean age: 49.4 years) patients were recruited to provide fecal samples at the First Department of Medicine, Szeged, Hungary.

IBS-D patients fulfilled the Rome III criteria. Organic gastrointestinal (GI) disorders were excluded by detailed blood analyses and stool cultures, serologic assays for celiac disease, lactose-hydrogen breath test, and colonoscopy.

UC patients were evaluated clinically and endoscopically. The activity of the disease was determined by clinical (partial and total Mayo score) and endoscopic (Mayo endoscopic subscore) scores. Based on the total Mayo score, 12 patients were determined as inactive, 17 as mildly active, 9 as moderately active, and 9 as severely active. At the time of the endoscopy, blood samples were taken from all patients for the determination of platelet (PLT) and leukocyte count (WBC), CRP, erythrocyte sedimentation rate (ESR), serum iron level $(\mathrm{Fe})$, and hemoglobin level $(\mathrm{Hgb})$. Fecal samples were collected from all patients 2-4 days before the colonoscopy and were frozen to $-20^{\circ} \mathrm{C}$ within 1 hour after defecation.

\section{Measurement of Fecal MMP-9}

Samples were thawed at $4^{\circ} \mathrm{C}$. One $\mathrm{g}$ of each fecal sample was diluted, mixed, and homogenized in $4 \mathrm{~mL}$ of ice-cold Tris-buffer $(0.15 \mathrm{M} \mathrm{NaCl}+20 \mathrm{mM}$ Tris-HCl, $\mathrm{pH}$ 8.3). After centrifugation $\left(10 \mathrm{~min}, 4500 \mathrm{rpm}, 4^{\circ} \mathrm{C}\right)$, pellets were discarded and supernatants were recentrifuged $\left(10 \mathrm{~min}, 10,000 \mathrm{~g} ; 4^{\circ} \mathrm{C}\right)$. The final supernatants were filtered by $0.8 \mu \mathrm{m}$ pore-sized syringe filters and the aliquots were stored at $-20^{\circ} \mathrm{C}$ until analyzed. MMP-9 was measured by a quantitative enzyme-linked immunosorbent assay (Quantikine, R\&D Systems, Abingdon, UK).

\section{Measurement of Fecal Calprotectin}

In the second part of this study, fecal calprotectin was measured in a subset of UC patients $(n=24$, male/ female: $10 / 14$, mean age: 45.7 years). For calprotectin measurements, fecal specimens were thawed at $4{ }^{\circ} \mathrm{C}$ and calprotectin level was determined by a quantitative lateral flow assay (Quantum Blue, Bühlmann Laboratories, Schönenbuch, Switzerland) according to the manufacturer's instructions.

\section{Statistical Analysis}

The results are presented as mean \pm standard deviation. Due to the nonnormal distribution of the data, a Kruskal-Wallis test was used to compare fecal MMP-9 levels of the three groups (control, IBS-D, and UC) and four different groups of UC related to disease activity. Correlation between MMP-9 levels and disease activity scores or blood test results were determined by Spearman correlation. Correlation between MMP-9 and calprotectin were also tested by Spearman correlation. The cutoff levels, specificity, and sensitivity were calculated using receiver operating characteristic (ROC) analysis. In UC patients, active disease has been determined as total Mayo score $>3$ and Mayo endoscopic subscore $>0$. The cutoff level was calculated in case the area under the ROC curve (AUC) value was above 0.9. The cutoff level was determined by the maximum value of Youden's index (sensitivity + specificity-1).

\section{Ethical Considerations}

The study protocol was approved by the Ethical Committee of the University of Szeged. All subjects provided written and informed consent to participate.

\section{RESULTS}

\section{Fecal MMP-9 Levels Between Groups}

There were no significant differences between the three groups (control, IBS-D, and UC) regarding age $(P=0.916)$. Fecal MMP-9 contents were significantly different between the three groups (Fig. 1, $P<0.001$ ). Indeed, specimens originating from healthy subjects had very low MMP-9 levels; nearly half of them were found to be below the detection limit of the assay (mean: $0.0208 \pm 0.045 \mathrm{ng} / \mathrm{mL}$ ). Similarly, IBS-D patients had low fecal MMP-9 levels (IBS-D: $0.0017 \pm 0.008 \mathrm{ng} / \mathrm{mL}$ ). However, in the UC group the average MMP-9 level was significantly higher (UC: $15.65 \pm 20.14 \mathrm{ng} / \mathrm{mL}$ ). To distinguish UC patients from healthy controls or IBS-D patients, the cutoff level was determined at $0.245 \mathrm{ng} / \mathrm{mL}(\mathrm{AUC}=0.939)$. By means of this cutoff, sensitivity was $85.1 \%$ and specificity $99.99 \%$ (Fig. 1). 


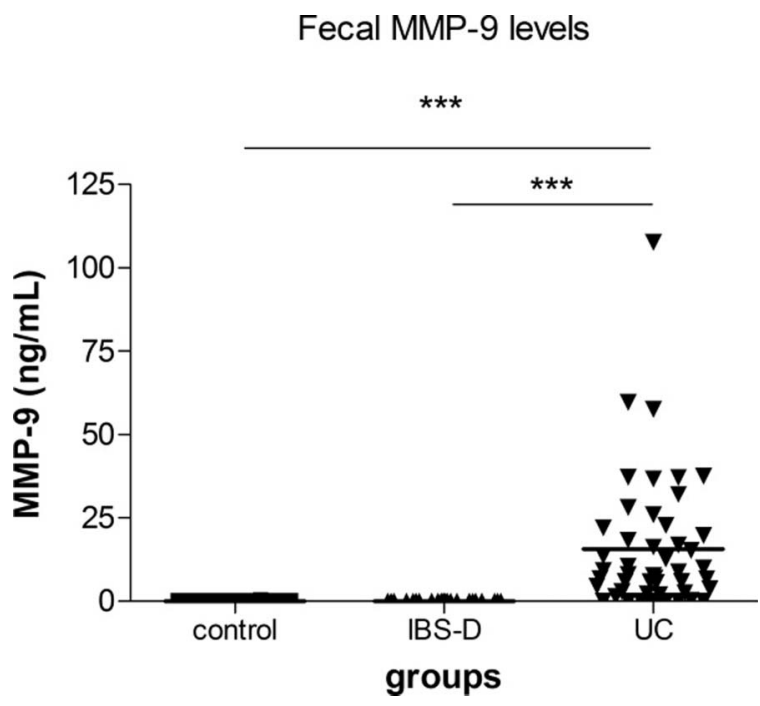

FIGURE 1. Fecal MMP-9 levels in the three different groups: control, IBS-D, UC. Fecal MMP-9 concentration is significantly elevated in UC patients compared to controls or IBS-D patients ( $\left.{ }^{* * *} P<0.001\right)$.

\section{Correlation of Fecal MMP-9 Levels with Disease Activity in UC}

MMP-9 activity significantly correlated with the overall Mayo score $(\mathrm{R}=0.616 ; P<0.001$; AUC $=0.905)$. Fecal MMP9 levels were significantly lower in case of inactive disease compared to moderately or highly active (Fig. 2). Further, within the components of the Mayo score a strong correlation was observed with the endoscopic evaluation of the colorectal mucosa $(R=$ 0.653; $P<0.001$, AUC $=0.900$ ). To separate active UC patients from those in remission, the cutoff level of MMP-9 was determined at $2.38 \mathrm{ng} / \mathrm{mL}$. This cutoff resulted in a sensitivity of $97.1 \%$ and a specificity of $83.3 \%$. Correlation of fecal MMP-9 with laboratory findings is illustrated in Table 1.

\section{Correlation of Fecal MMP-9 Concentration with Fecal Calprotectin Levels in UC}

In a subset of 24 UC patients, fecal calprotectin levels were also determined. Significant correlation was found between the fecal MMP-9 and calprotectin levels $(R=0.495 ; P=0.014)$. In this subset of patients the correlation of endoscopic and overall Mayo scores was calculated with both MMP-9 and calprotectin. In this smaller cohort of patients, fecal MMP-9 levels showed a slightly stronger correlation with the endoscopic component of the Mayo score than calprotectin $(R=0.587 ; P=0.003, \mathrm{AUC}=$ 0.895 for calprotectin and $R=0.653 ; P=0.002$, AUC $=0.939$ for MMP-9), and a higher correlation with the overall Mayo score than calprotectin $(R=0.587 ; P=0.016, \mathrm{AUC}=0.895$ for calprotectin and $R=0.718 ; P=0.002$, AUC $=0.905$ for MMP-9).

\section{DISCUSSION}

To the best of our knowledge this study is the first to report elevated fecal MMP-9 levels in patients with active UC compared to functional (IBS-D) patients or controls. In UC patients fecal MMP-9 levels are excellent predictors of disease activity and show a significant correlation with the clinical and endoscopic scores. Fecal MMP-9 levels correlate significantly with fecal calprotectin, a well-known marker of disease activity in UC. MMPs are a family of $\mathrm{Zn}^{2+}$-containing endopeptidases capable of degradation of the extracellular matrix. They are secreted by various cell types, such as fibroblasts, mesenchymal cells, tumor cells, and several inflammatory cells like monocytes, lymphocytes, and neutrophils. MMP-9 is a gelatinase that represents $\approx 30 \%$ of protein content of neutrophil granules. ${ }^{16}$ In a murine experimental model, it has been shown that epithelial cells and granulocytes are the main sources of MMP-9 in colitic tissue, and exogenous MMP-9 impairs epithelial wound healing and cell attachment in vitro. ${ }^{17}$

Urinal MMP-2 and MMP-9 levels have been suggested to discriminate children with IBD from controls; nevertheless, urine samples were found positive for MMP-2 in 25\%, for MMP-9 in $20 \%$ of control individuals, leading to false positivity in the diagnosis. ${ }^{15}$ Further, as MMPs play important roles in cancer development and metastasis formation, ${ }^{18}$ urinary MMPs were found elevated in various cancer types including prostate, bladder, breast, brain, and hematologic malignancies. ${ }^{19-22}$ These findings point out that urinary MMPs are not specific for IBD. We hypothesize that fecal MMP level may be more characteristic of GI pathologies than of other organ-specific or systemic disorders, although its specificity to UC compared to other colon diseases needs to be verified. In colonic biopsies, MMP-9 levels were significantly increased in UC patients, and less significantly

\section{Fecal MMP-9 in UC patients}

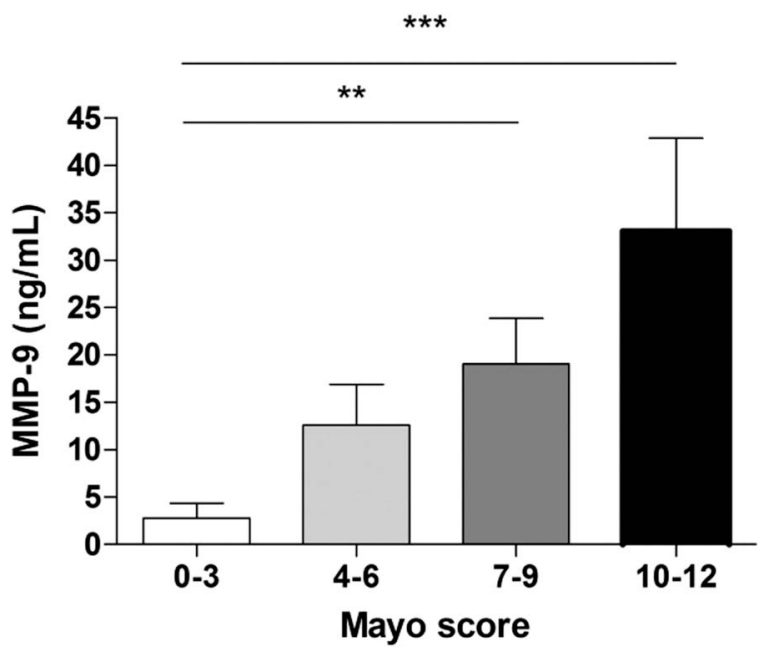

FIGURE 2. Fecal MMP-9 levels in UC patients depending on disease activity. Fecal MMP-9 concentration is significantly elevated in UC patients with moderate $\left({ }^{* *} P<0.01\right)$ and severe $\left.{ }^{* * * P} P<0.001\right)$ activity compared to those in remission. 
TABLE 1. Correlation of Fecal MMP-9 Levels with Blood Test Results

\begin{tabular}{ccccccc}
\hline & \multicolumn{5}{c}{ Blood Test Results } \\
\cline { 2 - 6 } & CRP & ERS & Fe & WBC & Hgb & PLT \\
\hline MMP-9 & $\mathrm{R}=0.456$ & $\mathrm{R}=0.228$ & $\mathrm{R}=0.454$ & $\mathrm{R}=0.404$ & $\mathrm{R}=0.151$ \\
& $P=0.002$ & $P=0.137$ & $P=0.003$ & $P=0.005$ & $P=0.317$ & $\mathrm{R}=0.296$ \\
& & & & $P=0.046$
\end{tabular}

Significance is accepted at $P<0.05$.

increased in CD patients compared to controls. ${ }^{14}$ Based on colonic biopsies, a recent study demonstrated that in contrast to UC, MMP-9 is not involved in the inflammatory process of lymphocytic colitis and collagenous colitis. ${ }^{23}$ By means of a cutoff level of fecal MMP-9 at $0.245 \mathrm{ng} / \mathrm{mL}$, none of our controls and IBS-D patients tested positive. Nevertheless, fecal MMP-9 negativity does not rule out IBD, as seven UC patients, all in remission, were also below the cutoff level. It is important to note that none of them had colonic ulcerations on endoscopy (Mayo endoscopic subscore $\leq 1$ ). Therefore, our data suggest that low fecal MMP-9 level is a negative predictor of mucosal lesions. Fecal MMP-9 positivity suggests the need of further evaluation of inflammatory diseases or other GI pathologies.

Interestingly, in an earlier study on CD patients, MMP-9 plasma levels were found elevated compared to controls, but no correlation could be observed with the Clinical Disease Activity Index (CDAI). ${ }^{24}$ Similarly, it has been demonstrated that other disease activity markers, such as calprotectin, ${ }^{25}$ lactoferrin, or CRP, and even endoscopic appearance correlate poorly with CDAI. $^{26}$ In our study, however, fecal MMP-9 concentration showed a significant correlation with the clinical and endoscopic scores in UC patients. Additionally, fecal MMP-9 level significantly correlated with CRP, a laboratory value referring to inflammation, widely used as a disease activity marker in the blood. ${ }^{1}$

In the last part of our study we compared fecal MMP-9 to an established fecal marker in UC, calprotectin, and a significant correlation was found between them. In some centers, fecal calprotectin is already used as a routine test to discriminate IBS from IBD. ${ }^{27}$ These data suggest that fecal MMP-9 can be a novel marker to help in the differential diagnosis of patients with diarrhea and abdominal pain. Further, it could be a new complementary noninvasive method to assess mucosal inflammation and lesions in UC patients.

\section{ACKNOWLEDGMENT}

The authors thank Valérie Bezirard and Mathilde Leveque for help in the measurements.

\section{REFERENCES}

1. Vermeire S, Van Assche G, Rutgeerts P. Laboratory markers in IBD: useful, magic, or unnecessary toys? Gut. 2006;55:426-431.
2. Saverymuttu SH, Hodgson HJ, Chadwick VS, et al. Differing acute phase responses in Crohn's disease and ulcerative colitis. Gut. 1986;27: 809-813.

3. Poullis AP, Zar S, Sundaram KK, et al. A new, highly sensitive assay for C-reactive protein can aid the differentiation of inflammatory bowel disorders from constipation- and diarrhoea-predominant functional bowel disorders. Eur J Gastroenterol Hepatol. 2002;14: 409-412.

4. Shine B, Berghouse L, Jones JE, et al. C-reactive protein as an aid in the differentiation of functional and inflammatory bowel disorders. Clin Chim Acta. 1985;148:105-109.

5. Schoepfer AM, Trummler M, Seeholzer P, et al. Discriminating IBD from IBS: comparison of the test performance of fecal markers, blood leukocytes, CRP, and IBD antibodies. Inflamm Bowel Dis. 2008;14: 32-39.

6. Kucharzik T, Maaser C, Lugering A, et al. Recent understanding of IBD pathogenesis: implications for future therapies. Inflamm Bowel Dis. 2006; 12:1068-1083.

7. Kucharzik T, Walsh SV, Chen J, et al. Neutrophil transmigration in inflammatory bowel disease is associated with differential expression of epithelial intercellular junction proteins. Am J Pathol. 2001;159: 2001-2009.

8. Saverymuttu SH, Chadwick VS, Hodgson HJ. Granulocyte migration in ulcerative colitis. Eur J Clin Invest. 1985;15:60-63.

9. Dabek M, Ferrier L, Roka R, et al. Luminal cathepsin g and proteaseactivated receptor 4: a duet involved in alterations of the colonic epithelial barrier in ulcerative colitis. Am J Pathol. 2009; 175:207-214.

10. Roseth AG, Fagerhol MK, Aadland E, et al. Assessment of the neutrophil dominating protein calprotectin in feces. A methodologic study. Scand $J$ Gastroenterol. 1992;27:793-798.

11. Roseth AG, Aadland E, Jahnsen J, et al. Assessment of disease activity in ulcerative colitis by faecal calprotectin, a novel granulocyte marker protein. Digestion. 1997;58:176-180.

12. Costa F, Mumolo MG, Bellini M, et al. Role of faecal calprotectin as non-invasive marker of intestinal inflammation. Dig Liver Dis. 2003;35: 642-647.

13. Tibble J, Teahon K, Thjodleifsson B, et al. A simple method for assessing intestinal inflammation in Crohn's disease. Gut. 2000;47:506-513.

14. Baugh MD, Perry MJ, Hollander AP, et al. Matrix metalloproteinase levels are elevated in inflammatory bowel disease. Gastroenterology. 1999;117:814-822.

15. Manfredi MA, Zurakowski D, Rufo PA, et al. Increased incidence of urinary matrix metalloproteinases as predictors of disease in pediatric patients with inflammatory bowel disease. Inflamm Bowel Dis. 2008; 14:1091-1096.

16. Opdenakker G, Van den Steen PE, Van Damme J, Gelatinase B: a tuner and amplifier of immune functions. Trends Immunol. 2001;22:571579 .

17. Castaneda FE, Walia B, Vijay-Kumar M, et al. Targeted deletion of metalloproteinase 9 attenuates experimental colitis in mice: central role of epithelial-derived MMP. Gastroenterology. 2005;129:1991-2008.

18. Nakajima M, Welch DR, Wynn DM, et al. Serum and plasma M(r) 92,000 progelatinase levels correlate with spontaneous metastasis of rat 13762NF mammary adenocarcinoma. Cancer Res. 1993;53:5802-5807.

19. Chan LW, Moses MA, Goley E, et al. Urinary VEGF and MMP levels as predictive markers of 1-year progression-free survival in cancer patients 
treated with radiation therapy: a longitudinal study of protein kinetics throughout tumor progression and therapy. J Clin Oncol. 2004;22: 499-506.

20. Fernandez CA, Yan L, Louis G, et al. The matrix metalloproteinase-9/ neutrophil gelatinase-associated lipocalin complex plays a role in breast tumor growth and is present in the urine of breast cancer patients. Clin Cancer Res. 2005;11:5390-5395

21. Moses MA, Wiederschain D, Loughlin KR, et al. Increased incidence of matrix metalloproteinases in urine of cancer patients. Cancer Res. 1998; 58:1395-1399.

22. Smith ER, Zurakowski D, Saad A, et al. Urinary biomarkers predict brain tumor presence and response to therapy. Clin Cancer Res. 2008; $14: 2378-2386$.
23. Lakatos G, Sipos F, Miheller P, et al. The behavior of matrix metalloproteinase-9 in lymphocytic colitis, collagenous colitis and ulcerative colitis. Pathol Oncol Res. 2012;18:85-91.

24. Kossakowska AE, Medlicott SA, Edwards DR, et al. Elevated plasma gelatinase A (MMP-2) activity is associated with quiescent Crohn's Disease. Ann N Y Acad Sci. 1999;30;878:578-580.

25. Gaya DR, Lyon TD, Duncan A, et al. Faecal calprotectin in the assessment of Crohn's disease activity. QJM. 2005;98:435-441.

26. Jones J, Loftus EV Jr, Panaccione R, et al. Relationships between disease activity and serum and fecal biomarkers in patients with Crohn's disease. Clin Gastroenterol Hepatol. 2008;6:1218-1224.

27. Poullis A, Foster R, Mendall MA, et al. Emerging role of calprotectin in gastroenterology. J Gastroenterol Hepatol. 2003;18:756-762. 


\title{
A pilot study on faecal MMP-9: a new noninvasive diagnostic marker of colorectal cancer
}

\author{
Anita Annaházi ${ }^{*}, 1,5$, Szabolcs Ábrahám ${ }^{2,5}$, Klaudia Farkas ${ }^{1}$, András Rosztóczy ${ }^{1}$, Orsolya Inczefi ${ }^{1}$, Imre Földesi ${ }^{1}$, \\ Mónika Szücs ${ }^{3}$, Mariann Rutka ${ }^{1}$, Vassilia Theodorou ${ }^{4}$, Helene Eutamene ${ }^{4}$, Lionel Bueno ${ }^{4}$, György Lázár ${ }^{2}$, Tibor \\ Wittmann ${ }^{1}$, Tamás Molnár ${ }^{1}$ and Richárd Róka ${ }^{1}$ \\ ${ }^{1}$ First Department of Medicine, University of Szeged, Korányi Fasor 8-10, Szeged 6720, Hungary; ${ }^{2}$ Department of Surgery, \\ University of Szeged, Pf. 427, Szeged 6701, Hungary; ${ }^{3}$ Department of Medical Physics and Informatics, University of Szeged, \\ Korányi Fasor 9, Szeged 6720, Hungary and ${ }^{4}$ Toxalim UMR 1331 INRA/INP/UPS, Neuro-Gastroenterology and Nutrition Unit, 180, \\ Chemin de Tournefeuille, BP.93173, Toulouse Cedex 3, 31027, France
}

Background: Colorectal cancer (CRC) is one of the leading malignancies worldwide, therefore cheap noninvasive screening methods are of great importance. Matrix-metalloproteinase-9 (MMP-9) has a role in the progression of CRC, and its level is elevated in tumour biopsies. Faecal MMP-9 levels are increased in active ulcerative colitis patients, but in CRC patients, they have never been measured. We aimed to assess the faecal MMP-9 levels in patients undergoing total colonoscopy according to endoscopic and histological diagnosis.

Methods: One hundred and nine patients provided faecal samples for MMP-9 analysis. A total colonoscopy was performed; suspicious lesions were evaluated by histology. Faecal MMP-9 levels were measured by ELISA.

Results: The number of patients allocated to different groups were: negative/diverticulosis: 34 (referred to as controls); hyperplastic polyps: 15; adenomas: 32 (22 at high risk); and CRC: 28. Faecal MMP-9 was significantly increased in CRC compared with all other groups $(P<0.001)$. Faecal MMP-9 was suitable to distinguish CRC patients from controls (sensitivity: 89.3\%; specificity: 91.2\%). By means of a lower cutoff level, faecal MMP-9 identified high-risk adenomas besides CRC (sensitivity: 76\%; specificity: $85.3 \%)$. This lower cutoff level screened $59 \%$ of high-risk adenomas.

Conclusions: Faecal MMP-9 may be a promising new noninvasive marker in CRC.

Colorectal cancer (CRC) represents a major cause of morbidity and mortality in Western societies, and the incidence in developing countries is also rapidly growing (Sung et al, 2008; Siegel et al, 2012). In Europe, CRC is the second most frequent type of malignancy among males and females, with an estimated incidence of $>400000$ new cases per year (Ferlay et al, 2013). The high frequency of the disease and the fact that the prognosis correlates well with the stage at diagnosis makes CRC screening extremely important. Colonoscopy is the gold standard for CRC screening by its high sensitivity and specificity but has also disadvantages, such as risk of complications, high costs and lower acceptance by patients as a screening method. Therefore, noninvasive methods are required to identify patients at high risk of CRC where colonoscopy needs to be performed.

CRC screening recommendations vary between countries but mostly consist of an annual or biannual faecal occult blood test (FOBT) combined with colonoscopy in case of positivity or a colonoscopy every 10 years, in the population aged $>50$ years

\footnotetext{
*Correspondence: Dr A Annaházi; E-mail: annanita3@yahoo.com

${ }^{5}$ These authors contributed equally to this work.
}

Received 23 March 2015; revised 6 January 2016; accepted 20 January 2016; published online 23 February 2016

(c) 2016 Cancer Research UK. All rights reserved 0007 - 0920/16 
(Binefa et al, 2014). Two basic types of FOBT exist, based on the imperceptible intermittent leakage of blood from CRC and highrisk adenomas in stool (Binefa et al, 2014). The guaiac test (gFOBT) detects the peroxidase activity in the haemoglobin haeme subunit, therefore it is nonspecific for human haemoglobin and theoretically requires dietary restrictions a few days before performing the test, but large studies have found no significant advantage of the dietary modifications, therefore lately they are not recommended by many authors (Konrad, 2010). A minimum of two faecal samples are necessary from different stools to obtain an appropriate sensitivity. Sensitivity for CRC ranges from $6.2 \%$ to $83.3 \%$, depending on the test used, while specificity is $>80 \%$ (Binefa et al, 2014). The immunochemical faecal occult blood test (FIT) is based on specific monoclonal or polyclonal antibodies against human haemoglobin, thus it does not require dietary alterations. Contrary to gFOTB, the analysis by FIT can be automated and its threshold value is adjustable by the user. According to the literature, the sensitivity of FIT is extremely variable, from $5.4 \%$ to nearly $98 \%$, depending on the test used (Binefa et al, 2014). Specificity can range from $77 \%$ to $99 \%$. The European Guidelines for quality assurance of CRC recommends the use of FIT rather than gFOBT (Halloran et al, 2012). However, reliable tests with a less variable sensitivity and specificity are still lacking.

Matrix-metalloproteinases (MMPs) are a family of $\mathrm{Zn}^{2+}$. containing endopeptidases, secreted by various cell types, such as tumour cells, mesenchymal cells, fibroblasts and inflammatory cells, such as monocytes, lymphocytes and neutrophils. Increased expression of MMP-9 has been detected in tissue samples from rectal carcinoma and colon cancer, where it correlates with poor prognosis (Unsal et al, 2008; Araujo et al, 2015; Yang et al, 2014). Recently, we have shown that faecal MMP-9 levels are elevated in active ulcerative colitis (UC) patients compared with patients with a functional gastrointestinal disorder (diarrhoea-predominant irritable bowel syndrome) or healthy controls (Annahazi et al, 2013). Faecal MMP-9 levels were found to be a predictor of disease activity in UC patients (Annahazi et al, 2013), and had a high specificity in the diagnosis of pouchitis (Farkas et al, 2014). However, faecal MMP-9 levels in CRC patients have not been investigated.

Based on this background, we aimed to measure faecal MMP-9 levels in patients undergoing colonoscopic examination in our hospital and to analyse the results according to the different colonoscopic and histological findings.

\section{MATERIALS AND METHODS}

Ethical considerations. The study protocol was approved by the Ethical Committee of the University of Szeged, Szeged, Hungary. All subjects provided written and informed consent to participate.

Patient selection and sample collection. Overall, 109 patients (male/female: 58/51, age: 29-91 years, mean: 66.0 years) referred to our specialist colorectal unit in the First Department of Medicine, Szeged, Hungary were enrolled in the study, who all underwent colonoscopy. No patients had a history of UC or a history of other cancers. One patient, who was later identified as a rectal adenocarcinoma patient, had liver metastases. Faecal samples were collected from all patients 2-4 days before the colonoscopy and were frozen to $-20^{\circ} \mathrm{C}$ within $1 \mathrm{~h}$ after defaecation. All colonoscopies were completed until reaching the terminal ileum. During colonoscopy, biopsies were taken from all suspect lesions, and histology was performed. Based on the colonoscopic and histological results, patients were allocated to five groups: negative, diverticulosis, hyperplastic polyp, adenoma, and CRC. Adenomas were considered at increased risk of malignisation when one of the followings was fulfilled: polyp size $>10 \mathrm{~mm}$; villous adenoma or tubulovillous adenoma with at least $20 \%$ villous component; highgrade dysplasia; or multiple adenoma $(>3)$.

Validation assay. Our group was the first to use the commercial Human Quantitative Enzyme-Linked Immunosorbent Assay Kit from R\&D Systems (Abingdon, UK, Cat. No. DMP900) for the measurement of MMP-9 levels in faeces. Therefore, we have performed a validation assay to test whether the faecal matrix itself has an influence on MMP-9 determination and whether the kit from R\&D Systems is able to determine MMP-9 from human faecal extracts.

Active human MMP-9 full-length protein (Abcam LTD, Cambridge, UK; Cat. No.: ab157344; LOT No: GR218764-1) was added to the extraction medium. Control faecal samples were weighed and $1 \mathrm{ml}$ extraction medium with or without added MMP-9 was given to $0.25 \mathrm{mg}$ sample (the sample to extraction medium ratio was $1: 4$ ). The samples were immediately homogenised for $2 \times 30 \mathrm{~s}$ and incubated for $10 \mathrm{~min}$ at room temperature. Subsequently, all homogenates were centrifuged at $1500 \mathrm{~g}$ for $10 \mathrm{~min}$ followed by a second centrifugation of the supernatants from the first centrifugation step at $10000 \mathrm{~g}$ for $10 \mathrm{~min}$. The final supernatants were processed for linearity (parallelism) test. The extract containing added MMP-9 was serially diluted $1+1$ to get $50,25,12.5$ and $6.25 \%$ of the original MMP-9 content with the extract of the same faeces containing no added MMP-9. Then all samples, including $100 \%$ of added MMP-9 extract and the native control extract without added MMP-9, were subjected to MMP-9 measurement by the above-mentioned ELISA kit for human MMP-9. The kit is able to detect the human $92 \mathrm{kDa}$ Pro-MMP-9 and $82 \mathrm{kDa}$ active form of MMP-9 as stated by the manufacturer. The added MMP-9 protein obtained for this evaluation was the $92 \mathrm{kDa}$ Pro-MMP-9.

Measurement of faecal MMP-9. Samples were stored on $-20^{\circ} \mathrm{C}$ for a maximum of 3 months and then thawed at $4{ }^{\circ} \mathrm{C}$ for 1 day. In all, $1.0 \mathrm{~g}$ of each faecal sample was diluted, mixed and homogenised in $4 \mathrm{ml}$ of ice-cold Tris-buffer $(0.15 \mathrm{M} \mathrm{NaCl}+20 \mathrm{~mm}$ Tris- $\mathrm{HCl}$, $\mathrm{pH}: 8.3)$. After centrifugation ( $10 \mathrm{~min}, 4500$ r.p.m., $4{ }^{\circ} \mathrm{C}$ ), pellets were discarded and supernatants were recentrifuged $(10 \mathrm{~min}$, $\left.10000 \mathrm{~g} ; 4^{\circ} \mathrm{C}\right)$. The final supernatants were filtered by $0.8-\mu \mathrm{m}$ pore-sized syringe filters, and the aliquots were stored at $-20^{\circ} \mathrm{C}$ until analysed. MMP-9 was measured by the quantitative enzymelinked immunosorbent assay described above.

Statistical analysis. Continuous data were presented as mean \pm s.d. Kruskal-Wallis test was used to compare faecal MMP-9 levels of the different groups (control, hyperplastic polyp, adenoma and CRC). MMP-9 levels of high- and low-risk adenoma subgroups were analysed by the Mann-Whitney test. The different subgroups according to tumour localisation or stage were compared with the Kruskal-Wallis test. Differences were considered statistically significant when $P<0.05$.

Receiver operating characteristic (ROC) curve analysis was performed to determine the predictive power of the MMP-9 level. Area under ROC curve (AUC), confidence interval for AUC, sensitivity and specificity were calculated. The optimal cutoff values were determined using Youden's index, if the AUC was $>0.8$ (Baker and Kramer, 2007) The cutoff level was determined by the maximum value of Youden's index (sensitivity + specificity -1$)$. Statistical analyses were performed by the $\mathrm{R}$ (version 3.1.2) statistical software (https://www.r-project.org/).

\section{RESULTS}

Validation assay. The concentrations measured in $\mathrm{ng} \mathrm{ml}^{-1}$ are shown in Figure 1. The figure clearly shows a linear concentration 
change corresponding to dilution. It indicates that (i) the faecal matrix itself has no influence on MMP-9 determination; and (ii) the kit from R\&D Systems is able to determine MMP-9 from human faecal extracts.

Faecal MMP-9 levels between groups. Seventeen patients (male/ female: 8/9; mean age: 59.7 years) were found negative on total colonoscopy and 17 patients had no other finding from noninflamed diverticulosis (male/female: 3/14; mean age: 71.8 years). There was no significant difference between these two groups in terms of faecal MMP-9 levels $(P=0.49)$, therefore these 34 patients were used as polyp-free/non-malignant controls (mean age: 65.7 years). Out of the other patients, 15 had hyperplastic polyps (male/female: 9/6; mean age: 62.3 years), 32 had adenomas (male/female: 21/11; mean age: 67.0) and 28 had CRC (all adenocarcinomas; male/female: 17/11; mean age: 67.2 years) according to the endoscopic and histological examinations. There were no statistically significant differences between the four groups (controls, hyperplastic polyps, adenomas, CRC) regarding the age. Faecal MMP-9 levels were significantly different between the four groups (Figure 2, $P<0.0001$ ). Faecal samples from control patients had very low MMP-9 levels (mean: $0.118 \pm 0.057 \mathrm{ng} \mathrm{ml}^{-1}$ ). Patients with hyperplastic polyps also presented with low faecal MMP-9 levels (mean: $0.112 \pm 0.073 \mathrm{ng} \mathrm{ml}^{-1}$ ). Adenoma patients

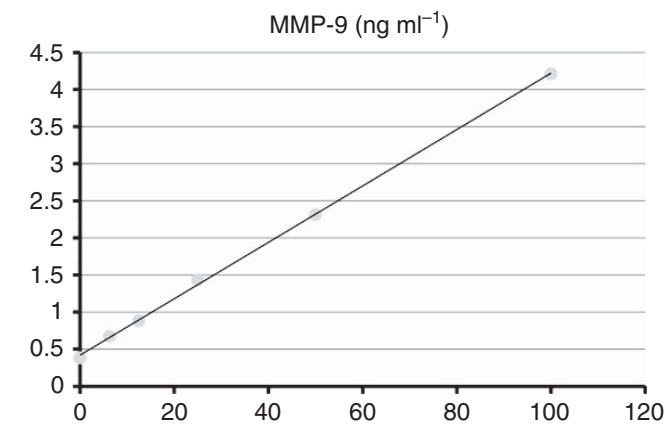

Figure 1. MMP-9 concentration levels at different dilutions in the validation assay.

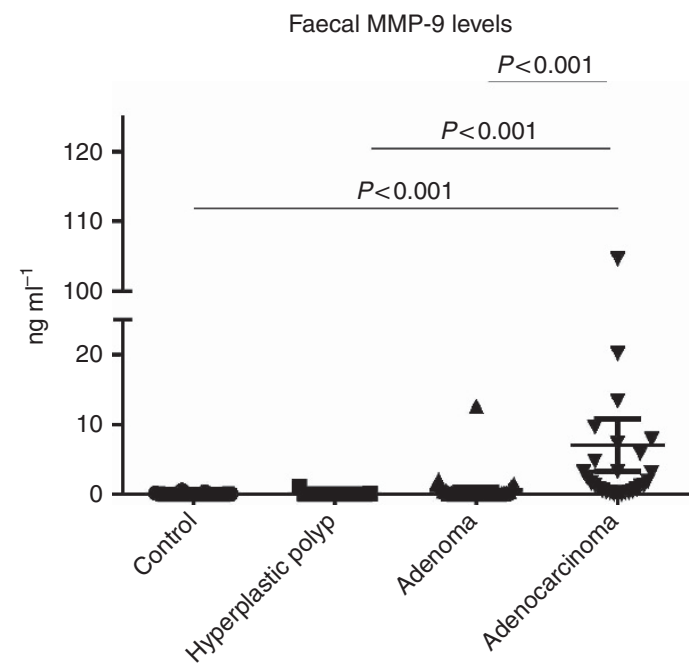

Figure 2. Faecal MMP-9 levels of the different groups (based on colonoscopy and histology). Abbreviation: $\mathrm{CRC}=$ colorectal carcinoma. had slightly, but not significantly elevated faecal MMP-9 levels (mean: $0.656 \pm 0.396 \mathrm{ng} \mathrm{ml}^{-1}$ ). Nevertheless, patients with CRC had a significantly increased mean of faecal MMP-9 levels (mean: $7.028 \pm 3.722 \mathrm{ng} \mathrm{ml}^{-1}$ ). To distinguish CRC patients from controls, the cutoff level was determined at $0.23 \mathrm{ng} \mathrm{ml}^{-1}$ (AUC $=0.913$; 95\% CI: 0.833-0.994; $P<0.001$ ). This cutoff level was associated with a sensitivity of $89.3 \%$ and a specificity of $91.2 \%$ (Figure 3). Patients with hyperplastic polyps (AUC $=0.526 ; 95 \%$ CI: $0.340-0.711 ; P=0.779)$ were not distinguishable from control individuals by ROC analysis. Among adenomas, 22 out of 32 were at increased risk of malignancy, with a mean faecal MMP-9 level significantly elevated compared with low-risk adenomas (mean of high-risk adenomas: $0.936 \pm 0.570$ vs mean of low-risk adenomas: $0.040 \pm 0.017 ; P<0.05$; Figure 4 ). Low-risk adenomas could not be distinguished from controls by faecal MMP-9 levels $(\mathrm{AUC}=0.456$; 95\% CI: $0.236-0.676 ; \quad P=0.706)$ When ROC analysis was performed with an aim to select high-risk adenomas and CRC from controls, the cutoff level was determined at $0.085 \mathrm{ng} \mathrm{ml}^{-1}$. In this case, sensitivity was $76 \%$ and specificity was $85.3 \%$ (AUC $=0.806 ; 95 \%$ CI: $0.710-0.902 ; P<0.001)$. With this lower cutoff level, $59 \%$ of high-risk adenomas were identified. By ROC analysis of high-risk adenomas $v s$ controls, the cutoff level would be similarly $0.085 \mathrm{ng} \mathrm{ml}^{-1}$ (AUC $=0.670 ; 95 \%$ CI: $0.507-0.833$; $P=0.033)$.

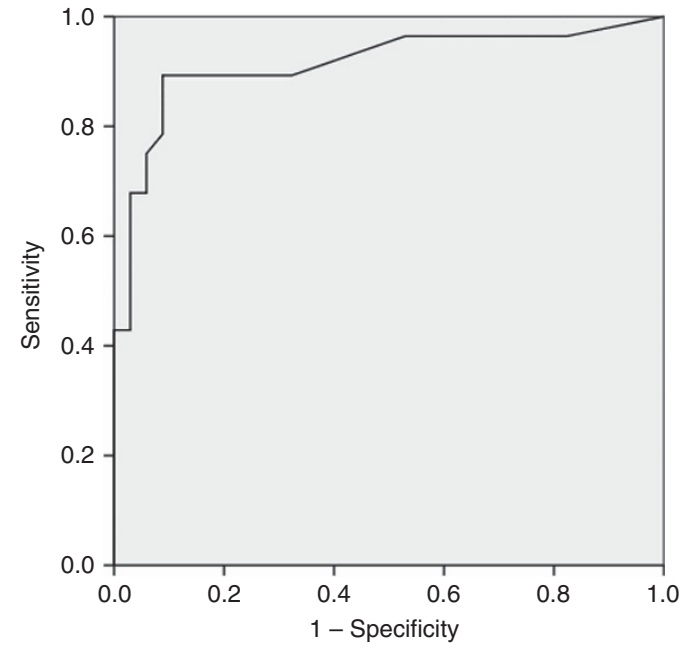

Figure 3. ROC curve of CRC vs controls.

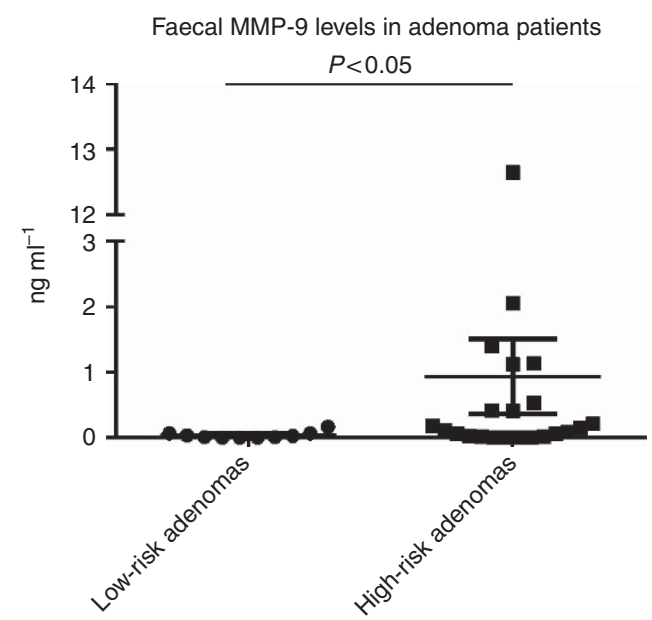

Figure 4. Faecal MMP-9 levels in adenomas. 
A

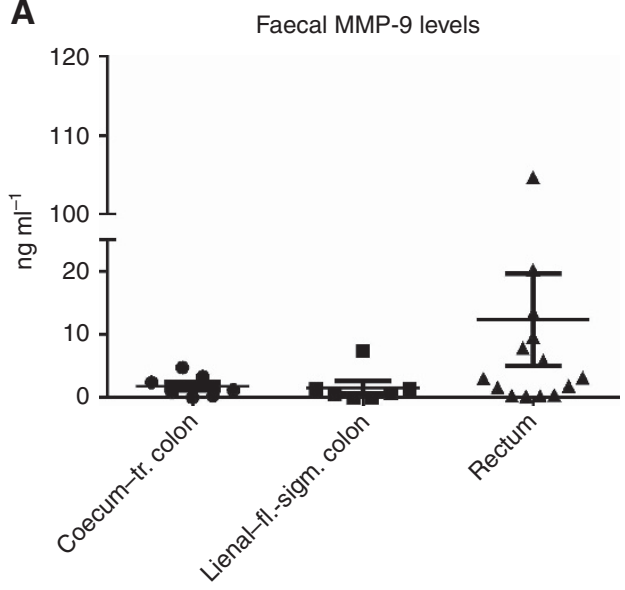

Localisation of adenocarcinoma

B

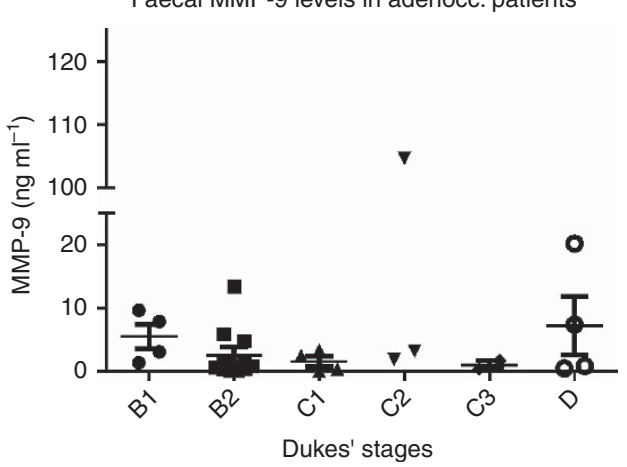

Figure 5. Faecal MMP-9 levels in CRC patients. Values according to (A) localisation and (B) Dukes' stages of CRC.

Faecal MMP-9 levels according to CRC localisation and stage. The number of patients regarding the site of the CRC was as follows: rectum: 14 , sigmoid colon: 6 , lineal flexure: 1 , transverse colon: 2, hepatic flexure: 2 , ascending colon: 1 , and coecum: 2 patients. If faecal MMP-9 levels are analysed according to the site of the CRC, no significant difference was observed $(P=0.146)$, although a trend was seen towards higher levels in case of a rectal tumour (mean: $12.33 \pm 7.274 \mathrm{ng} \mathrm{ml}^{-1}$ ) compared with left-sided colon (lineal flexure-sigmoid colon; mean: $1.619 \pm 0.978 \mathrm{ng} \mathrm{ml}^{-1}$ ) and transverse-right-sided colon (from coecum to transverse colon; mean: $1.830 \pm 0.656 \mathrm{ng} \mathrm{ml}^{-1}$; Figure $5 \mathrm{~A}$ ). According to Dukes' classification, no significant difference could be seen between the groups (Figure 5B; $P=0.236$ ).

\section{DISCUSSION}

To the best of our knowledge, we report for the first time that MMP-9 levels are elevated in faecal specimens from CRC patients compared with patients without polypoid lesions. Furthermore, faecal MMP-9 can be used to distinguish CRC patients from patients with no polypoid lesions, with a high sensitivity and specificity. Additionally, with a lower cutoff level, faecal MMP-9 is able to identify nearly $60 \%$ of patients with a high-risk adenoma.

MMP-9 is a gelatinase capable of the degradation of the extracellular matrix, which is believed to have a role in the progression and metastasis formation of many tumours, including colon cancer (Yang et al, 2014). Apart from colon cancer cells, tumour-infiltrating neutrophils are also an important source of MMP-9, as a major angiogenesis-inducing factor (Hyuga et al,
1994; Deryugina et al, 2014). Serum MMP-9 was suggested as an early noninvasive biomarker for breast cancer (Provatopoulou et al, 2009) and a prognostic marker for lung cancer (Ylisirnio et al, 2000). Urinary MMPs were found elevated not only in bladder, renal and prostate cancers but also in anatomically distant tumours, including breast, brain, and hematological malignancies (Moses et al, 1998; Chan et al, 2004; Fernandez et al, 2005; Smith et al, 2008). Recently, significant efforts have been made to explore the potential role of MMP-9 as a biomarker in CRC. In the serum, it has been suggested as a biomarker of CRC in symptomatic patients (Hurst et al, 2007), and its levels correlated with the Dukes' stage (Mroczko et al, 2010). Furthermore, it has been recently shown that serum levels of neutrophil gelatinaseassociated lipocalin in complex with MMP-9 are elevated in CRC patients, but it was found unsuitable as a diagnostic marker, as its discriminative power was very poor (Duvillard et al, 2014). We have found the discriminative power of faecal MMP-9 levels in CRC patients sufficient, which can be explained by the fact that faecal MMP-9 could be more informative for gastrointestinal malignancies than urine or serum. Our method does not require a preceding diet, and one faecal sample gave a sufficient sensitivity for discrimination. Its analysis is automated, and the cut of concentration is adjustable. In terms of these characteristics, faecal MMP-9 measurement, similarly to FIT, is more convenient for clinical use than gFOBT. Furthermore, faecal MMP-9 measurement provides a higher sensitivity and specificity for CRC than gFOBT and than many types of FIT.

In the past decade, numerous faecal molecular markers have been described, which are based on the detection of genetic mutations and epigenetic alterations, linked to the carcinogenetic process (for a review, see Kanthan et al, 2012). Genetic markers are claimed to be more sensitive than FOBTs (Kanthan et al, 2012). However, one disadvantage of these markers is that they are not present in every colorectal carcinoma. To overcome this problem, genetic markers are often combined in a panel, but this may lead to a decrease in specificity (Kanthan et al, 2012). Furthermore, an important limitation of these methods is their high costs. Current evidence is not sufficient to accept the routine use of faecal genetic markers, and the European Guidelines for quality assurance have stated that they are currently unsuitable for CRC screening (Halloran et al, 2012). Similarly to molecular tests, our method also has the advantage over FOBTs in that it detects a marker originating directly from cancer, which, contrary to bleeding, is supposed to be permanent. However, the costs of faecal MMP-9 measurement are far beyond those of molecular tests, as they are $<10$ USD per sample, which is comparable to the costs of FOBT. This method is markedly cheaper than faecal DNA tests, which cost around 350 USD per sample (Lansdorp-Vogelaar et al, 2010).

The process of transformation from normal epithelium to cancer takes 10-15 years in most cases, leaving a possibility to remove precancerous lesions before they turn to definitive cancer (Binefa et al, 2014). Therefore, a reliable marker detecting high-risk adenomas is of high importance. The sensitivity of FIT for advanced adenomas is only around 20-33\% (Morikawa et al, 2005; Haug et al, 2010). DNA tests are characterised by a much higher sensitivity, for example, K-ras mutations are detected in the faeces of around $50 \%$ of individuals with adenomas $>1 \mathrm{~cm}$ (Mak et al, 2004), p53 mutations in up to $64 \%$ of severely dysplastic polyps (Tagore et al, 2004) and 4-26\% of adenomas (Mak et al, 2004). Methods based on the detection of epigenetic changes appear to have a wide range of sensitivity, for example, the methylation of SFRP2 can be detected in the faeces of $52.4 \%$ of individuals with adenomas (Huang et al, 2007), while the methylation of methylguanine DNA methyltransferase can be found in $36 \%$ of the faeces of adenoma patients but that of the human mut I homolog-1 in only $11 \%$ (Baek et al, 2009). Faecal tumour M2 pyruvate kinase, a promising marker in CRC screening, showed a 
sensitivity of $22 \%$ for advanced adenomas (Haug et al, 2008). Serum MMP-9 levels were elevated not only in cancer but also in colorectal adenoma patients compared with healthy subjects (Mroczko et al, 2010). Similarly, MMP-9 expression was found increased not only in tissue samples from CRC but also in those from highly dysplastic adenomatous polyps compared with hyperplastic polyps or adenomas with low dysplasia (Odabasi et al, 2014). Furthermore, MMP-9 protein expression has been elevated in adenomas with high grade dysplasia compared with other adenomas or normal colonic tissue (Herszenyi et al, 2008). Our results have shown that faecal MMP-9 levels from high-risk adenoma patients are significantly elevated compared with patients with low-risk adenomas. However, with a lower cutoff level, our marker identified 59\% of high-risk adenoma patients as positive. This result is definitely higher than the usual sensitivity of FOBTs and comparable to that of molecular tests but without the high costs of the latter.

We did not find any significant difference between the faecal MMP-9 levels of the groups with different tumour location. Nevertheless, a tendency could be seen towards the highest faecal MMP-9 levels in rectal adenocarcinoma patients. This is probably not related to the nature of rectal tumours but could be explained with a less time for degradation of the marker in the intestinal lumen, similarly to that seen by FIT (Morikawa et al, 2005).

Our study has some limitations. As our department is a specific colorectal center, our patients were a selected population not representing the general population. Patients were on a low-fibre diet at the time of faecal sample collection, as a preparation for the upcoming colonoscopy. Data on the time of the day at faecal sample production was not collected in our study. Although the percentage of cancer and adenoma found on colonoscopy was relatively high, the number of patients in the different subgroups (i.e., tumour localisation, Dukes' stage) may be insufficient for the detection of minor differences. Moreover, MMP-9 is also elevated in gut inflammation, which may limit its use in case of, for example, IBD. We hypothesise that, in a more general population, elevated faecal MMP-9 levels in some cases could be related to inflammation or other cancers in the gastrointestinal tract. However, even in this case, the method would be a good predictor of the presence of a nonfunctional gastrointestinal disorder, based on our previous results showing that faecal MMP-9 measurement can well discriminate IBS-D patients and healthy subjects from UC patients. A further limitation is that, owing to the different cutoff levels calculated on the same sample groups, the performance of the method may be overestimated. A further study with a larger and more general patient population could help us to clarify whether diet, time of the day at sampling, tumours of the upper GI tract and so on can affect faecal MMP-9 levels and could also serve to verify the performance of our method. A comparison with FIT on the same samples could be useful. Moreover, a larger study on a general cohort could also provide more information if this method is valuable for CRC screening.

Nonetheless, our study was suitable to identify a new marker, faecal MMP-9, capable of discrimination of CRC from polyp-free controls. Faecal MMP-9 is characterised by many advantages of FIT and also of the molecular markers, without high costs. Further studies on a larger and more general population are needed to confirm whether this promising method is suitable for CRC screening as a complementary assay.

\section{ACKNOWLEDGEMENTS}

This work was supported by the institutional grants from TÁMOP (TAMOP-4.2.2.A-11/1/KONV-2012-0035, TAMOP-4.2.2-A-11/1/ KONV-2012-0052 TAMOP-4.2.2.A-11/1/KONV-2012-0073).
CONFLICT OF INTEREST

The authors declare no conflict of interest.

\section{REFERENCES}

Annahazi A, Molnar T, Farkas K, Rosztoczy A, Izbeki F, Gecse K, Inczefi O, Nagy F, Foldesi I, Szucs M, Dabek M, Ferrier L, Theodorou V, Bueno L, Wittmann T, Roka R (2013) Fecal MMP-9: a new noninvasive differential diagnostic and activity marker in ulcerative colitis. Inflamm Bowel Dis 19(2): 316-320.

Araujo Jr RF, Lira GA, Vilaca JA, Guedes HG, Leitao MC, Lucena HF, Ramos CC (2015) Prognostic and diagnostic implications of MMP-2, MMP-9, and VEGF-alpha expressions in colorectal cancer. Pathol Res Pract 211(1): 71-77.

Baek YH, Chang E, Kim YJ, Kim BK, Sohn JH, Park DI (2009) Stool methylation-specific polymerase chain reaction assay for the detection of colorectal neoplasia in Korean patients. Dis Colon Rectum 52(8): 1452-1459; discussion 1459-63.

Baker GS, Kramer BS (2007) Peirce, Youden, and receiver operating characteristic curves. Am Stat 61(4): 343-346.

Binefa G, Rodriguez-Moranta F, Teule A, Medina-Hayas M (2014) Colorectal cancer: from prevention to personalized medicine. World $J$ Gastroenterol 20(22): 6786-6808.

Chan LW, Moses MA, Goley E, Sproull M, Muanza T, Coleman CN, Figg WD, Albert PS, Menard C, Camphausen K (2004) Urinary VEGF and MMP levels as predictive markers of 1-year progression-free survival in cancer patients treated with radiation therapy: a longitudinal study of protein kinetics throughout tumor progression and therapy. J Clin Oncol 22(3): 499-506.

Deryugina EI, Zajac E, Juncker-Jensen A, Kupriyanova TA, Welter L, Quigley JP (2014) Tissue-infiltrating neutrophils constitute the major in vivo source of angiogenesis-inducing MMP-9 in the tumor microenvironment. Neoplasia 16(10): 771-788.

Duvillard L, Ortega-Deballon P, Bourredjem A, Scherrer ML, Mantion G, Delhorme JB, Deguelte-Lardiere S, Petit JM, Bonithon-Kopp C (2014) A case-control study of pre-operative levels of serum neutrophil gelatinaseassociated lipocalin and other potential inflammatory markers in colorectal cancer. BMC Cancer 14: 912.

Farkas K, Balint A, Bor R, Foldesi I, Szucs M, Nagy F, Szepes Z, Annahazi A, Roka R, Molnar T (2014) Faecal matrix metalloprotease-9 is a more sensitive marker for diagnosing pouchitis than faecal calprotectin: results from a pilot study. Expert Rev Gastroenterol Hepatol 9(3): 387-392.

Ferlay J, Steliarova-Foucher E, Lortet-Tieulent J, Rosso S, Coebergh JW, Comber H, Forman D, Bray F (2013) Cancer incidence and mortality patterns in Europe: estimates for 40 countries in 2012. Eur J Cancer 49(6): 1374-1403.

Fernandez CA, Yan L, Louis G, Yang J, Kutok JL, Moses MA (2005) The matrix metalloproteinase-9/neutrophil gelatinase-associated lipocalin complex plays a role in breast tumor growth and is present in the urine of breast cancer patients. Clin Cancer Res 11(15): 5390-5395.

Halloran SP, Launoy G, Zappa M (2012) European guidelines for quality assurance in colorectal cancer screening and diagnosis. First EditionFaecal occult blood testing. Endoscopy 44(Suppl 3): SE65-SE87.

Haug U, Hundt S, Brenner H (2008) Sensitivity and specificity of faecal tumour M2 pyruvate kinase for detection of colorectal adenomas in a large screening study. Br J Cancer 99(1): 133-135.

Haug U, Hundt S, Brenner H (2010) Quantitative immunochemical fecal occult blood testing for colorectal adenoma detection: evaluation in the target population of screening and comparison with qualitative tests. Am J Gastroenterol 105(3): 682-690.

Herszenyi L, Sipos F, Galamb O, Solymosi N, Hritz I, Miheller P, Berczi L, Molnar B, Tulassay Z (2008) Matrix metalloproteinase-9 expression in the normal mucosa-adenoma-dysplasia-adenocarcinoma sequence of the colon. Pathol Oncol Res 14(1): 31-37.

Huang Z, Li L, Wang J (2007) Hypermethylation of SFRP2 as a potential marker for stool-based detection of colorectal cancer and precancerous lesions. Dig Dis Sci 52(9): 2287-2291.

Hurst NG, Stocken DD, Wilson S, Keh C, Wakelam MJ, Ismail T (2007) Elevated serum matrix metalloproteinase 9 (MMP-9) concentration 
predicts the presence of colorectal neoplasia in symptomatic patients. $\mathrm{Br} J$ Cancer 97(7): 971-977.

Hyuga S, Nishikawa Y, Sakata K, Tanaka H, Yamagata S, Sugita K, Saga S, Matsuyama M, Shimizu S (1994) Autocrine factor enhancing the secretion of $M(r)$ 95,000 gelatinase (matrix metalloproteinase 9) in serum-free medium conditioned with murine metastatic colon carcinoma cells. Cancer Res 54(13): 3611-3616.

Kanthan R, Senger JL, Kanthan SC (2012) Fecal molecular markers for colorectal cancer screening. Gastroenterol Res Pract 2012: 184343.

Konrad G (2010) Dietary interventions for fecal occult blood test screening: systematic review of the literature. Can Fam Physician 56(3): 229-238.

Lansdorp-Vogelaar I, Kuntz KM, Knudsen AB, Wilschut JA, Zauber AG, van Ballegooijen M (2010) Stool DNA testing to screen for colorectal cancer in the Medicare population: a cost-effectiveness analysis. Ann Intern Med 153(6): 368-377.

Mak T, Lalloo F, Evans DG, Hill J (2004) Molecular stool screening for colorectal cancer. Br J Surg 91(7): 790-800.

Morikawa T, Kato J, Yamaji Y, Wada R, Mitsushima T, Shiratori Y (2005) A comparison of the immunochemical fecal occult blood test and total colonoscopy in the asymptomatic population. Gastroenterology 129(2): $422-428$.

Moses MA, Wiederschain D, Loughlin KR, Zurakowski D, Lamb CC, Freeman MR (1998) Increased incidence of matrix metalloproteinases in urine of cancer patients. Cancer Res 58(7): 1395-1399.

Mroczko B, Groblewska M, Okulczyk B, Kedra B, Szmitkowski M (2010) The diagnostic value of matrix metalloproteinase 9 (MMP-9) and tissue inhibitor of matrix metalloproteinases 1 (TIMP-1) determination in the sera of colorectal adenoma and cancer patients. Int J Colorectal Dis 25(10): 1177-1184.

Odabasi M, Yesil A, Ozkara S, Paker N, Ozkan S, Eris C, Yildiz MK, Abuoglu HH, Gunay E, Tekesin K (2014) Role of human neutrophil gelatinase associated lipocalin (NGAL) and Matrix Metalloproteinase-9 (MMP-9) overexpression in neoplastic colon polyps. Int J Clin Exp Med 7(9): 2804-2811.
Provatopoulou X, Gounaris A, Kalogera E, Zagouri F, Flessas I, Goussetis E, Nonni A, Papassotiriou I, Zografos G (2009) Circulating levels of matrix metalloproteinase-9 (MMP-9), neutrophil gelatinase-associated lipocalin (NGAL) and their complex MMP-9/NGAL in breast cancer disease. BMC Cancer 9: 390.

Siegel R, Naishadham D, Jemal A (2012) Cancer statistics, 2012. CA Cancer J Clin 62(1): 10-29.

Smith ER, Zurakowski D, Saad A, Scott RM, Moses MA (2008) Urinary biomarkers predict brain tumor presence and response to therapy. Clin Cancer Res 14(8): 2378-2386.

Sung JJ, Lau JY, Young GP, Sano Y, Chiu HM, Byeon JS, Yeoh KG, Goh KL, Sollano J, Rerknimitr R, Matsuda T, Wu KC, Ng S, Leung SY, Makharia G, Chong VH, Ho KY, Brooks D, Lieberman DA, Chan FK (2008) Asia Pacific consensus recommendations for colorectal cancer screening. Gut 57(8): 1166-1176.

Tagore KS, Levin TR, Lawson MJ (2004) The evolution to stool DNA testing for colorectal cancer. Aliment Pharmacol Ther 19(12): 1225-1233.

Unsal D, Akyurek N, Uner A, Erpolat OP, Han U, Akmansu M, Mentes BB, Dursun A (2008) Gelatinase B expression as a prognostic factor in patients with stage II/III rectal carcinoma treated by postoperative adjuvant therapy. Am J Clin Oncol 31(1): 55-63.

Yang B, Tang F, Zhang B, Zhao Y, Feng J, Rao Z (2014) Matrix metalloproteinase- 9 overexpression is closely related to poor prognosis in patients with colon cancer. World J Surg Oncol 12: 24.

Ylisirnio S, Hoyhtya M, Turpeenniemi-Hujanen T (2000) Serum matrix metalloproteinases $-2,-9$ and tissue inhibitors of metalloproteinases $-1,-2$ in lung cancer-TIMP-1 as a prognostic marker. Anticancer Res 20(2B): 1311-1316.

This work is published under the standard license to publish agreement. After 12 months the work will become freely available and the license terms will switch to a Creative Commons AttributionNonCommercial-Share Alike 4.0 Unported License. 